Hydrodynamically Coupled Brownian Dynamics simulations for flow of non-Newtonian fluids

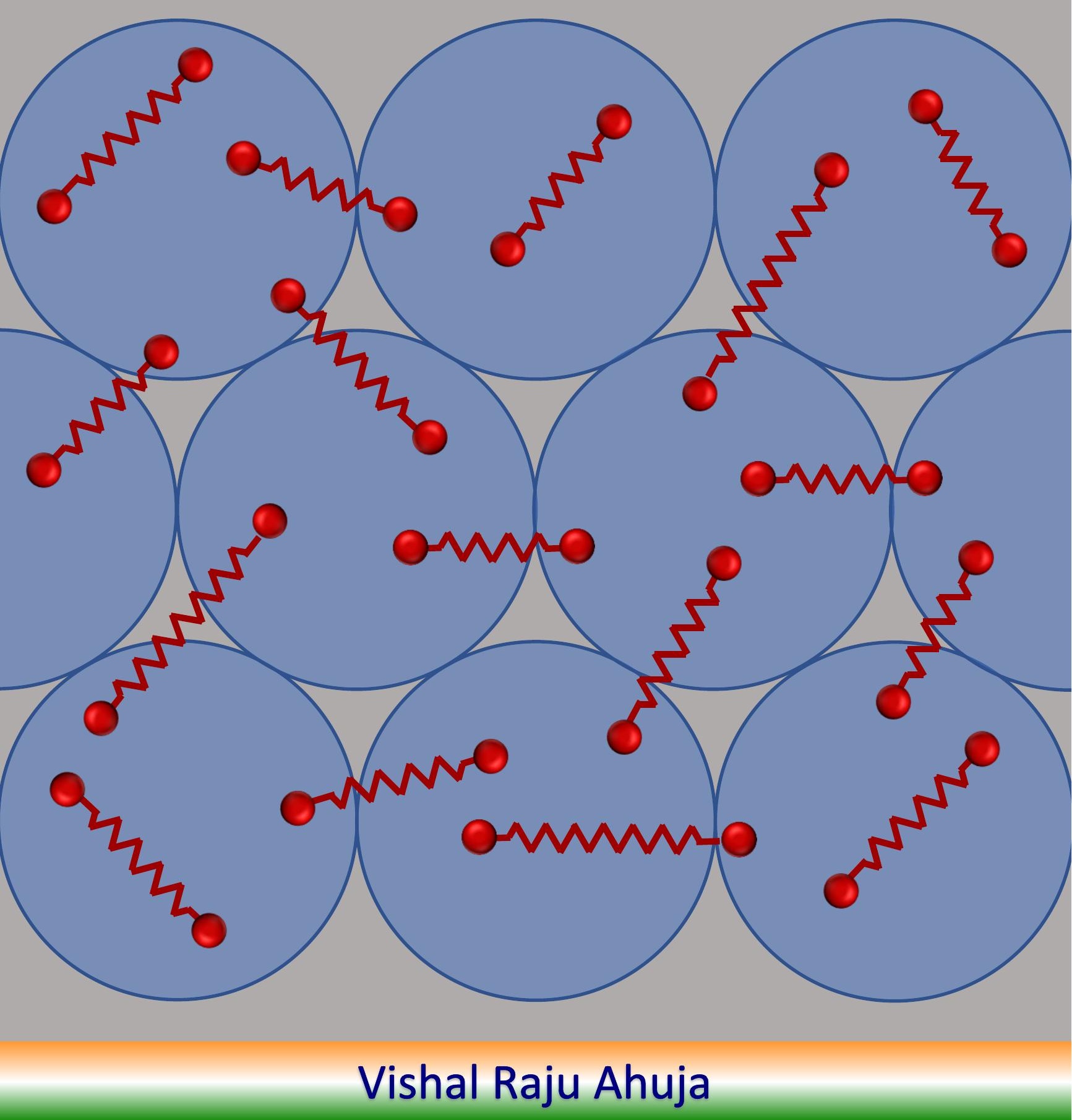




\title{
HYDRODYNAMICALLY COUPLED BROWNIAN DYNAMICS SIMULATIONS FOR FLOW OF NON-NEWTONIAN FLUIDS
}

\section{DISSERTATION}

\author{
to obtain \\ the degree of doctor at the University of Twente, \\ on the authority of the rector magnificus, \\ Prof. dr. T. T. M. Palstra, \\ on account of the decision of the Doctorate Board, \\ to be publicly defended \\ on Friday, June $15^{\text {th }}, 2018$ at 14:45 hours
}

by

\section{Vishal Raju Ahuja}

born on November $5^{\text {th }}, 1989$ in Mumbai, India. 
This dissertation has been approved by:

Supervisor: Prof. dr. W. J. Briels University of Twente

Supervisor: Prof. dr. ir. J. van der Gucht Wageningen University

(C) 2018: Vishal Raju Ahuja, Enschede, The Netherlands.

All rights reserved. No part of this thesis may be reproduced or transmitted in any form, by any means, electronic or mechanical, without prior written permission of the author.

Cover Art: A schematic diagram of the novel computational technique presented in this thesis called Hydrodynamically Coupled Brownian Dynamics (HCBD).

Typeset in $\mathrm{LT}_{\mathrm{E}} \mathrm{X}$. Printed by IPSKAMP.

ISBN : $\quad$ 978-90-365-4562-4

DOI : $\quad 10.3990 / 1.9789036545624$

URL : https://doi.org/10.3990/1.9789036545624 


\section{Graduation Committee:}

Chairman: Prof. dr. ir. J. W. M. Hilgenkamp

Supervisor: Prof. dr. W. J. Briels

Supervisor: Prof. dr. ir. J. van der Gucht

Members: Prof. dr. V. G. Mavrantzas

Prof. dr. J. K. G. Dhont

Prof. dr. ir. J. M. V. A. Koelman

Dr. ir. J. T. Padding

Prof. dr. F. G. Mugele

Prof. dr. ir. S. J. G. Lemay
University of Twente

University of Twente

Wageningen University

University of Patras

Forschungszentrum Jülich

Eindhoven University of Technology

Delft University of Technology

University of Twente

University of Twente

The research presented in this thesis was conducted in the Computational Chemical Physics (CCP) group, MESA+ Institute of Nanotechnology at the University of Twente in collaboration with the Physical Chemistry and Soft Matter group of Wageningen University. This work is part of the Industrial Partnership Programme (IPP) Computational sciences for energy research of the Foundation for Fundamental Research on Matter (FOM), which is part of the Netherlands Organisation for Scientific Research (NWO). This research programme is co-financed by Shell Global Solutions International B.V. 

काल नहीं है बीतता,

बीत रहे हम लोग।

भोग नहीं हम भोगते,

भोगे हमको भोग।

- गोपालदास सक्षेना 'नीरज'

Translation*:

'Tis not times that pass,

'tis we, who through times pass.

'Tis not we, who consume the things, 'tis the things that consume us.

- Gopaldas Saxena 'Neeraj'

*Disclaimer: This is a poetic translation attempted by me (based on my personal interpretation of the original lines by Neeraj in Hindi)

A few lines about my favourite poet - Neeraj:

Awarded the prestigious Indian Padma awards - Padma Shri in 1991 and Padma Bhushan in 2007, Neeraj is a highly regarded poet and writer of Hindi literature. He is now 93 years old and still enthrals the audiences at Kavi Sammelans (public gatherings of poets) with his deep sagelike voice and poetic style. 



\section{Contents}

1 Introduction 1

1.1 Classification: Newtonian vs Non-Newtonian fluids . . . . . . . . . . 1

1.2 Primary application : Enhanced Oil Recovery . . . . . . . . . . 3

1.3 Modeling Approach: Particle-based simulations . . . . . . . . . . . . 5

1.4 Thesis outline . . . . . . . . . . . . . . . . 7

Bibliography ........................... 12

2 Two-way coupling of polymers with implicit solvent 13

2.1 Introduction . . . . . . . . . . . . . . . . . . . . . 14

2.2 Model development . . . . . . . . . . . . . . . . . 18

2.2.1 Update of positions . . . . . . . . . . . . 18

2.2.2 Update of velocities . . . . . . . . . . . . . . 19

2.2.3 Interaction with solid walls . . . . . . . . . . . 22

2.3 Test System and Parameters . . . . . . . . . . . . . . 23

2.3.1 Test system . . . . . . . . . . . . . 23

2.3.2 Definitions of weight functions and the characteristic time constant ...................... 24

2.3.3 Parameter Values . . . . . . . . . . . . . . . . 25

2.4 Results and Discussion . . . . . . . . . . . . . . . 25

2.4.1 Bulk flow simulations in quiescent state . . . . . . . . . 25

2.4.2 Flow simulations with solid interfaces . . . . . . . . . 33

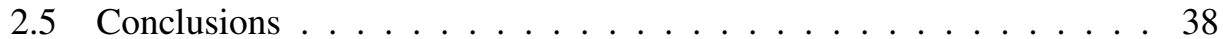

2.6 Appendix: Mean Squared Displacement of non-interacting particles . 39

Bibliography ......................... 44

3 Two-way coupling of polymers with explicit solvent 45

3.1 Introduction . . . . . . . . . . . . . . . . . . 46

3.2 Model development . . . . . . . . . . . . . . . . . . . 48

3.2.1 Equation of motion for the polymer blobs . . . . . . . . . 48 
3.2.2 Equation of motion for the fluid blobs . . . . . . . . . . 50

3.3 Test System and Parameters . . . . . . . . . . . . . . 54

3.3.1 The conservative potential ............ 54

3.3.2 The transient potential ............... 55

3.3.3 The equation of state .............. 55

3.3.4 Definition of weight functions and system parameters . . . . 56

3.4 Results and Discussion . . . . . . . . . . . . . . . . . 59

3.4.1 Pure fluid simulations . . . . . . . . . . . . 59

3.4.2 Polymer solution simulations . . . . . . . . . . 63

3.5 Conclusions ......................... 67

3.6 Appendix ........................ 70

3.6.1 Proof of local momentum conservation for the interaction between polymer and fluid blobs . . . . . . . . . . 70

3.6.2 Derivation of stochastic update for the fluid velocities . . . . . 71

Bibliography ...................... 77

4 Shear Banding and Shear Rolls $\quad 79$

4.1 Introduction . . . . . . . . . . . . . . . 80

4.2 Model ......................... 82

4.2.1 Equation of motion for the polymer blobs . . . . . . . . 82

4.2.2 Equation of motion for the fluid blobs . . . . . . . . 83

4.3 Potentials and System Parameters . . . . . . . . . . . . 85

4.3.1 Potentials.................. 85

4.3.2 Weight functions and resolution ........... 87

4.3.3 System parameters ................ 89

4.4 Results and Discussion . . . . . . . . . . . . . . . . . 89

4.4.1 Shear Banding . . . . . . . . . . . . 90

4.4 .2 Shear Rolls . . . . . . . . . . . . . . . . . . 96

4.5 Conclusion and scope for further research . . . . . . . . . . . 100

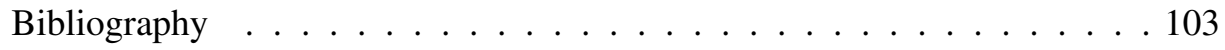


5 Flow through porous media $\quad 105$

5.1 Introduction . . . . . . . . . . . . . . 106

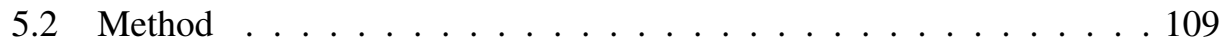

5.2.1 Equation of motion for the polymer blobs . . . . . . . . 109

5.2.2 Equation of motion for the fluid blobs . . . . . . . . 111

5.3 Test System and Parameters . . . . . . . . . . . . . . . . . . 112

5.3.1 The conservative potential . . . . . . . . . 112

5.3.2 The transient potential ................ 113

5.3.3 The FENE Potential . . . . . . . . . . . . . . . . . . . . 114

5.3.4 The equation of state . . . . . . . . . . . . . 114

5.3.5 Definition of weight functions and system parameters . . . . . 114

5.3.6 Interaction of the fluid with the solid . . . . . . . . . . 117

5.4 Results and Discussion . . . . . . . . . . . . . . . . . 118

5.4.1 Flow through cylindrical porous media . . . . . . . . . 118

5.4.2 Flow through cuboidal porous media . . . . . . . . . 127

5.5 Conclusion and scope for further research . . . . . . . . . . 133

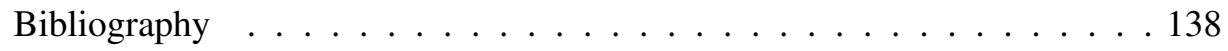

$\begin{array}{llr}6 & \text { Summary } & 139\end{array}$

$\begin{array}{lll}7 & \text { Samenvatting } & 143\end{array}$

8 Scientific Output $\quad 147$

8.1 Publications . . . . . . . . . . . . . . . . . 147

8.2 Talks . . . . . . . . . . . . . . . . . . 147

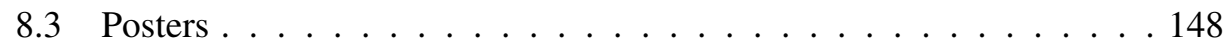

9 Social Impact $\quad 149$

10 Acknowledgments $\quad 153$ 



\section{Introduction}

\subsection{Classification: Newtonian vs Non-Newtonian fluids}

In this thesis, we deal with the flow of non-Newtonian fluids and our focus is particularly on polymer solutions, which are known for exhibiting non-Newtonian flow behavior. Before delving further into the complex flow of non-Newtonian fluids such as polymer solutions, we briefly explain at the outset which fluids are classified as Newtonian / non-Newtonian and what does it really mean from a macroscopic flow perspective.

Fluids consisting of small molecules, say with a molecular weight of upto a 1000 Daltons, are classified as Newtonian fluids because they exhibit a linear relationship between the stress tensor $\tau$ and the rate-of-deformation tensor $\Gamma$, which is known as Newton's law of viscosity.[1] Since we only deal with liquids in this thesis, we only show the incompressible version of all the equations. Thus, for incompressible liquids, Newton's law of viscosity essentially reduces to eq.(1.1).

$$
\tau=-2 \eta \Gamma,
$$

where $\eta$ is the viscosity of the fluid and the rate-of-deformation tensor $\Gamma$ is defined as $\frac{1}{2} \boldsymbol{\nabla} \mathbf{v}+\frac{1}{2} \boldsymbol{\nabla} \mathbf{v}^{T}$, wherein $\boldsymbol{\nabla} \mathbf{v}$ is the gradient of the velocity $\mathbf{v}$ of the fluid and the next term is the transpose of the first term. On the other hand, when large molecules such as polymers are present in the fluid, this linear relationship between the stress tensor and the rate-of-deformation tensor does not hold anymore, and hence they are classified as non-Newtonian fluids. But what does this really mean from a macroscopic flow perspective? This difference results in non-Newtonian fluids having a qualitatively different flow behavior from simple Newtonian fluids. There are many examples of the differences in their flow behaviors such as the characteristic flattening of the velocity profile of shear thinning polymer solutions as opposed to the characteristic parabolic velocity profile for a Newtonian fluid flowing through a tube with a circular cross-section (Poiseuille flow) or between parallel plates (plane Poiseuille flow), the tubeless siphon phenomenon observed for polymeric liquids that is not observed for Newtonian fluids, etc. We do not describe these phenomena here in full 
detail as there are plenty of such examples and they have been described very well in the literature.[1, 2]

Having explained the difference between Newtonian and non-Newtonian fluids and its consequence on macroscopic flow behavior, we now briefly describe the fundamental equations for describing the flow of these fluids. The flow of incompressible liquids can be very satisfactorily described using the well-known Navier-Stokes equations for incompressible fluid flow, shown in eq.(1.2) in tensor notation, which can easily be arrived at by using the principle of momentum conservation.[1]

$$
\rho \frac{D \mathbf{v}}{\mathrm{d} t}=-\nabla P-\nabla \cdot \tau+\rho \mathbf{g}
$$

where $\rho$ is the mass density of the fluid, $D \mathbf{v} / \mathrm{d} t$ is the material derivative of the velocity of the fluid, $\nabla P$ is the pressure gradient, $\nabla . \tau$ is the divergence of the stress tensor and $\mathbf{g}$ represents accelerations due to body forces such as gravity etc.

The second term on the right hand side of eq.(1.2), i.e. the divergence of the stress tensor requires an expression for the stress tensor for closure of the set of equations. For Newtonian fluids, the stress tensor can be readily calculated by using Newton's law of viscosity shown in eq.(1.1), which is essentially a simple linear constitutive equation for the dependence of the stress tensor on the rate-of-deformation tensor. This linear constitutive equation for the Newtonian fluids leads to simple solutions that can be analytically calculated for several flow situations - even for flow through fairly complex geometries.[1] However, the same cannot be said for non-Newtonian fluids because even if various approximate complicated constitutive equations can be constructed for these fluids in principle,[2] it is certainly not possible to solve them analytically, particularly for flow through complex geometries.

Hence, advanced simulation methodologies are required to model the complex flow of non-Newtonian fluids such as polymer solutions. The development and application of such theoretical models to simulate flow of these complex fluids is the primary focus of this thesis. Basic details about the fundamental equations of the computational model that we have developed for simulating flow of these complex fluids such as polymer solutions are provided in subsection 1.4 of this chapter. 


\subsection{Primary application : Enhanced Oil Recovery}

Studying flow of non-Newtonian fluids such as polymer solutions has wide-ranging applications such as polymer extrusion, food-processing, enhanced oil recovery using polymer flooding etc. Of these applications, we focus our attention on enhanced oil recovery using polymer flooding as that is the primary motivation for the funding of this project. The production of crude oil from oil-reservoirs typically involves one or more steps of a multi-step process comprising of primary recovery, secondary recovery and enhanced oil recovery, each of which adds to the total amount of oil recovered from the reservoir.[3] The amount of oil produced by these three stages varies considerably for different types of hydrocarbons - whether they are light oils, heavy oils or tar sands and also depends on the internal structure of the reservoir its porosity, heterogeneity, etc. Initial production results from the natural energy of the oil in the reservoir and the rock decompression, which naturally drives the oil out of the production wells leading to a recovery of about 5 to $25 \%$ of the Original Oil In Place (OOIP). Secondary recovery is then employed to increase the yield from the reservoir while maintaining the pressure of the field. It typically involves injecting water into the reservoir through dedicated injection wells and is typically continued till the amount of water in the produced fluid becomes too high. This secondary recovery produces typically about 5 to $30 \%$ OOIP. Thus, even after the primary and secondary recovery, typically more than half of the original oil in the reservoir is still trapped inside the reservoir, which can be recovered by a number of different techniques grouped together as Enhanced Oil Recovery (EOR) techniques.

There are several different techniques classified as EOR techniques, whose main motivation is to recover additional oil by lowering the oil saturation below the residual oil saturation, which is the amount of residual oil retained in the reservoir either due to capillary forces after a waterflood in light oil reservoirs or as a result of the oil being practically immobile due its high viscosity as is the case with heavy oils. The EOR methods are broadly classified as thermal and non-thermal. Thermal EOR methods usually involve the use of steam or sometimes hot water and are typically 
used for recovering heavy oils because heating lowers the viscosity of the heavy oil and thereby increases its recovery. Non-thermal EOR methods are mainly suited for light oils ( $<100 \mathrm{cp}$ ) and may be used for moderately viscous oils ( $<2000 \mathrm{cp})$, which are unsuitable for thermal methods. The main objectives of these methods are lowering the interfacial tension between the oil and the displacing fluid and / or improving the mobility ratio between these two fluids. Miscible flooding is one type of non-thermal EOR method where the displacing fluid is miscible with the reservoir oil either at first contact or after multiple contacts, thereby reducing the interfacial tension to zero. Another type of non-thermal EOR method is chemical flooding, which involves the use of a chemical formulation as a displacing fluid which decreases the mobility ratio between the displacing fluid and the oil and/or decreases the interfacial tension.

Depending on the chemical formulation used as the displacing fluid, chemical flooding may be classified into polymer flooding, surfactant flooding, alkaline flooding, micellar flooding, and alkaline-surfactant-polymer flooding. As the name suggests, surfactant flooding involves the use of aqueous surfactants which lower the interfacial tension between the aqueous driving fluid and the oil. Alkaline flooding involves the use of an aqueous solution of an alkaline chemical which reacts with acidic components of the crude oil to produce surfactants in situ. Polymer flooding involves the use of water soluble polymers such as polyacrylamides, which help in improving the mobility ratio between the aqueous driving fluid and the oil, thereby reducing the permeability contrast and driving the oil out with the driving fluid.[4] It is this application that we keep in mind while developing the methods that we present in this thesis. The flow of polymer solutions, which are non-Newtonian fluids as mentioned in the previous sub-section, through the complex geometry of the oil reservoir can be quite complex. Hence, it is our objective to shed some light on the flow of polymer solutions through porous media, which can help improve the understanding of the flow of polymer solutions through the oil reservoir, thereby improving the volumetric sweep efficiency of the polymer flooding process for enhanced oil recovery. 


\subsection{Modeling Approach: Particle-based simulations}

As mentioned in the first section of this chapter, the flow of non-Newtonian fluids such as polymer solutions is a complex subject, the understanding of which has been constantly improving as more advancements are being made in the domain of experiments as well as simulations. Experiments have surely provided valuable information as visualization of the flows of these complex fluids have helped to solve many industrial problems. However, as the fundamental knowledge of this field has grown, more complicated problems are now being targeted and the experiments are therefore becoming more tedious, giving way to computer simulations.[2] Simulations can be broadly categorized into two categories - Computational Fluid Dynamics (CFD) simulations and Particle-based simulations. CFD simulations approach the problem using a continuum approximation of the polymer solution, meaning that the solution is considered as a continuum fluid with a particular constitutive equation used to describe the relationship between the stress tensor and the rate-of-deformation tensor. The constitutive equation is used to calculate the divergence of the stress tensor in the Navier-Stokes equation, which is then discretized on a Eulerian grid and solved numerically. Particle-based simulations on the other hand represent the polymer solution with particles and assume no constitutive relationship between the stress tensor and the rate-of-deformation tensor but rather let it evolve from molecular interactions. Thus, particle based simulations have the added advantage of being able to relate macroscopic flow phenomena with microscopic molecular interactions based on the structure of the molecules. It is the latter approach, i.e. the particle-based simulation approach that we have used in this thesis to study the flow of non-Newtonian fluids such as polymer solutions.

Among the particle-based simulation techniques, there is again a wide variety of techniques that can be used for simulating polymer solutions, but the one thing that is common amongst all these particle-based simulation techniques is coarse-graining. Since the polymer molecules are orders of magnitude larger in size than the molecules of the solvent (say water) that they are dissolved in, it is practically impossible to 
resolve all the polymer and water molecules down to the atomic level. Hence, coarsegraining is an inherent part of simulating polymer solutions on a large scale. Again, there are several levels of coarse-graining that may be employed with different levels of molecular detail resolved. However, for the application that we intend to use our simulations for, i.e. polymer flooding through micro-channels, it is practically impossible to resolve the polymers to anything more than a couple of particles per polymer molecule. Similarly, the aqueous solvent has to be highly coarse-grained too and thus may be at best resolved to large blobs containing a very large number of water molecules (of the order of $10^{13}$ molecules). Thus, we use highly coarse-grain particle-based simulations in this work keeping the application of polymer flooding in mind.

Consider a polymer solution in a quiescent state, i.e. a state in which the fluid in which the polymers are dissolved is stationary. Even when the background fluid is not flowing, the polymers experience random motion due to thermal fluctuations. This motion leads to a mean-squared-displacement of the polymers that is directly proportional to time. This can be effectively captured by using a Brownian Dynamics algorithm, with special interactions between the coarse-grained polymers to account for the neglected degrees of freedom. Now, consider that the fluid in which the polymers are dissolved is not stationary, but rather flowing through a complex geometry. This is when it gets very tricky because now you need an algorithm that can couple the motion of the polymers with the flow field of the background fluid, which is in turn influenced by the motion of the polymers. Thus, it is not possible for example to calculate the flow field of the background fluid without the polymers and then impose this field onto the motion of the polymers because this would only lead to a one-way coupling, where only the polymers are influenced by the flow field of the background fluid but not vice versa. Hence, there is a need for an algorithm that can calculate the flow of the background fluid on the fly while exchanging momentum with the polymers. Thus, our idea in this thesis is to develop coarse-grain particle based simulations that can calculate the self-developing flow of polymer solutions using a two-way coupling between the polymers and the fluid. 


\subsection{Thesis outline}

We start with a simple model in Chapter 2, where the polymers are represented by their centers-of-mass and the background fluid is assumed to be implicitly present at the positions of the centres-of-mass of the polymer blobs.[5, 6] This makes the coupling easy as the force experienced by the polymers due to the inter-polymer potentials can be easily transmitted to the fluid. At the same time, it is easy to transmit the fluid flow field back to the polymers as the centers-of-mass of the polymers themselves have been used as the node points for the discretization and solution of the fluid flow field. Here, we used a potential that is commonly used for star polymers and hence simulated the flow of model star polymer solutions - both in bulk as well as in the presence of solid interfaces where appropriate boundary conditions of no-slip were applied. Even with this simple model, we did manage to capture the flattening of the Poiseuille flow profile typically observed for shear thinning polymers. Although the simplicity of this model makes it very efficient, nevertheless, this model has its limitations such as not being able to decide the resolution of the fluid independently from the concentration of the polymers and not being able to decide the equation of state for the fluid. These difficulties could only be resolved if two different types of particles were used for the polymers and the fluid. However, in this case, the coupling between the polymers and the fluid becomes tricky as the fluid is no longer implicitly present at the positions of the centres-of-mass of the polymer blobs.

This led to the development of a new technique described in Chapter 3 - Hydrodynamically Coupled Brownian Dynamics (HCBD), [7] in which we indeed used two different types of particles for the polymers and the fluid. For the interaction between the polymers, we used a Brownian Dynamics based technique called Responsive Particle Dynamics (RaPiD), [8, 9] and for calculating the fluid flow field, we used the phenomenological approach of Smoothed Particle Hydrodynamics (SPH).[10, 11] However, the most important part of HCBD is of course the coupling between the microscopic polymer model and the phenomenological fluid model. We have coupled the two in such a way that not only is there no lag between the polymer and the fluid 
but also the momentum is locally conserved, preserving long-range hydrodynamics.

We now briefly present the fundamental equations of motion for polymers and fluid blobs in our HCBD technique, the details of which can be found in the following chapters of the thesis. The equation of motion for a mesoscopic polymer blob $a$ present in a fluid is given by a first order Brownian Dynamics position update shown in eq.(1.3).

$$
\mathrm{d} \mathbf{r}_{a}=\mathbf{v}\left(\mathbf{r}_{a}\right) \mathrm{d} t+\left(\frac{\mathbf{F}_{a}}{\xi_{a}}\right) \mathrm{d} t+k_{B} T \frac{\partial}{\partial \mathbf{r}_{a}}\left(\frac{1}{\xi_{a}}\right) \mathrm{d} t+\mathrm{d} \mathbf{W}_{a}^{R}
$$

where $\mathbf{v}\left(\mathbf{r}_{a}\right)$ is the velocity of the fluid at the position of the polymer blob, $\mathrm{d} t$ is the time-step used in the simulation, $\mathbf{F}_{a}$ is the body force on the polymer blob $a$ due to interaction with other blobs, $k_{B}$ is the Boltzmann constant, $T$ is the temperature, $\xi_{a}$ is the friction coefficient at the position of polymer blob $a$ and $\mathrm{dW}_{a}^{R}$ is a random fluctuation typical of a Brownian dynamics algorithm calculated in such a way that the fluctuation dissipation theorem is satisfied. The first term on the right hand side of the above equation essentially links the motion of the polymers to the flow of the background fluid. The position update for a fluid $i$ is given by $\mathrm{d} \mathbf{r}_{i}=\mathbf{v}_{i} \mathrm{~d} t$, where the update of velocity $\mathbf{v}_{i}$ is given by eq.(1.4).

$$
\begin{aligned}
\mathrm{d} \mathbf{v}_{i}= & -\frac{\mathrm{d} t}{m}\left[\sum_{j=1}^{N_{f}}\left(\frac{P_{i}}{\left(n_{i}^{f}\right)^{2}}+\frac{P_{j}}{\left(n_{j}^{f}\right)^{2}}\right) \frac{\mathrm{d} w^{f}}{\mathrm{~d} r}\left(r_{i j}\right) \frac{\mathbf{r}_{i j}}{r_{i j}}+\sum_{j=1}^{N_{f}} f_{i j} \mathbf{v}_{i j}\right]+\mathbf{g}_{i} \mathrm{~d} t \\
& +\frac{\mathrm{d} t}{m}\left[\sum_{a=1}^{N_{p}} \frac{w^{f}\left(r_{a i}\right)}{n_{i}^{f}} \mathbf{F}_{a}\right]+\sum_{j=1}^{N_{f}} \mathrm{~d} \mathbf{W}_{i j}^{v},
\end{aligned}
$$

where $m$ is the mass of the fluid blob $i, P_{i}$ is the pressure at the position of the fluid blob $i$ based on an equation of state that relates it to the local number density $n_{i}^{f}$ of the fluid blobs, $w^{f}$ is a normalized weight function, $\mathbf{r}_{i j}$ is the position of blob $i$ with respect to blob $j$, i.e. $\mathbf{r}_{i}-\mathbf{r}_{j}$ and similarly $\mathbf{v}_{i j}$ is the velocity of blob $i$ with respect to blob $j$, i.e. $\mathbf{v}_{i}-\mathbf{v}_{j} . f_{i j}$ is a symmetric function given in eq.(1.5).

$$
f\left(r_{i j}\right)= \begin{cases}-\left(\frac{2 \eta}{n_{i}^{f} n_{j}^{f}}\right) \frac{1}{r_{i j}} \frac{\mathrm{d} w^{f}}{\mathrm{~d} r}\left(r_{i j}\right) & \text { for } r_{i j} \leq R_{c} \\ 0 & \text { for } r_{i j} \geq R_{c}\end{cases}
$$


where $R_{c}$ is the cut-off, identical to the cut-off of the weight function $w^{f}(r)$ and $\eta$ is the solvent viscosity. $\mathbf{g}_{i}$ in eq.(1.4) is the acceleration due to body forces such as gravity etc. $\mathbf{F}_{a}$ is the polymer force that is through eq.(1.4) transmitted to the velocity of the fluid blobs, thereby providing the non-Newtonian contribution from the polymers to the fluid flow field. Finally, $\mathbf{d} \mathbf{W}_{i j}^{v}$ is a pairwise velocity fluctuation like in a Dissipative Particle Dynamics (DPD) simulation.[12, 13, 14] It is, in fact, identical to the velocity fluctuation calculated as per Smoothed Dissipative Particle Dynamics (SDPD), $[15,16]$ which is a modified version of DPD and SPH.

One of the advantages of using this model over the one described in Chapter 2 is that the resolution of the fluid can be chosen independently from the polymer concentration. This makes the method very efficient for higher polymer concentrations as the fluid resolution need only be good enough to accurately calculate the second order derivatives of the velocity needed for the solution of the Navier-Stokes equation using SPH. Furthermore, one is free to choose an equation of state for the fluid, which in our case we have chosen to be a pseudo-incompressible equation of state with a very high dependence of the pressure on the local density. This leads to the fluid being uniformly present throughout the system, whereas the polymer may exhibit cross-flow migration for instance in case of varying-shear rate environment. We observed this phenomenon with this model in addition to the characteristic flattening of the profile in plane Poiseuille flow. Observing the cross-flow migration effect would not have been possible with the previous model as the fluid is only present at the positions of the polymer blobs by default. Thus, this HCBD technique is a novel computational technique which couples the polymers exhibiting Brownian Dynamics with the fluid blobs moving as per Smoothed Particle Hydrodynamics.

In Chapter 4, we upgraded our polymer model to RaPiD with Finitely Extensible Nonlinear Elastic (FENE) dumbells, where the polymers are represented by two particles with a non-linear elastic spring between them, thereby adding internal elasticity to the model. We combined it with SPH using our HCBD technique that we described in Chapter 3. We observed shear banding for a certain set of parameters and we studied its time evolution in detail. In Chapter 5, we studied the flow of this 
model polymer solution through periodic arrays of cylindrical and cuboidal structures in two extreme flow directions. These geometries were chosen so as to mimic the different configurations that the fluid might encounter in any given porous medium such as an oil reservoir.

Thus, what we present in this thesis is the development of a new particle based coarse-grain simulation technique - Hydrodynamically Coupled Brownian Dynamics (HCBD). We also demonstrate its application for studying the flow of non-Newtonian fluids such as polymer solutions through porous media. In the future, this method can be extended to study the full multiphase flow of a non-Newtonian polymer solution driving the Newtonian oil trapped in the porous medium. This can potentially help further the understanding of polymer flow through a reservoir in the so-called polymer flooding application in Enhanced Oil Recovery. 


\section{Bibliography}

[1] R. B. Bird, W. E. Stewart, and E. N. Lightfoot, Transport Phenomena (Wiley, 2007).

[2] R. B. Bird, R. C. Armstrong, O. Hassager, and C. F. Curtiss, Dynamics of polymeric liquids, 1 (Wiley New York, 1977).

[3] S. Thomas. Oil \& Gas Science and Technology-Revue de l'IFP, 63, 9 (2008).

[4] A. Thomas, N. Gaillard, and C. Favero, Oil \& Gas Science and TechnologyRevue dIFP Energies nouvelles, 67, 887 (2012).

[5] J. T. Padding and W. J. Briels, The Journal of Chemical Physics, 141, 244108 (2014).

[6] V. R. Ahuja, J. van der Gucht and W. J. Briels, The Journal of Chemical Physics, 145, 194903 (2016).

[7] V. R. Ahuja, J. van der Gucht, and W. J. Briels, The Journal of Chemical Physics, 148, 034902 (2018).

[8] A. van den Noort, W. K. den Otter, and W. J. Briels, Europhysics Letters, 80, 28003 (2007).

[9] W. J. Briels. Soft Matter, 5, 4401 (2009).

[10] J. J. Monaghan, Annual Review of Astronomy and Astrophysics, 30, 543 (1992).

[11] J. J. Monaghan, Reports on Progress in Physics, 68, 1703 (2005).

[12] P. J. Hoogerbrugge and J. M. V. A. Koelman, Europhysics Letters, 19, 155 (1992).

[13] J. M. V. A. Koelman and P. J. Hoogerbrugge, Europhysics Letters, 21, 363, (1993). 
[14] P. Español and P. Warren, Europhysics Letters, 30, 191 (1995).

[15] P. Español and M. Revenga, Physical Review E, 67, 026705 (2003).

[16] A, Vázquez-Quesada, M. Ellero, and P. Español, The Journal of Chemical Physics, 130, 034901 (2009). 


\section{Two-way coupling of polymers with implicit solvent}

We present a coarse-grained particle-based simulation technique for modeling flow of complex soft matter fluids such as polymer solutions in the presence of solid interfaces. In our coarse-grained description of the system, we track the motion of polymer molecules using their centers-of-mass as our coarse-grain co-ordinates and also keep track of another set of variables that describe the background flow field. The coarse-grain motion is thus influenced not only by the interactions based on appropriate potentials used to model the particular polymer system of interest and the random kicks associated with thermal fluctuations, but also by the motion of the background fluid. In order to couple the motion of the coarse-grain co-ordinates with the background fluid motion, we use a Galilean invariant, first order Brownian dynamics algorithm developed by Padding and Briels [J. Chem. Phys. 141, 244108 (2014)], which on the one hand draws inspiration from Smoothed Particle Hydrodynamics in a way that the motion of the background fluid is efficiently calculated based on a discretization of the Navier-Stokes equation at the positions of the coarse-grain coordinates where it is actually needed, but also differs from it because of the inclusion of thermal fluctuations by having momentum-conserving pairwise stochastic updates. In this chapter, we make a few modifications to this algorithm and introduce a new parameter viz. a friction coefficient associated with the background fluid and analyze the relationship of the model parameters with the dynamic properties of the system. We also test this algorithm for flow in the presence of solid interfaces to show that appropriate boundary conditions can be imposed at solid-fluid interfaces by using artificial particles embedded in the solid walls which offer friction to the real fluid particles in the vicinity of the wall. We have tested our method using a model system of a star polymer solution at the overlap concentration. ${ }^{1}$

\footnotetext{
${ }^{1}$ This chapter has been published as V. R. Ahuja, J. van der Gucht, and W. J. Briels, J. Chem. Phys, 145(19), 194903 (2016).
} 


\subsection{Introduction}

The flow of complex soft matter involving mesoscopic particles can be described, in principle, by simultaneously solving the coupled deterministic equations of motion of all the atoms present in the system.[1,2] However, when dealing with processes occurring in soft matter systems over substantially longer length and time scales, typically several orders of magnitude higher than those occurring at the atomic level, this becomes computationally very expensive, rendering it practically impossible to solve the problem in this manner in a reasonable time-frame even with the state-of-the-art computers available today. Therefore, a computationally efficient solution is to simplify the description by lumping groups of atoms into coarse-grain sites which then interact with each other through effective interactions, thereby integrating out several internal degrees of freedom and making the problem tractable. [3, 4] Although it might seem too simplistic, it is interesting to notice that several phenomena in complex soft matter systems such as polymer melts, polymer solutions, worm-like-micellar solutions etc. have actually been studied by restricting the focus just to the motion of the centers-of-mass of the mesoscopic particles therein.[5, 6, 7, 8, 9, 10] It is needless to say that one has to be conscious of the fact that when modeling a system with coarsegrain simulations using the positions of the centers-of-mass, several internal degrees of freedom have been eliminated, which need to be accounted for. For instance, the same set of positions of these centers-of-mass could have a different time evolution because of a different configuration of the eliminated degrees of freedom. Hence, the coarse-grain system can no longer be treated as deterministic. This can be achieved by updating the positions of the centers-of-mass of the mesoscopic particles through a stochastic differential equation such as Langevin or Brownian dynamics.

At the outset, we would like to clarify what we refer to as Langevin and Brownian dynamics throughout this chapter. According to the terminology that we have employed, the motion of a mesoscopic particle moving through a stationary background according to Langevin dynamics is given by a second-order stochastic differential equation where the total force acting on the mesoscopic particle is given by a sum 
of three forces viz. a driving force due to the interaction with other mesoscopic particles or an external force field, a frictional force proportional to the velocity of the particle but acting against it, and a random force, whose properties are calculated based on the fluctuation dissipation theorem, thereby serving as a thermostat. Dissipative Particle Dynamics (DPD),[11, 12, 13] for instance, uses a framework based on Langevin dynamics. However, for highly overdamped soft matter systems, where friction coefficients are large, the timescale over which the velocities get thermalized is much shorter than the timescale over which the positions of the particles change to any significant extent. In such cases, one can average over the velocities and use instead a first order stochastic differential equation to update the position of the particles, henceforth referred to as Brownian dynamics.[14, 15] Several phenomena in soft matter systems, ranging from viscoelasticity in polymer solutions, shear thinning behavior, alignment of colloids in viscoelastic fluids, dynamics of proteins etc have been studied using a framework based on Brownian dynamics or its variations.[8, 9, 10, 16, 17, 18, 19, 20]

In order to study flow behavior of soft matter systems, the standard Brownian dynamics equations must be modified by the addition of a term that accounts for the velocity of the background material so that the friction is applied to the motion of the particles relative to the background velocity field. It is straightforward to do this if the background flow field is known a priori. However, for flows of soft matter systems through complex geometries involving solid interfaces, the flow field can be very complex and is not known a priori. The velocity of the background material at the position of the particle may be computed by averaging the drift velocity of the other particles in the vicinity of the particle itself. This has been done previously for the special case of shear flow of soft matter systems, where the velocity of the background material was calculated by spatio-temporally averaging the velocity of the particles in flow-oriented layers to study shear banding.[21, 22, 23, 24, 25]

A more general momentum-conserving algorithm for modeling self-developing flows of complex fluids in any flow situation has been developed recently.[26] Using this algorithm, friction is applied to the relative motion of the coarse-grained particles 
with respect to the background fluid. A separate set of variables is used for describing the background fluid velocities, which are updated based on a discretization of the Navier-Stokes equation at the positions of the coarse-grain coordinates, in a way similar to Smoothed Particle Hydrodynamics (SPH).[27, 28, 29, 30, 31] This obviates the need to calculate the entire background flow field and instead calculates the flow field only at the positions of the coarse-grain coordinates, where it is actually needed. The background velocity at the position of a coarse-grained particle is also influenced by the forces acting on the coarse-grained particle due to its interaction with other coarse-grained particles in its vicinity. These forces are immediately transmitted to the background flow field using a friction coefficient associated with the background fluid, which is an additional parameter we have introduced in the algorithm; however, the background material retains memory of this force, which gradually fades away with a characteristic time constant. The characteristic time constant and the friction coefficients are input parameters for our simulations which can, in principle, be calculated from the physical properties of the system measured experimentally or obtained from independent simulations. The friction coefficient associated with the coarse-grain motion may be calculated from the diffusion coefficient of the particles in the system. In this chapter, however, since the focus is model development, we have not used real values obtained from experimental data of physical properties but rather non-dimensionalized our results with respect to a basis set.

Since the motion of the coarse-grain particles influences the background flow field by transmitting the inter-particle force to it and the background flow field in turn influences the frictional force offered to the coarse-grain particles, this is a two-way coupling algorithm. In other words, the information about the background flow field is obtained effectively by spatio-temporally averaging the velocities in the vicinity of the coarse-grain coordinates, which is then used to calculate the friction for these coarse-grained particles relative to this background flow field. Furthermore, the background velocity is updated in a manner that conserves momentum pairwise. In this chapter we analyze this model, furthering the understanding of the relationship of the model parameters with the dynamic properties of the system such as the mean 
squared displacement and the shear relaxation modulus. We discuss additional insights that we have developed, particularly related to the friction coefficient associated with the background fluid. We have also derived an approximate expression for the mean squared displacement of the coarse-grained particles for a simple case of non-interacting particles.

Although initially developed independently by Padding and Briels,[26] the momentum conserving algorithm bears similarity to an existing modeling technique called Smoothed Dissipative Particle Dynamics (SDPD),[32] which also incorporates thermal fluctuations in the standard SPH approach. SDPD has been shown to obey proper scaling with varying resolution for Brownian motion of a colloidal particle as well as a polymer molecule in suspension.[33] The method has recently been used to simulate polymeric liquids, $[34,35]$ where polymer molecules are represented as linear chains of beads connected to each other with springs in the presence of other fluid elements. The main difference between their approach and ours is that we discretize the Navier-Stokes equation for computing the velocity of the background fluid on the same nodes which also represent the centers-of-mass of the coarse-grained polymer molecules, thereby making our approach computationally more efficient. It is also worth mentioning that several improvements that have been suggested to SDPD, like incorporating angular momentum conservation, [36, 37] can also be easily incorporated in our technique if it is employed for applications where this might be important.[38]

The discussion so far has been restricted to bulk flow i.e. flow in the absence of interfaces or in other words, flow occurring in the bulk far away from any interfaces. If the algorithm is to be applied to study the flow of soft matter systems through confined spaces involving solid interfaces, then it is also necessary to impose appropriate boundary conditions at the solid-fluid interfaces which is an important objective of the work that we present here. There is significant ongoing work in this direction. Several techniques for implementing boundary conditions for coarse-grain techniques such as Smoothed Particle Hydrodynamics and Dissipative Particle Dynamics have been developed.[39, 40, 41, 42] We have used the technique of incorporating ran- 
domly placed 'artificial particles' in the wall and implementing boundary conditions used by Morris et al.[39] for modeling low Reynolds number incompressible flows using Smoothed Particle Hydrodynamics. The choice of the term 'artificial particles' here is simply motivated by the fact that these particles do not obey any real dynamics. Rather, the sole function of these artificial particles is to offer friction to the real fluid particles in the vicinity of the wall by acting like 'frozen particles'. However the position of these artificial particles is not fixed in the wall. In order that the real particles feel a uniform wall i.e. to ensure that the random positions of these artificial particles in the wall do not bias the flow field in any way, the position of these artificial particles is constantly randomized within the wall. This obviates the need to use a high density of these particles in order to maintain the uniformity of the wall. Thus, we present a technique for modeling flow of complex soft matter fluids using a Galilean invariant algorithm in the presence of solid interfaces.

\subsection{Model development}

\subsubsection{Update of positions}

The equation of motion for the mesoscopic particle $i$ in a moving background using Brownian dynamics is given by:

$$
\mathrm{d} \mathbf{R}_{i}(t)=\mathbf{v}_{i}(t) \mathrm{d} t+\frac{\mathbf{F}_{i}(t)}{\xi_{i}} \mathrm{~d} t+k_{B} T \frac{\partial}{\partial \mathbf{R}_{i}}\left(\frac{1}{\xi_{i}}\right) \mathrm{d} t+\mathrm{d} \mathbf{W}_{i}^{R}(t),
$$

where we must clarify that the first term on the right hand side of the above equation, i.e. $\mathbf{v}_{i}(t)$, is not the velocity of the mesoscopic particle $i$ itself; rather it is the velocity of the background material at the position of the mesoscopic particle, relative to which the particle experiences friction. The second term on the right hand side denotes the contribution from the overall force $\mathbf{F}_{i}$ experienced by particle $i$ including the external force field as well as the interaction with other mesoscopic particles. It must be noted that the friction coefficient $\xi_{i}$ has been assumed to be a constant in this chapter for the sake of simplicity, thereby obviating the need to account for its positional variation shown in the third term on the right hand side. The fourth term on the right hand side 
i.e. $\mathrm{d}_{\mathbf{i}}^{R}(t)$ is a random displacement typical of Brownian dynamics simulations. The properties of the random fluctuations in the position updates are calculated based on the fluctuation dissipation theorem and are thus defined as follows:

$$
\left\langle\mathrm{d} \mathbf{W}_{i}^{R} \mathrm{~d} \mathbf{W}_{j}^{R}\right\rangle=\frac{2 k_{B} T}{\xi_{i}} \delta_{i j} \mathrm{~d} t \mathbf{I} .
$$

\subsubsection{Update of velocities}

If the background flow field is known a priori, then it can be plugged into Eq.(2.1) and the problem can be solved quite easily. However, the flow field for soft matter fluids flowing through complex geometries is typically quite complex and is not known $a$ priori. To calculate the background flow field based on the motion of the coarse grain coordinates, we use the momentum conserving Galilean invariant two-way coupling scheme proposed by Padding and Briels[26] with our own modifications. This algorithm couples the motion of the coarse-grain coordinates and the background flow field with each other. In this section, we briefly describe this algorithm and its development. The scheme for updating velocities in this algorithm is not based on a microscopic description but rather a phenomenological description. Consider the Navier-Stokes equation for the velocity field $\mathbf{v}(\mathbf{r})$ of a Newtonian liquid having kinematic viscosity $v$ :

$$
\frac{\mathrm{D} \mathbf{v}}{\mathrm{D} t}(\mathbf{r})=v \nabla^{2} \mathbf{v}(\mathbf{r})+\mathbf{g}(\mathbf{r}),
$$

where $\mathrm{D} / \mathrm{D} t$ is the material derivative and $\mathbf{g}(\mathbf{r})$ is the acceleration due to body forces. Now, instead of solving the above equation on an Eularian grid, inspired by Smoothed Particle Hydrodynamics (SPH),[27, 28, 29, 30, 31] we calculate the velocity of the background material only at the positions of the particles, which is where it is actually needed as can be seen from Eq.(2.1). Thus, we have:

$$
\frac{\mathrm{D} \mathbf{v}}{\mathrm{D} t}\left(\mathbf{R}_{i}\right)=\frac{\mathrm{d} \mathbf{v}_{i}}{\mathrm{~d} t}
$$

The acceleration due to body forces for the background fluid is due to the force $\mathbf{F}_{i}$ acting on the coarse-grained particles, which is immediately transmitted to the back- 
ground fluid using an effective mass $m_{i}$ as follows:

$$
\mathbf{g}\left(\mathbf{R}_{i}\right)=\frac{\mathbf{F}_{i}}{m_{i}}
$$

For calculating the Laplacian of the velocity field i.e. $\nabla^{2} \mathbf{v}(\mathbf{r})$, a finite-difference like form has been employed, which is a symmetrized version of the form originally proposed by Brookshaw.[43] Thus, we have:

$$
v \nabla^{2} \mathbf{v}\left(\mathbf{R}_{i}\right)=v \sum_{j=1}^{N} m_{j}\left(\frac{1}{\rho_{i}}+\frac{1}{\rho_{j}}\right)\left(\mathbf{v}_{i}-\mathbf{v}_{j}\right) \frac{1}{R_{i j}} \frac{\mathrm{d} w}{\mathrm{~d} r}\left(R_{i j}\right),
$$

where $R_{i j}$ is the distance between particles $i$ and $j$, the function $w(r)$ is a normalized dimensionless weight function defined later and $\rho_{i}$ is the effective mass density for the background fluid in the vicinity of particle $i$ defined as follows:

$$
\rho_{i}=\frac{\sum_{j=1}^{N} m_{j} w\left(R_{i j}\right)}{1+w(0) / \rho^{\#}},
$$

where the sum in the numerator includes the self term $j=i$. We must point out that we have changed the definition of $\rho_{i}$ from the original definition proposed in the algorithm by Padding and Briels [26] in order to ensure proper normalization ${ }^{2}$ so that for a homogeneous solution, we have $\rho=m \rho^{\#}$, where $\rho^{\#}$ is the number density of the particles.

Putting together equations (2.3),(2.4), (2.5) and (2.6), and including a random term as per the fluctuation dissipation theorem, we arrive at the following equation that we have used in our simulations for the update of the velocities:

$$
\mathrm{d} \mathbf{v}_{i}(t)=\frac{\mathbf{F}_{i}(t)}{m_{i}} \mathrm{~d} t+\sum_{j=1}^{N} \frac{f_{i j}}{\tau}\left(\mathbf{v}_{j}(t)-\mathbf{v}_{i}(t)\right) \mathrm{d} t+\sum_{j=1}^{N} \mathrm{~d} \mathbf{W}_{i j}^{v},
$$

where $f_{i j}$, which is shorthand for $f\left(R_{i j}\right)$, is a normalized dimensionless weight function and $\tau$ is a characteristic time constant associated with the background fluid, both of which will be defined later, such that their ratio is given by:

$$
\begin{aligned}
& \frac{f_{i j}}{\tau}=-v m_{j}\left(\frac{1}{\rho_{i}}+\frac{1}{\rho_{j}}\right) \frac{1}{R_{i j}} \frac{\mathrm{d} w}{\mathrm{~d} r}\left(R_{i j}\right) \quad\left(r \leq R_{c}\right) . \\
& { }^{2} \text { For a homogeneous solution, } \sum_{j=1, j \neq i}^{N} w\left(R_{i j}\right)=\int d^{3} r \rho^{\#} w(r)=\rho^{\#} \text {, and hence, } \sum_{j=1}^{N} m w\left(R_{i j}\right)=m\left(w(0)+\sum_{j=1, j \neq i}^{N} w\left(R_{i j}\right)\right)= \\
& m \rho^{\#}\left(1+w(0) / \rho^{\#}\right), \text { from which the result follows }
\end{aligned}
$$


The last term on the right hand side of Eq.(2.8) is a random contribution to the velocity update, defined pairwise in an antisymmetric manner such that $m_{i} \mathrm{~d} \mathbf{W}_{i j}^{v}=-m_{j} \mathrm{~d} \mathbf{W}_{j i}^{v}$ so that the velocity updates are momentum conserving. The properties of these velocity fluctuations $\mathrm{d} \mathbf{W}_{i j}^{v}$ have been calculated in a way that the probability distribution of the coarse-grain coordinates and velocities at steady state matches with the expected equilibrium distribution. For a detailed derivation starting with the Chapman-Kolmogorov equations leading to a Fokker-Planck equation for the evolution of the probability distribution, we refer to the appendix of the paper by Padding and Briels.[26] Accordingly, we have:

$$
\begin{aligned}
\left\langle\mathrm{d} \mathbf{W}_{i j}^{v} \mathrm{~d} \mathbf{W}_{i j}^{v}\right\rangle & =\frac{2 k_{B} T}{m_{i}} \frac{f_{i j}}{\tau} \mathrm{d} t \mathbf{I}, \\
\left\langle\mathrm{d} \mathbf{W}_{i k}^{v} \mathrm{~d} \mathbf{W}_{j l}^{v}\right\rangle & =\mathbf{0} \\
\left\langle\mathrm{d} \mathbf{W}_{i}^{R} \mathrm{~d} \mathbf{W}_{j k}^{v}\right\rangle & =\mathbf{0} .
\end{aligned} \quad(i k \neq j l \wedge i k \neq l j),
$$

If we multiply Eq.(2.8) throughout by the effective mass $m_{i}$, we obtain:

$$
m_{i} \mathrm{~d} \mathbf{v}_{i}(t)=\mathbf{F}_{i}(t) \mathrm{d} t+\sum_{j=1}^{N} f_{i j} \frac{m_{i}}{\tau}\left(\mathbf{v}_{j}(t)-\mathbf{v}_{i}(t)\right) \mathrm{d} t+m_{i} \sum_{j=1}^{N} \mathrm{~d} \mathbf{W}_{i j}^{v}
$$

Owing to the resemblance of the above rearranged equation with the Langevin equation, we naturally define the friction coefficient associated with the background fluid in an analogous manner as follows:

$$
\xi_{i}^{\prime}=\frac{m_{i}}{\tau}
$$

It must be noted that in our simulations, all the particles have an identical mass and hence the friction coefficient $\xi_{i}^{\prime}$ has been assumed to be a constant in this chapter. However, contrary to the paper by Padding and Briels,[26] we do not set $\xi^{\prime}$ identically equal to $\xi$. Rather, in this chapter, we have investigated the effect of this friction coefficient $\xi^{\prime}$ on the dynamics of the system, which is actually very significant. The numerical value for $\xi^{\prime}$ can be indirectly calculated based on the value of the mass, which can be calculated as the ratio of the mass density of the system to the number density of the particles and the value of the characteristic time constant, which can 
be calculated based on the kinematic viscosity of the system, as will be clear later. In this chapter, however, since our focus is model development, we have not used real values obtained from experimental data but rather non-dimensionalized our results with respect to a basis set, which will be defined later.

\subsubsection{Interaction with solid walls}

For studying confined flows i.e. flows in the presence of solid interfaces, it is important to take into account the interaction of the fluid with the solid walls establishing appropriate boundary conditions. We have tested our model for applying the no-slip boundary conditions with a test case where we have a star polymer solution flowing between two infinite, parallel solid walls. Our model for the solid-fluid interactions consists of incorporating 'artificial' particles in the wall and implementing Morris boundary conditions [39], which provide the necessary friction to the real particles in order to obtain no-slip boundary conditions at the solid-fluid interface. The Morris boundary conditions involve assigning an artificial velocity to these artificial particles for every pairwise interaction with a real particle, such that the interpolated velocity at the solid interface is zero. This is achieved by setting the artificial velocity of an artificial particle $B$ at a perpendicular distance $d_{B}$ from the solid interface for its interaction with real particle $A$ at a perpendicular distance $d_{A}$ from the solid interface as follows:

$$
\mathbf{v}_{B}=-\left(d_{B} / d_{A}\right) \mathbf{v}_{A}
$$

It must be emphasized this is an artificial velocity in the sense that this velocity is not used to evolve the position of the artificial particle. Rather, it is used for calculating the difference in the velocity of the particles needed for the velocity update of the real particle, as shown below:

$$
\mathbf{v}_{A}-\mathbf{v}_{B}=\beta \mathbf{v}_{A},
$$

where the value of $\beta$ is restricted with an upper bound for practical considerations for the eventuality that the particle $A$ may approach very close to the solid-fluid boundary, 
as shown below:

$$
\beta=\min \left(\beta_{\text {max }}, 1+\frac{d_{B}}{d_{A}}\right),
$$

where $\beta_{\max }$ is chosen to be 1.5 in our simulations as suggested by Morris et al.[39] Furthermore, the velocity fluctuation term for the interaction between real and artificial particles is also augmented with a factor of $\sqrt{\beta}$, which prevents cooling of the fluid near the wall. The positions of the artificial particles within the wall are selected randomly at every time-step in order to maintain the uniformity of the wall. This has proven to be very effective, thus obviating the need for using a higher density of these artificial particles inside the wall which would have been computationally more intensive. So in our simulations, we have chosen the density of artificial particles embedded in the wall to be the same as the density of the real particles. Besides, we also use a repulsive potential which keeps the real particles from penetrating into the wall, which is the region in which the artificial particles are distributed. For the repulsive potential, we have chosen a Gaussian function with two parameters as shown below:

$$
\phi^{r e p}\left(r_{i}\right)=a \exp \left(-b r_{i}^{2}\right)
$$

where $r_{i}$ is the perpendicular distance of particle $i$ from a plane situated within the wall at a distance of $1.2 \sigma$ from the interface, $a$ is a parameter chosen to be $300 k_{B} T$ and $b$ is a parameter chosen to be $2 \sigma^{-2}$. These parameters have been selected such that the real particles do not penetrate the $1.5 \sigma$ thick wall embedded with artificial particles on top and bottom of the channel of width $10 \sigma$ in which the real particles are present.

\subsection{Test System and Parameters}

\subsubsection{Test system}

We have tested our model with a star polymer solution at the overlap concentration. The behavior of star polymers can vary ranging from ultrasoft to nearly hard colloid like behavior as their interpenetrability depends on their functionality, which is 
defined as the number of linear chains (arms) bound to the central core of the star polymer. Owing to their wide-ranging interactions, they can be used to study a range of colloidal properties for different interactions.[44]. At an intermediate functionality, due to the radial variation of the monomer concentration,[45] each star polymer can be treated as soft repulsive sphere with a small central core and a corona of grafted chains around it. We use a potential $V_{s s}$ for the interaction between the particles in our simulation, which has been used for modeling star polymers in the past and has also been verified by scattering experiments.[7] It is defined as follows:

$$
\begin{array}{rlrl}
\frac{V_{s s}(r)}{k_{B} T}=\frac{5}{18} f^{3 / 2}\left[-\ln \left(\frac{r}{\sigma}\right)+\frac{1}{1+\sqrt{f} / 2}\right] & & \text { for } r \leq \sigma, \\
& =\frac{5}{18} f^{3 / 2} \frac{1}{1+\sqrt{f} / 2} \frac{\sigma}{r} \exp \left[-\frac{\sqrt{f}}{2 \sigma}(r-\sigma)\right] & & \text { for } \sigma<r \leq r_{c},
\end{array}
$$

where $f$ is the functionality, $r$ is the distance between the particles, $\sigma$ is the effective corona diameter and $r_{c}$ is the cut-off radius at which the potential is truncated, which is chosen such that the forces are negligible beyond this distance.

\subsubsection{Definitions of weight functions and the characteristic time constant}

We have chosen a normalized weight function $w(r)$ from the SPH literature that smoothly decays to zero as $r$ approaches the cut-off radius $R_{c}$, given as follows:

$$
w(r)=\frac{21}{2 \pi R_{c}^{3}}\left(1-\frac{r}{R_{c}}\right)^{4}\left(4 \frac{r}{R_{c}}+1\right) \quad\left(r \leq R_{c}\right) .
$$

Thus, in accordance with Eq.(2.9) and Eq.(2.21), we define the normalized weight function $f_{i j}$ and the characteristic time constant $\tau$ as follows:

$$
\begin{aligned}
& f_{i j}=-\frac{R_{c}^{2}}{28} m_{j}\left(\frac{1}{\rho_{i}}+\frac{1}{\rho_{j}}\right) \frac{1}{R_{i j}} \frac{\mathrm{d} w}{\mathrm{~d} r}\left(R_{i j}\right) \quad\left(r \leq R_{c}\right), \\
& \tau=\frac{R_{c}^{2}}{28 v},
\end{aligned}
$$

where the coefficient $R_{c}^{2} / 28$ appears due to normalization of $f_{i j}$. Simplifying further, we obtain the following expression for $f_{i j}$ :

$$
f_{i j}=\frac{15}{2 \pi R_{c}^{3}} m_{j}\left(\frac{1}{\rho_{i}}+\frac{1}{\rho_{j}}\right)\left(1-\frac{R_{i j}}{R_{c}}\right)^{3} \quad\left(r \leq R_{c}\right) .
$$




\subsubsection{Parameter Values}

We have expressed all the variables in terms of a basis set consisting of the following three quantities : (1) Thermal energy $k_{B} T$ (2) Diffusion coefficient $D_{o}$ and (3) Effective corona diameter $\sigma$. The friction coefficient $\xi$ is assumed to be constant for the sake of simplicity and is given by $\xi=k_{B} T / D_{0}$. Furthermore, we have also assumed a uniform mass which implies a uniform $\xi^{\prime}$ for all the particles in any given simulation. However we have performed simulations with different values of $\xi^{\prime}$ by systematically varying the ratio $\xi^{\prime} / \xi$ ranging from 1 to 100 . We have used a timestep $\mathrm{d} t=10^{-4} \sigma^{2} / D_{0}$ for all our simulations. We have performed several simulations with different values of $\tau$ ranging form $1.0 \times 10^{-3} \sigma^{2} / D_{0}$ to $1.0 \times 10^{1} \sigma^{2} / D_{0}$ ensuring that we always maintain $\tau>10 \mathrm{~d} t$ for stability of the algorithm. In our simulations, we have worked with star polymers having a functionality $f=128$. For operating at the overlap concentration $\left(c=c^{*}\right)$, the number density of the star polymers has been fixed to $0.24 \sigma^{-3}$.[46] For the confined flow simulations, the density of artificial particles inside the wall is the same as the density of the real particles. So in a cubical simulation box having a side of $13 \sigma$, we have 524 particles. We have used an effective cut-off radius $r_{c}=2.5 \sigma$ for the potential beyond which the forces are weak enough to be ignored. We have chosen the same cut-off distance $R_{c}$ for our weight function $w(r)$ as well, which leads to approximately 15 particles within a sphere of radius $R_{c}$, ensuring that we have an accurate enough estimate of the Laplacian of the velocity field.

\subsection{Results and Discussion}

\subsubsection{Bulk flow simulations in quiescent state}

In this section, we present the results for simulations of our test system i.e. a star polymer solution at overlap concentration performed in the absence of any solid interfaces to simulate flows far away from any walls, i.e. in the bulk, in a quiescent state. We have studied the effect of our model parameters on some static and dynamic 
properties of the system and compared the results with standard Brownian dynamics simulations in the absence of a background flow field (referred to as 'standard BD' in the graphs). All the static properties remain unaffected by the Galilean invariant algorithm.[26] As can be seen from Fig.2.1, we have verified that the radial distribution function $g(r)$ of the particles for several different values of the parameters of the algorithm is identical to the radial distribution function of the particles in the absence of a background flow field.

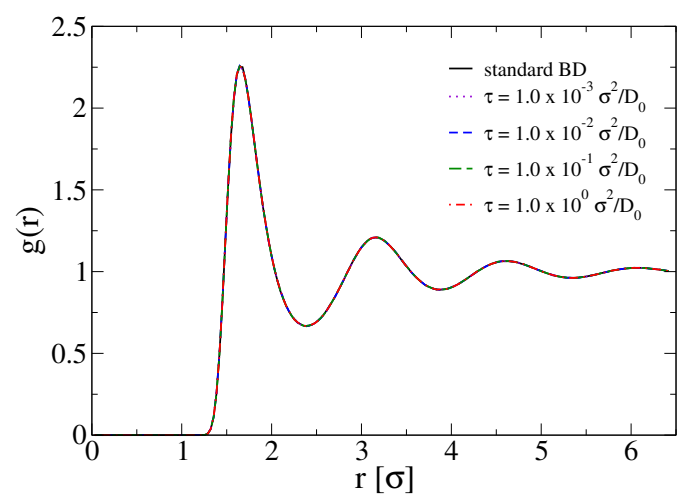

Figure 2.1: Radial distribution function of the particles obtained from standard Brownian dynamics simulation shown with black solid line vs. Brownian dynamics with the Galilean invariant algorithm for various values of the parameter $\tau$ (for any given value of $\xi^{\prime} / \xi$ ) shown with colored dotted lines.

It is interesting to note however that even though the static properties are unaffected by the Galilean invariant algorithm, we have found that the dynamic properties are affected by it. We have studied the effect on two dynamic properties viz. the shear relaxation modulus $G(t)$ and the mean squared displacement (MSD) of the particles by a systematic variation of the system parameters $\tau$ and $\xi^{\prime}$. We have found that the effect on the dynamic properties due to variation of $\tau$ is significant and directly depends on the value of $\xi^{\prime}$ or vice versa. Since we have implicitly chosen $\xi$ as a basic unit as it is directly linked to $D_{0}$, we will discuss this effect in terms of the ratio $\xi^{\prime} / \xi$. The shear relaxation modulus $G(t)$ has been computed as an autocorrelation of spontaneous shear stress fluctuations in an equilibrium simulation, as shown in the 
following equations:

$$
\begin{aligned}
G(t) & =\frac{V}{k_{B} T}\left\langle S_{x y}^{p p}(t) S_{x y}^{p p}(0)\right\rangle, \\
S_{x y}^{p p}(t) & =-\frac{1}{V} \sum_{i<j}\left(x_{i}-x_{j}\right) F_{y, i j},
\end{aligned}
$$

where $V$ denotes the volume of the box and $S_{x y}^{p p}$ is the $x y$-component of the microscopic particle stress tensor which is calculated from $F_{y, i j}$, the $y$-component of the force on particle $i$ due to its interaction with particle $j$. The effect of the model parameters on the shear relaxation modulus $G(t)$ of the particles is shown in Fig.2.2.

The mean squared displacement has been calculated as follows:

$$
\operatorname{MSD}(t)=\left\langle(\mathbf{r}(t)-\mathbf{r}(0))^{2}\right\rangle
$$

The effect of the model parameters on the mean squared displacement (MSD) of the particles is shown in Fig.2.3.

It can be observed from the graphs of the shear relaxation modulus $G(t)$ as shown in Fig.2.2 that the higher the value of $\tau$, the slower the stresses relax until eventually approaching the standard Brownian dynamics limit at very high values of $\tau$. Furthermore, the effect of variation of $\tau$ is more pronounced for smaller values of the ratio $\xi^{\prime} / \xi$ and it becomes almost insignificant at high values of the ratio $\xi^{\prime} / \xi$. A similar effect can be seen for the mean squared displacement of the particles.

As can be seen from Fig.2.3, the long term diffusion coefficient decreases with increasing value of $\tau$ until finally approaching the standard Brownian dynamics limit at very high values of $\tau$. 


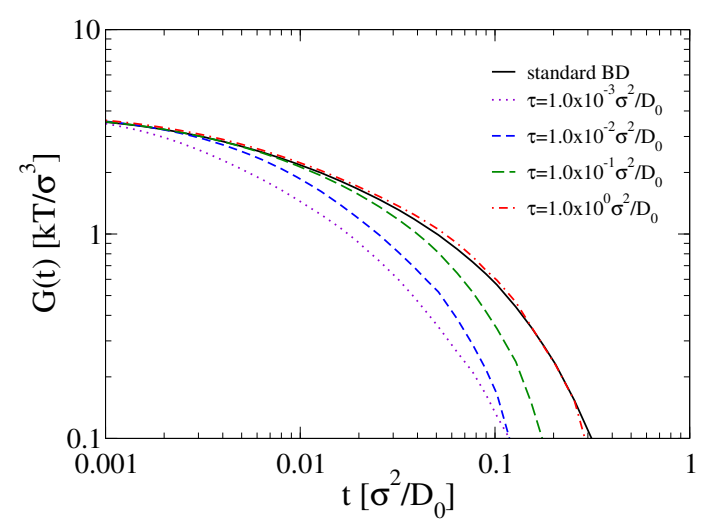

(a) $\xi^{\prime} / \xi=1$

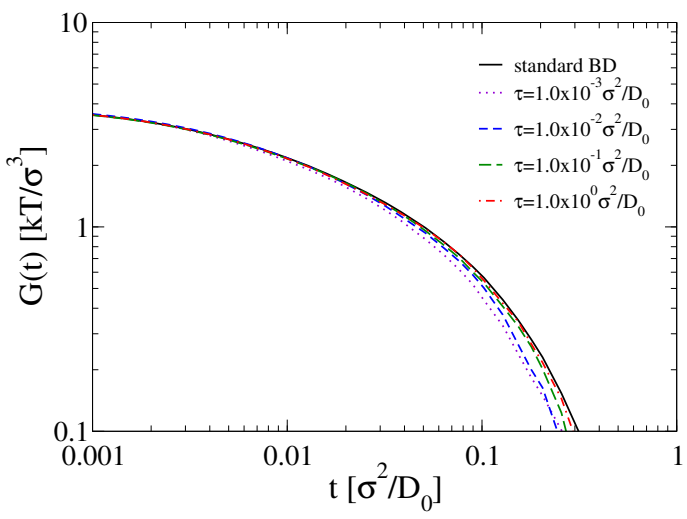

(b) $\xi^{\prime} / \xi=10$

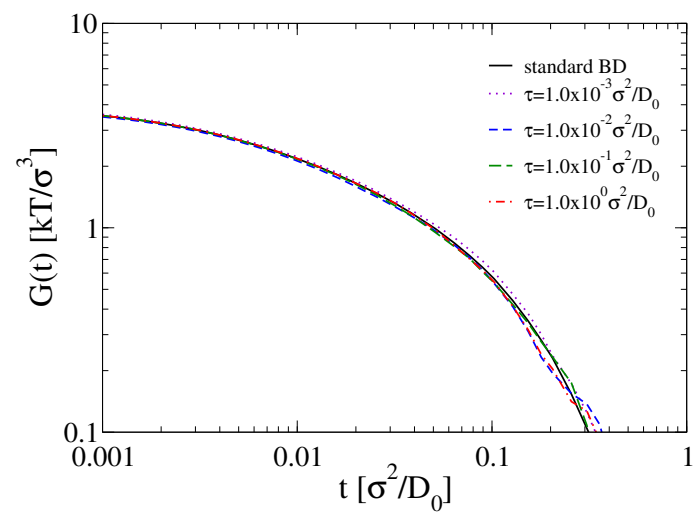

(c) $\xi^{\prime} / \xi=100$

Figure 2.2: Shear relaxation modulus of the particles using standard Brownian dynamics simulation shown with black solid line vs. using the Galilean invariant algorithm for various parameter values shown with colored dotted lines. 


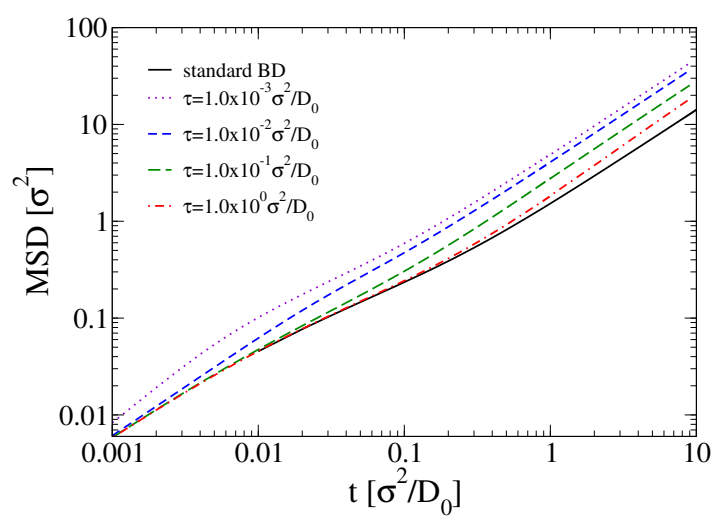

(a) $\xi^{\prime} / \xi=1$

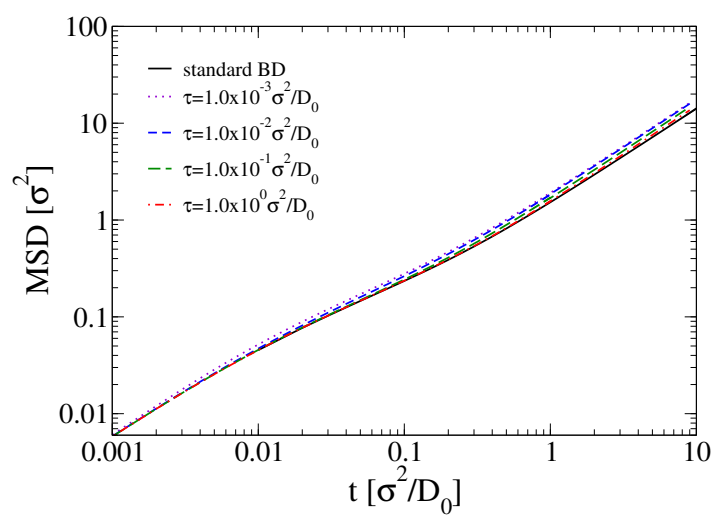

(b) $\xi^{\prime} / \xi=10$

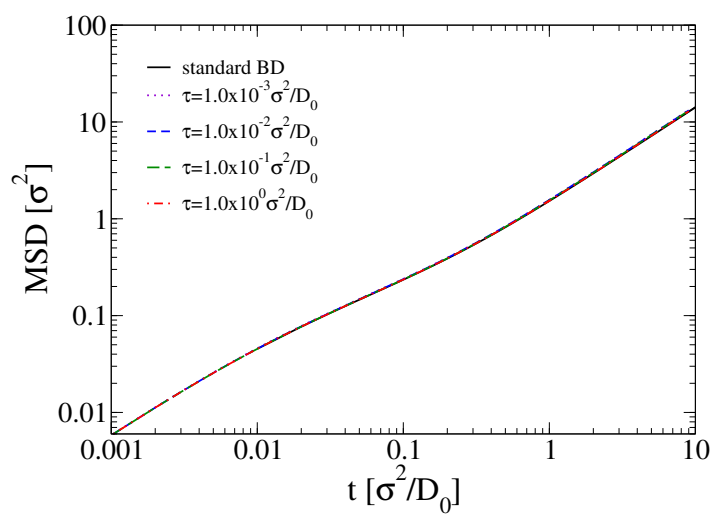

(c) $\xi^{\prime} / \xi=100$

Figure 2.3: Mean squared displacement of the particles using standard Brownian dynamics simulation shown with black solid line vs. using the Galilean invariant algorithm for various parameter values shown with colored dotted lines. 
Again, as in the case of the shear relaxation modulus, the effect is more pronounced at smaller values of the ratio $\xi^{\prime} / \xi$ and becomes almost insignificant at higher values of the ratio $\xi^{\prime} / \xi$. The effect of $\tau$ on the dynamic properties can be readily understood if we interpret $\tau$ as being indicative of the time over which the background velocity is averaged or in other words, the memory of the system. If we rewrite Eq.(2.8) using Eq.(2.14) and assume a homogeneous distribution of particles, we get:

$$
\mathbf{v}_{i}(t+\mathrm{d} t)=\left(1-\frac{\mathrm{d} t}{\tau}\right) \mathbf{v}_{i}(t)+\frac{\mathrm{d} t}{\tau}\left[\frac{\mathbf{F}_{i}(t)}{\xi}\left(\frac{\xi^{\prime}}{\xi}\right)^{-1}+\sum_{j=1}^{N} f_{i j} \mathbf{v}_{j}(t)\right]+\sum_{j=1}^{N} \mathrm{~d} \mathbf{W}_{i j}^{v}
$$

From this perspective, the background velocity in the vicinity of particle $i$ can be interpreted as being calculated based on spatio-temporal averaging.[26] Clearly, the characteristic time constant $\tau$ dictates over how many time-steps the system will average the background velocity or in other words what is the memory of the system. So if $\tau$ is very large, the background velocity gets averaged over such a long time that it becomes effectively negligible since we are dealing with the bulk in quiescent state (i.e. the velocities fluctuate about a long term average value of zero). The position updates then reduce to standard Brownian Dynamics.

Another interesting point here is that this effect of $\tau$ on the dynamic properties systematically diminishes as the value of the ratio $\xi^{\prime} / \xi$ is increased, until finally becoming almost insignificant at very large values of the ratio $\xi^{\prime} / \xi$. This is due to the fact that when $\xi^{\prime} \gg \xi$, the coupling between the coarse grain motion and the background flow field becomes insignificant which can be seen from Eq.(2.28) as the diminishing effect of the force transmitted to the background flow field when $\xi^{\prime} / \xi$ is very large. In other words, when the ratio $\xi^{\prime} / \xi$ is very large, the background flow field does not feel the interactions between the coarse-grain particles and vice versa, leading to the shear relaxation modulus and the mean squared displacement of the particles approaching the standard Brownian dynamics limit.

The model is thus very general and can be used for a diverse range of behaviors ranging from particles that are strongly affected by the fluctuations of the background to particles that are so weakly affected by the fluctuations of the background that they 
are almost oblivious of its existence. Since the diffusion coefficient is something that can be measured for a system experimentally, it could be used to calculate the parameters of the model. Therefore, we next derive an approximate expression for the mean squared displacement. It must be pointed out however that this derivation is approximate in the sense that it does not fully capture the effects due to all the model parameters, as it makes several assumptions.

Consider a simple case of non-interacting particles in the bulk in quiescent state i.e. a case where there are no forces on the particles except for the random kicks and velocity fluctuations. For a homogeneous solution where the friction coefficients of all particles are identical, the model dictates the following position and velocity updates respectively:

$$
\begin{aligned}
& \mathrm{d} \mathbf{R}_{i}(t)=\mathbf{v}_{i}(t) \mathrm{d} t+\mathrm{d} \mathbf{W}_{i}^{R}(t) \\
& \mathrm{d} \mathbf{v}_{i}(t)=\frac{1}{\tau} \sum_{j=1, j \neq i}^{N} f_{i j} \mathbf{v}_{j}(t) \mathrm{d} t-\frac{\mathbf{v}_{i}(t) \mathrm{d} t}{\tau} \sum_{j=1, j \neq i}^{N} f_{i j}+\sum_{j=1, j \neq i}^{N} \mathrm{~d} \mathbf{W}_{i j}^{v}(t) .
\end{aligned}
$$

For a homogeneous solution, $\sum_{j=1, j \neq i}^{N} f_{i j}$ averaged over a long term would be equal to unity for all particles. So as a simplifying assumption, we assume a constant average value of $\sum_{j=1, j \neq i}^{N} f_{i j}=1$, thereby also neglecting any correlations that it may have with the velocities. The above equation then can be alternatively written as an integral equation as follows:

$$
\mathbf{v}_{i}(t)=\mathbf{v}_{i}(0) e^{-t / \tau}+\int_{0}^{t} \mathrm{~d} t^{\prime} e^{-\left(t-t^{\prime}\right) / \tau}\left(\sum_{j=1, j \neq i}^{N} \frac{\mathrm{d} \mathbf{W}_{i j}^{v}\left(t^{\prime}\right)}{\mathrm{d} t}+\frac{1}{\tau} \sum_{j=1, j \neq i}^{N} f_{i j} \mathbf{v}_{j}\left(t^{\prime}\right)\right) .
$$

This equation may be solved in the usual way by iteration. Actually, since the second term in the integrand will be small, we expect that the zeroth order calculation by neglecting this term, will give a good result. For a detailed derivation, the reader may refer to the appendix. For the zeroth order calculation, we arrive at the following result:

$$
\left\langle\Delta \mathbf{R}_{i}(t)^{2}\right\rangle=\frac{6 k_{B} T}{\xi_{i}^{\prime}}\left[t-\tau\left(1-e^{-t / \tau}\right)\right]+\frac{6 k_{B} T}{\xi_{i}} t .
$$


After a sufficiently long time i.e. $t \gg \tau$, we get:

$$
\left\langle\Delta \mathbf{R}_{i}(t)^{2}\right\rangle=\frac{6 k_{B} T}{\xi_{i}^{\prime}} t+\frac{6 k_{B} T}{\xi_{i}} t .
$$

Thus, we can see from the above equation that the effect of the friction coefficient associated with the background on the dynamic properties of the system such as the mean squared displacement of the particles is significant and is in line with what we observed from the simulation results shown earlier. We can use Eq.(2.33) to set the value of the friction coefficient $\xi$ so as to obtain the correct diffusion coefficient of the particles in the system.

In order to compare our derivation results, we have performed simulations of particles with the potential $V_{s s}$ turned off so as to be as close to the assumptions made in the derivation as possible and the results are shown in Fig.2.4.

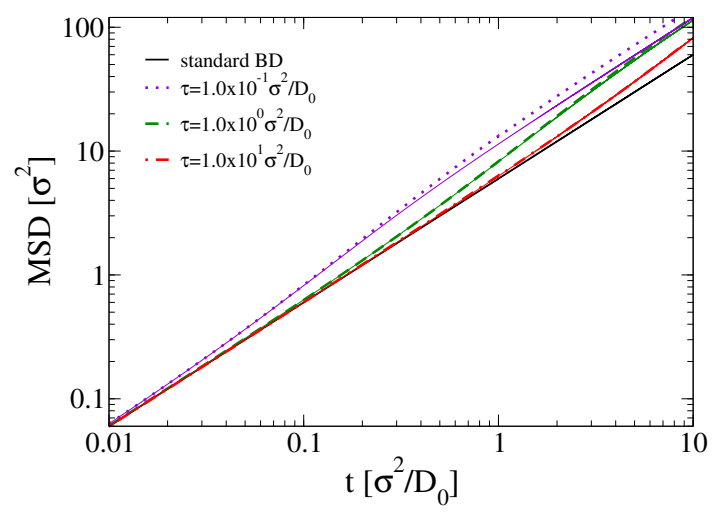

Figure 2.4: Comparison of the mean squared displacement of the non-interacting particles using the result from the derivation i.e. Eq.(2.32) with simulations for $\xi^{\prime} / \xi=1$. The colored dotted lines are simulation results, the colored solid lines are the analytical approximations from the derivation and the black solid line is for a standard Brownian dynamics simulation.

As we can see from Fig.2.4, the expression derived from theory satisfactorily predicts the mean squared displacement of the particles, particularly for large values of $\tau$. 


\subsubsection{Flow simulations with solid interfaces}

To test the model for flow in the presence of solid interfaces, we have performed simulations of the test system i.e. a star polymer solution flowing through a rectangular channel. The cubical simulation box with each side measuring $13 \sigma$, consists of a rectangular channel of width $10 \sigma$ that has been simulated by having two walls filled with artificial particles, each of thickness $1.5 \sigma$, placed within the box - one at the top and the other at the bottom. Periodic boundary conditions have been applied in all the directions including the vertical direction so that any particle approaching a wall effectively encounters a wall of $3 \sigma$, which is more than the cut-off radius $R_{c}$ of $2.5 \sigma$. This prevents any unrealistic interactions a particle near the bottom wall could have had with another particle near the top wall due to the periodic boundary conditions in the vertical direction. Alternatively, one can also have a wall of $2.5 \sigma$ thick on top and the bottom face without periodic boundary conditions in the vertical direction but this would involve using a greater number of artificial particles. In the simulations, flow has been induced in the positive $\mathrm{x}$-direction by using a gravity field $g$, which is essentially applied by applying a constant force $F_{x}$ to all the particles in the positive $\mathrm{x}$-direction. Now, this force may be applied to the particles and transmitted to the background fluid or alternatively the gravity field may just be applied to the background fluid through the force term in the update of the velocities without using it in the update of the positions. In either approach, similar velocity profiles are obtained. Here, we present the results for the case where the force $F_{x}$ is applied to the particles and transmitted to the background fluid.

In the simulation results that we have presented in this section, we have nondimensionalized the density, velocity and temperature profiles. The density profile has been non-dimensionalized using the bulk density $\rho^{\text {bulk}}$, which is essentially the ratio of the total mass of all particles to the total volume of the box. It is important to note that the density of the artificial particles within the channel is the same as the density of the real particles in the channel, which is equal to $\rho^{\text {bulk }}$. The velocity profile is calculated by dividing the box into 26 slabs and measuring the velocities 
of all the particles within each slab. We are mainly interested in the component of the velocity in the direction of the flow, which in our simulations was the positive $\mathrm{x}$-direction. So, for each slab, the distribution of $\mathrm{x}$-component of the velocities is calculated from a histogram, which is populated by the x-component of the velocities of all the particles in that slab. We run the simulation for $10^{7}$ time steps and use the last $9 \times 10^{6}$ time steps for populating the histogram for measurement of the velocity distribution.

The mean of the velocity distribution generated by the histograms of each slab is then assigned as the velocity associated with that slab, which is how each point on the velocity profiles shown in this section has been calculated. We have then non-dimensionalized the velocity profile thus generated using the basis set units. The temperature is calculated from the standard deviation of the velocity distributions generated by the histogram of each slab, as we expect a Maxwellian distribution of the velocities which entails a normal distribution of each of the velocity components with a standard deviation of $\sqrt{k_{B} T / m}$. We have then non-dimensionalized this temperature profile with the desired temperature $T_{0}$, which was an input to the simulations.

For sake of comparison, we have also performed similar simulations with a commercial CFD package COMSOL Multiphysics 5.0.[47] For a proper comparison, it is important that we must input the correct physical properties of our test fluid, particularly the viscosity of the fluid. So we performed independent bulk shear-flow simulations with our particle-based model based on the Lees-Edwards method[48], whereby we modify the periodic boundary conditions as follows. Assume that in a box of height $L_{y}$, the top wall moves with a velocity of $\dot{\gamma} L_{y} / 2$ in the x-direction and the bottom wall moves with a velocity of $-\dot{\gamma} L_{y} / 2$ in the $\mathrm{x}$-direction. When a particle crosses the bottom boundary and arrives at the top of the box, it is displaced by $\dot{\gamma} L t$ in the x-direction and the background velocity at the position of the particle is augmented by $\dot{\gamma} L$. Alternatively, if a particle crosses the top boundary and arrives at the bottom of the box, it is displaced by $-\dot{\gamma} L t$ in the $\mathrm{x}$-direction and the background velocity at the position of the particle is augmented by $-\dot{\gamma} L$. Moreover, during the force calculation, if a particle interacts with its neighbor across the boundary, then 
the background velocity of the neighboring particle is adjusted by $\pm \dot{\gamma} L$ depending on whether the neighbor is in the bottom or the top of the box. The viscosity from these simulations is then calculated as the ratio of the average stress $S_{x y}$ based on Eq.(2.26) and the shear rate $\dot{\gamma}$. This is the particle-contribution of the shear viscosity to which we must add the background fluid contribution, which we will discuss later. We fitted the flow curve thus obtained from these simulations with the Carreau equation[49] given in Eq.(3.36).

$$
\frac{\eta-\eta_{\infty}}{\eta_{0}-\eta_{\infty}}=\left[1+(\lambda \dot{\gamma})^{2}\right]^{\frac{n-1}{2}}
$$

where $\eta_{0}$ is the zero shear rate viscosity, $\eta_{\infty}$ is the infinite shear rate viscosity, $\lambda$ is a parameter with units of time and $n$ is a dimensionless parameter. The shear viscosity without the infinite shear rate viscosity component $\left(\eta-\eta_{\infty}\right)$ is fitted with the Carreau model optimizing the three parameters i.e. $\left(\eta_{0}-\eta_{\infty}\right), \lambda$ and $n$, is shown in Fig.2.5. Approximating the infinite shear rate viscosity $\eta_{\infty}$ as the background fluid viscosity, an estimate for $\eta_{\infty}$ can be obtained in terms of our model parameters as follows:

$$
\eta_{\infty}=\frac{R_{c}^{2} \rho^{\# \xi^{\prime}}}{28} .
$$

We have performed our flow simulations through a channel using a constant gravity field $g=F_{x} / m$, which must be translated to the analogous pressure drop per unit length of the channel for the corresponding COMSOL simulations. The relation between the pressure drop per unit length $\Delta P / L_{x}$ and our model parameters has been defined as follows:

$$
\Delta P / L_{x}=F_{x} \rho^{\#}
$$

Furthermore, we have assumed Stokes flow for the COMSOL simulations, thereby neglecting inertial terms from the Navier-Stokes equations. In Fig.2.6, we have shown the comparison of the results for the flow of our test system of a star polymer solution through a rectangular channel obtained from the simulations based on our model with the results from corresponding COMSOL simulations for a shear thinning fluid 
obeying the Carreau model. The three sub-figures a, b and c of Fig.2.6 show the results of the simulations performed for three different values of $\xi^{\prime}$ viz. 1, 2 and 5 respectively for a constant value of $\tau=3 \times 10^{-3} \sigma^{2} / D_{0}$ and maintaining a constant gravity field $g=F_{x} / m=133.4 D_{0}^{2} / \sigma^{3}$ by scaling forces $F_{x}$ proportional to $\xi^{\prime}$. Each of the sub-figures of Fig.6 shows the dimensionless density profile on the left, the dimensionless velocity profile in the center and the dimensionless temperature profile on the right.

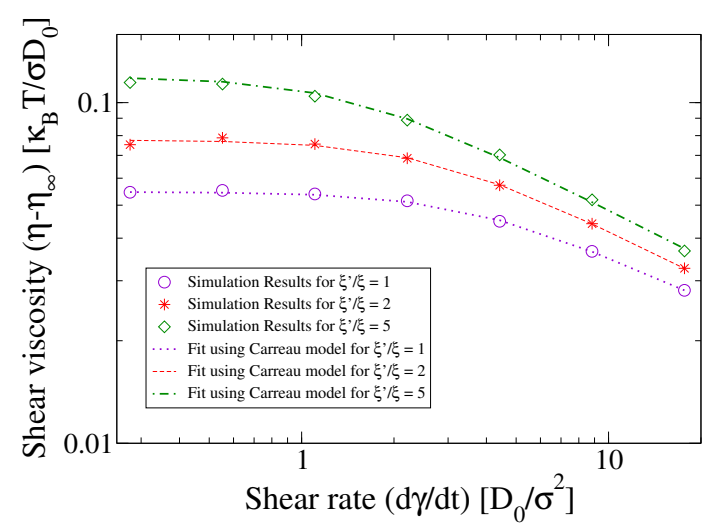

Figure 2.5: Flow curve obtained from shear flow simulations for different values of $\xi^{\prime} / \xi$ and $\tau=$ $3 \times 10^{-3} \sigma^{2} / D_{0}$ based on the Lees-Edwards method shown with the colored symbols and the fit using Carreau model shown with the colored dotted lines.

As can be seen from the Fig.2.6, the temperature is uniform throughout the crosssection and is close to its expected value that was set as input value $T_{0}$, which is the same value used for normalization. The density profiles correspond to the test system that we have employed. We see a layered fluid owing to the nature of the potential and the presence of a hard wall. This was to be expected looking at the radial distribution function from Fig.2.1 and the hard wall potential shown in Eq.2.18. The dimensionless velocity profiles agree satisfactorily with the profiles obtained from the COMSOL simulations for several parameter values. It is also interesting to note that despite the pronounced density fluctuations, we have obtained a rather smooth velocity profile. This is because the cut-offs for the normalized weight functions $w_{i j}$ 
and $f_{i j}$ used to calculate the average local properties and their gradients respectively have been chosen in such a way that we have about 15 particles within the cut-off, which effectively smooths out the fluctuations due to the density profile.
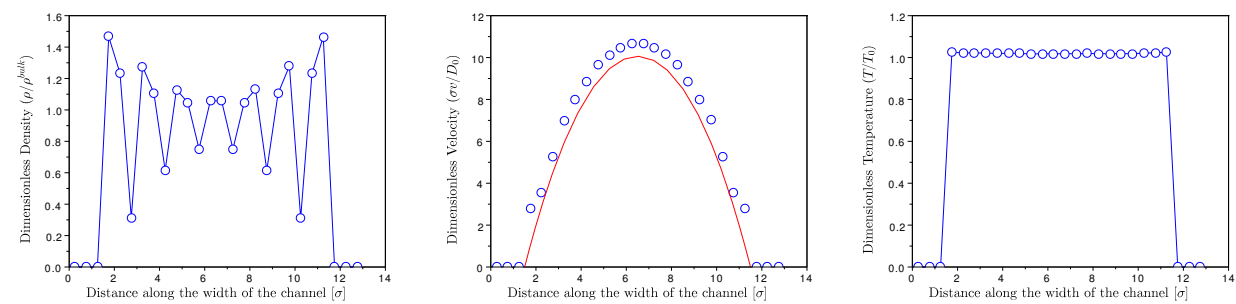

(a) $\xi^{\prime} / \xi=1$ for $\tau=3 \times 10^{-3} \sigma^{2} / D_{0}$.
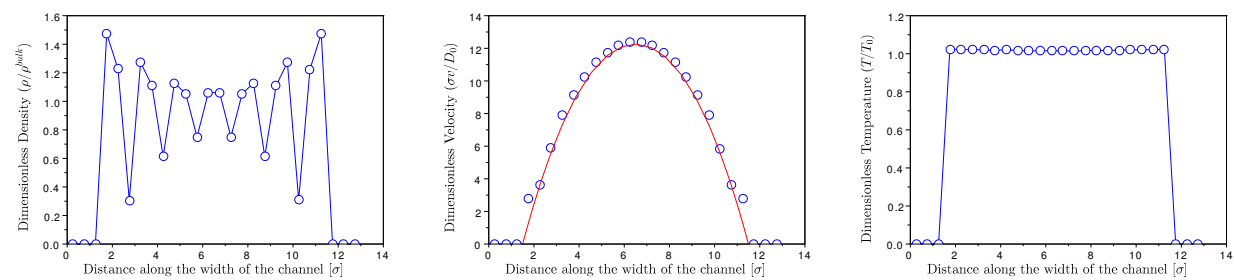

(b) $\xi^{\prime} / \xi=2$ for $\tau=3 \times 10^{-3} \sigma^{2} / D_{0}$.
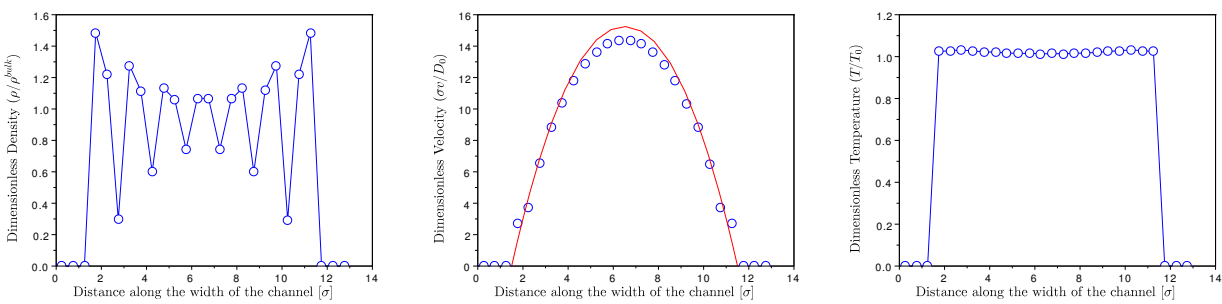

(c) $\xi^{\prime} / \xi=5$ for $\tau=3 \times 10^{-3} \sigma^{2} / D_{0}$.

Figure 2.6: Dimensionless density, velocity and temperature profiles for different parameter values using Morris boundary conditions. The velocity profile shown with circles is obtained from particle based simulations based on our model and the solid line shows the results from COMSOL simulations for a shear thinning fluid following Carreau equation.

It must be pointed out that the range of values for the parameters we have chosen are in such a way that the maximum particle Reynolds number is less than 1. An esti- 
mate for the particle Reynolds number can be obtained by solving the Navier-Stokes equation for a Newtonian fluid with a viscosity $v$ which is related to the parameters by Eq.(2.23). The maximum velocity in the channel of width $L_{y}$ for the flow of a Newtonian fluid with viscosity $v$ under the influence of a gravity field $g$ is as follows:

$$
v_{\max }=\frac{g L_{y}^{2}}{8 v} .
$$

Accordingly, the maximum particle Reynolds number is as follows:

$$
R e_{\text {max }}=\frac{\sigma g L_{y}^{2}}{8 v^{2}} .
$$

Using Eq.(2.38) and Eq.(2.23), we arrive at the following condition on the parameters:

$$
98 \frac{\sigma g \tau^{2}}{R_{c}^{2}}\left(\frac{L_{y}}{R_{c}}\right)^{2}<1
$$

In other words, we get an upper bound of $5.47 \times 10^{-3} \sigma^{2} / D_{0}$ for the value of $\tau$. Accordingly, we have used a value of $3 \times 10^{-3} \sigma^{2} / D_{0}$ for the channel flow simulations.

\subsection{Conclusions}

In this chapter, we have presented a technique for modeling flow of highly frictional soft matter systems in the presence of solid interfaces. The technique is largely based on a Galilean invariant coupling algorithm,[26] which we have modified and then thoroughly analyzed to further its understanding. We have demonstrated the relationship of the model parameters with the dynamic properties of the system. Furthermore, we have also presented an iterative procedure to theoretically predict the effect of the friction coefficients on the Mean-Squared-Displacement for a simple case of noninteracting particles, which can be used to then determine the values of the system parameters. We have presented the model in a very generalized manner so that it may be applied to a wide range of systems by tuning the parameters of the model.

It must however be pointed out that for highly viscous systems, the time-step required for the velocity propagator introduced by the Galilean invariant algorithm can 
be much smaller than the time-step required for the position updates based on Brownian dynamics. Thus, for such systems, it might be practical to use a multiscale approach where two different time-steps are used for the position and velocity updates. A large number of velocity updates can be carried out before the positions of the particles change significantly. Alternatively, a steady state solution may be calculated for the velocities every time the positions are updated.

Furthermore, it must be noted that although using the particles as the nodes, on which the background flow field is discretized, makes the model computationally efficient, the resolution of the background fluid is actually limited by the concentration of the particles in the system. For instance, in the case of a star polymer solution, the concentration of the polymers fixes the resolution of the background fluid because the particles play dual roles of representing the star polymers and also being the node points on which the background flow field is discretized. This may be resolved by having a model with two types of particles - ones representing the polymer particles and the others representing the background fluid, but for this case, correctly describing the interaction between the two types of particles is crucial.

\subsection{Appendix: Mean Squared Displacement of non-interacting particles}

In this section, we present the zeroth order calculation for mean squared displacement mentioned in the 'Discussion' sub-section of the section on 'Bulk flow simulations in quiescent state'. For the zeroth order calculation, we neglect the second term in the integrand on the right hand side of Eq.(2.31). Substituting the result into Eq.(2.29) and integrating, we get the displacement as:

$\Delta \mathbf{R}_{i}(t)=\mathbf{v}_{i}(0) \int_{0}^{t} \mathrm{~d} t^{\prime} e^{-t^{\prime} / \tau}+\int_{0}^{t} \mathrm{~d} t^{\prime} \int_{0}^{t^{\prime}} \mathrm{d} t^{\prime \prime} e^{-\left(t^{\prime}-t^{\prime \prime}\right) / \tau} \sum_{j=1, j \neq i}^{N} \frac{\mathrm{d} \mathbf{W}_{i j}^{v}\left(t^{\prime \prime}\right)}{\mathrm{d} t}+\int_{0}^{t} \mathrm{~d} t^{\prime} \frac{\mathrm{d} \mathbf{W}_{i}^{R}\left(t^{\prime}\right)}{\mathrm{d} t}$ 
Making use of $\int_{0}^{t} \mathrm{~d} t^{t^{\prime}} \int_{0}^{\prime} \mathrm{d} t^{\prime \prime}=\int_{0}^{t} \mathrm{~d} t^{\prime \prime} \int_{t^{\prime \prime}}^{t} \mathrm{~d} t^{\prime}$ and performing some integrations, we get:

$\Delta \mathbf{R}_{i}(t)=\tau \mathbf{v}_{i}(0)\left(1-e^{-t / \tau}\right)+\tau \int_{0}^{t} \mathrm{~d} t^{\prime}\left(1-e^{-\left(t-t^{\prime}\right) / \tau}\right) \sum_{j=1, j \neq i}^{N} \frac{\mathrm{d} \mathbf{W}_{i j}^{v}\left(t^{\prime}\right)}{\mathrm{d} t}+\int_{0}^{t} \mathrm{~d} t^{\prime} \frac{\mathrm{d} \mathbf{W}_{i}^{R}\left(t^{\prime}\right)}{\mathrm{d} t}$.

Squaring and averaging the above equation over the ensemble, and noting that the random variables have a zero mean and noting further that the random variables associated with the positions and velocities are uncorrelated with each other as defined in Eq.(2.12), we get:

$$
\begin{gathered}
\left\langle\Delta \mathbf{R}_{i}(t)^{2}\right\rangle=\tau^{2}\left(1-e^{-t / \tau}\right)^{2}\left\langle\mathbf{v}_{i}(0)^{2}\right\rangle+\tau^{2} \int_{0}^{t} \mathrm{~d} t^{\prime} \int_{0}^{t} \mathrm{~d} t^{\prime \prime}\left\{\left(1-e^{-\left(t-t^{\prime}\right) / \tau}\right)\left(1-e^{-\left(t-t^{\prime \prime}\right) / \tau}\right)\right. \\
\left.\sum_{j=1, j \neq i}^{N} \sum_{k=1, k \neq i}^{N}\left\langle\frac{\mathrm{d} \mathbf{W}_{i j}^{v}\left(t^{\prime}\right)}{\mathrm{d} t} \cdot \frac{\mathrm{d} \mathbf{W}_{i k}^{v}\left(t^{\prime \prime}\right)}{\mathrm{d} t}\right\rangle\right\}+\int_{0}^{t} \mathrm{~d} t^{\prime} \int_{0}^{t} \mathrm{~d} t^{\prime \prime}\left\langle\frac{\mathrm{d} \mathbf{W}_{i}^{R}\left(t^{\prime}\right)}{\mathrm{d} t} \cdot \frac{\mathrm{d} \mathbf{W}_{i}^{R}\left(t^{\prime \prime}\right)}{\mathrm{d} t}\right\rangle .
\end{gathered}
$$

In order to evaluate this equation further, we make use of :

$$
\begin{aligned}
\left\langle\mathbf{v}_{i}(0)^{2}\right\rangle & =\frac{3 k_{B} T}{m_{i}}, \\
\left\langle\frac{\mathrm{d} \mathbf{W}_{i j}^{v}\left(t^{\prime}\right)}{\mathrm{d} t} \cdot \frac{\mathrm{d} \mathbf{W}_{i k}^{v}\left(t^{\prime \prime}\right)}{\mathrm{d} t}\right\rangle & =\frac{6 k_{B} T}{m_{i}} \frac{f_{i j}}{\tau} \delta_{j k} \delta\left(t^{\prime}-t^{\prime \prime}\right), \\
\left\langle\frac{\mathrm{d} \mathbf{W}_{i}^{R}\left(t^{\prime}\right)}{\mathrm{d} t} \cdot \frac{\mathrm{d} \mathbf{W}_{i}^{R}\left(t^{\prime \prime}\right)}{\mathrm{d} t}\right\rangle & =\frac{6 k_{B} T}{\xi_{i}} \delta\left(t^{\prime}-t^{\prime \prime}\right) .
\end{aligned}
$$

Here we have used that fact the random terms at different times are uncorrelated, in which case $\delta_{t^{\prime} t^{\prime \prime}} / \mathrm{d} t=\delta\left(t^{\prime}-t^{\prime \prime}\right)$. Using the definition of mass given in Eq.(2.14), and simplifying, we obtain the expression for the mean squared displacement based on our zeroth iteration as follows:

$$
\left\langle\Delta \mathbf{R}_{i}(t)^{2}\right\rangle=\frac{6 k_{B} T}{\xi_{i}^{\prime}}\left[t-\tau\left(1-e^{-t / \tau}\right)\right]+\frac{6 k_{B} T}{\xi_{i}} t
$$

After a sufficiently long time i.e. $t \gg \tau$, we get:

$$
\left\langle\Delta \mathbf{R}_{i}(t)^{2}\right\rangle=\frac{6 k_{B} T}{\xi_{i}^{\prime}} t+\frac{6 k_{B} T}{\xi_{i}} t
$$




\section{Bibliography}

[1] D. C. Rapaport, The art of molecular dynamics simulation (Cambridge university press, 2004).

[2] M. P. Allen and D. J. Tildesley, Computer simulation of liquids (Oxford university press, 1989).

[3] G. A. Voth, Coarse-graining of condensed phase and biomolecular systems (CRC press, 2008).

[4] M. Karttunen, I. Vattulainen, and A. Lukkarinen, Novel methods in soft matter simulations, 640 (Springer Science \& Business Media, 2004).

[5] M. Murat and K. Kremer, The Journal of Chemical Physics 108, 4340 (1998).

[6] R. D. Groot and P. B. Warren, The Journal of Chemical Physics 107, 4423 (1997).

[7] C. N. Likos, H. Löwen, M. Watzlawek, B. Abbas, O. Jucknischke, J. Allgaier, and D. Richter, Physical Review Letters 80, 4450 (1998).

[8] A. Van den Noort, W. K. den Otter, and W. J. Briels, Europhysics Letters 80, 28003 (2007).

[9] I. S. Santos de Oliveira, A. van den Noort, J. T. Padding, W. K. den Otter, and W. J. Briels, The Journal of Chemical Physics 135, 104902 (2011).

[10] I. S. Santos de Oliveira, W. K. den Otter, and W. J. Briels, The Journal of Chemical Physics 137, 204908 (2012).

[11] P. J. Hoogerbrugge and J. M. V. A. Koelman, Europhysics Letters 19, 155 (1992).

[12] J. M. V. A. Koelman and P. J. Hoogerbrugge, Europhysics Letters 21, 363 (1993).

[13] P. Español and P. Warren, EPL (Europhysics Letters) 30, 191 (1995). 
[14] W. J. Briels, Theory of polymer dynamics (Lecture Notes, Uppsala, Sweden, 1994).

[15] C. W. Gardiner et al., Handbook of stochastic methods, Vol. 4 (Springer Berlin, 1985).

[16] E. Frey and K. Kroy, Annalen der Physik 14, 20 (2005).

[17] S. Izvekov and G. A. Voth, The Journal of Chemical Physics 125, 151101 (2006).

[18] C.-C. Huang, H. Xu, F. Crevel, J. Wittmer, and J.-P. Ryckaert, in Computer Simulations in Condensed Matter Systems: From Materials to Chemical Biology Volume 2 (Springer, 2006) pp. 379-418.

[19] I. S. Santos de Oliveira, B. W. Fitzgerald, W. K. den Otter, and W. J. Briels, The Journal of Chemical Physics 140, 104903 (2014).

[20] I. M. Ilie, W. K. den Otter, and W. J. Briels, The Journal of Chemical Physics 141, 065101 (2014).

[21] E. S. Boek, J. T. Padding, V. J. Anderson, W. J. Briels, and J. P. Crawshaw, Journal of Non-Newtonian Fluid Mechanics 146, 11 (2007).

[22] J. T. Padding, E. S. Boek, and W. J. Briels, The Journal of Chemical Physics 129, 074903 (2008).

[23] A. Van den Noort and W. J. Briels, Journal of Non-Newtonian Fluid Mechanics 152, 148 (2008).

[24] J. Sprakel, E. Spruijt, J. van der Gucht, J. T. Padding, and W. J. Briels, Soft Matter 5, 4748 (2009).

[25] J. Sprakel, J. T. Padding, and W. J. Briels, Europhysics Letters 93, 58003 (2011).

[26] J. T. Padding and W. J. Briels, The Journal of Chemical Physics 141, 244108 (2014). 
[27] R. A. Gingold and J. J. Monaghan, Monthly Notices of the Royal Astronomical Society 181, 375 (1977).

[28] L. B. Lucy, The Astronomical Journal 82, 1013 (1977).

[29] R. Gingold and J. Monaghan, Journal of Computational Physics 46, 429 (1982).

[30] J. J. Monaghan, Annual Review of Astronomy and Astrophysics 30, 543 (1992).

[31] J. J. Monaghan, Reports on Progress in Physics 68, 1703 (2005).

[32] P. Español and M. Revenga, Physical Review E 67, 026705 (2003).

[33] A. Vázquez-Quesada, M. Ellero, and P. Español, The Journal of Chemical Physics 130, 034901 (2009).

[34] S. Litvinov, M. Ellero, X. Hu, and N. A. Adams, Physical Review E 77, 066703 (2008).

[35] S. Litvinov, Q. Xie, X. Hu, N. Adams, and M. Ellero, Fluids 1, 7 (2016).

[36] X. Hu and N. Adams, Physics of Fluids 18, 101702 (2006).

[37] K. Müller, D. A. Fedosov, and G. Gompper, Journal of Computational Physics 281, 301 (2015).

[38] I. O. Götze, H. Noguchi, and G. Gompper, Physical Review E 76, 046705 (2007).

[39] J. P. Morris, P. J. Fox, and Y. Zhu, Journal of Computational Physics 136, 214 (1997).

[40] M. Revenga, I. Zuniga, and P. Español, Computer Physics Communications 121, 309 (1999).

[41] I. V. Pivkin and G. E. Karniadakis, Journal of Computational Physics 207, 114 (2005).

[42] J. Smiatek, M. P. Allen, and F. Schmid, The European Physical Journal E 26, 115 (2008). 
[43] L. Brookshaw, in Proceedings of the Astronomical Society of Australia, Vol. 6 (1985) pp. 207-210.

[44] D. Vlassopoulos and G. Fytas, in High Solid Dispersions (Springer, 2009) pp. $1-54$.

[45] M. Daoud and J. Cotton, Journal de Physique 43, 531 (1982).

[46] J. T. Padding, E. Van Ruymbeke, D. Vlassopoulos, and W. J. Briels, Rheologica Acta 49, 473 (2010).

[47] Comsol, Multiphysics User Guide for COMSOL 5.0 (2014).

[48] A. Lees and S. Edwards, Journal of Physics C: Solid State Physics 5, 1921 (1972).

[49] R. Bird, W. Stewart, and E. Lightfoot, Transport Phenomena, Wiley International edition (Wiley, 2007). 


\section{Two-way coupling of polymers with explicit solvent}

We present a novel coarse-grain particle-based simulation technique for modeling self-developing flow of dilute and semi-dilute polymer solutions. The central idea in this chapter is the two-way coupling between a mesoscopic polymer model and a phenomenological fluid model. As our polymer model, we choose Responsive Particle Dynamics (RaPiD), a Brownian Dynamics method, which formulates the so-called 'conservative' and 'transient' pair-potentials through which the polymers interact besides experiencing random forces in accordance with the fluctuation dissipation theorem. In addition to these interactions, our polymer blobs are also influenced by the background solvent velocity field, which we calculate by solving the Navier-Stokes equation discretized on a moving grid of fluid blobs using the Smoothed Particle Hydrodynamics $(\mathrm{SPH})$ technique. While the polymers experience this frictional force opposing their motion relative to the background flow field, our fluid blobs also in turn are influenced by the motion of the polymers, through an interaction term. This makes our technique a two-way coupling algorithm. We have constructed this interaction term in such a way that momentum is conserved locally, thereby preserving long range hydrodynamics. Furthermore, we have derived pairwise fluctuation terms for the velocities of the fluid blobs using the Fokker-Planck equation, which have been alternatively derived using the General Equation for the Non-Equilibrium ReversibleIrreversible Coupling (GENERIC) approach in Smoothed Dissipative Particle Dynamics (SDPD) literature. We have applied our technique to study the rheology of two different concentrations of our model linear polymer solutions. The results show that the polymers and the fluid are coupled very well with each other, showing no lag between their velocities. Furthermore, our results show non-Newtonian shear thinning and the characteristic flattening of the Poiseuille flow profile typically observed for polymer solutions. ${ }^{1}$

${ }^{1}$ This chapter has been published as V. R. Ahuja, J. van der Gucht, and W. J. Briels, J. Chem. Phys, 148(3), 034902 (2018) 


\subsection{Introduction}

Simulating the dynamics of polymers in solution has been a subject of continuous interest for almost three decades now, yet a final resolution of the problem eludes us owing to its inherent complexity. This problem poses many fundamental questions such as - how do these large polymer molecules interact with the solvent fluid molecules and how do they exchange momentum under flowing conditions? Given the enormous size of the polymer molecules dissolved in a fluid made up of molecules which are orders of magnitude smaller, it is practically impossible to even simulate just tens of polymer molecules together with all the millions of fluid molecules in their vicinity if all of them are to be resolved down to the atomic level. Hence, coarse-graining is an inherent part of simulating polymers in solution.

The obvious question that arises next is what should be the level of coarse-graining, i.e. how well do we resolve the polymer molecules and the fluid? Very broadly speaking, one can categorize the simulation techniques into two major branches - particlebased simulations and Computational Fluid Dynamics (CFD) simulations using a continuum approach. Since there is a plethora of techniques under each category, we restrict the discussion here to particle-based simulation techniques, which, we might add, have the advantage of being able to link macroscopic flow behavior to microscopic interactions. Among the particle-based simulation techniques that have been used over the years to model the dynamics of polymers in solution, there is again an enormous variety of methods employing different levels of coarse-graining for the polymers and the fluid. There have been studies employing molecular dynamics (MD) for simulating polymers modeled as a chain of a few 'monomers' connected to each other with springs in a solvent represented by a few thousand fluid particles treated as soft spheres.[1, 2] Hybrid models have been developed which combine two different levels of coarse-graining for the polymers and the fluid. One such hybrid model combines the MD approach for the polymer chain with the Lattice Boltzmann (LB) approach for the solvent.[3] Other hybrid models combine the MD approach for the polymers with the multi-particle collision dynamics (MPCD) or Stochastic 
Rotation Dynamics (SRD) method for the solvent.[4, 5, 6, 7, 8, 9, 10] Mesoscopic methods like Dissipative Particle Dynamics (DPD)[11, 12, 13] have also been employed to study polymer solutions by representing the solvent with DPD particles acting as 'fluid elements' and representing the polymer molecules by a chain of such DPD particles connected to each other with springs.[14, 15, 16, 17, 18, 19, 20, 21, 22] More recently, a technique called Smoothed Dissipative Particle Dynamics (SDPD) has been developed, [23, 24] which is a modified version of Smoothed Particle Hydrodynamics (SPH) [25, 26, 27, 28, 29] with added DPD-like pairwise thermal fluctuation terms. This technique has also been used for simulating polymer solutions in a similar way like DPD.[30, 31, 32]

Furthermore, there are other simulation techniques on a much higher level of coarse-graining based on Brownian Dynamics such as Responsive Particle Dynamics (RaPiD),[33, 34] in which the solvent is not explicitly modeled but is rather implicitly present and the polymer molecules are represented by their centres-of-mass and interact through pair-potentials. RaPiD has been used for studying various phenomena related to polymer solutions.[35, 36, 37] Recently, another technique based on Brownian Dynamics with an implicit background fluid having hydrodynamic interactions was developed for modeling self-developing flow of polymer solutions in the bulk as well as in the presence of solid interfaces.[38, 39] As the background fluid is implicitly present at the positions of the polymer molecules, this technique is computationally efficient as it does not need separate position updates for the fluid particles. Nevertheless, the resolution of the background fluid is fixed by the concentration of the polymers and one is not free to choose an arbitrary equation of state for the fluid. To resolve these problems, it was envisaged that two different particles be used - one for modeling polymers and the other for the fluid. However, this can only be done if a proper interaction term is constructed that not only couples the motion of the polymers and the fluid but also conserves momentum so as to preserve long range hydrodynamics. This is precisely what we present in this chapter. Furthermore, we have derived pairwise fluctuation terms for the fluid using the Fokker Planck equation so that the steady state probability distributions of the positions and velocities 
of the fluid in a quiescent state correspond to the equilibrium distribution. We arrive essentially at the same result that has been derived using the General Equation for the Non-Equilibrium Reversible-Irreversible Coupling (GENERIC) approach for an incompressible SDPD fluid.[23, 24] However, these fluctuation terms may be neglected if they do not play a major role in the case being studied, under which circumstance the fluid model reduces to standard SPH.

\subsection{Model development}

\subsubsection{Equation of motion for the polymer blobs}

Consider the motion of a mesoscopic polymer blob $a$ dissolved in a solvent, alternatively referred to as the background fluid in this work, under flowing conditions. It is well known that for overdamped systems, the timescale over which the particles move to any significant extent is orders of magnitude larger than the timescale over which their velocities get completely thermalized. Therefore, these velocities may be integrated out and the positions of the particles can be updated using a first order Brownian Dynamics propagator:[40, 41]

$$
\mathrm{d} \mathbf{r}_{a}=\mathbf{v}\left(\mathbf{r}_{a}\right) \mathrm{d} t+\left(\frac{\mathbf{F}_{a}}{\xi_{a}}\right) \mathrm{d} t+k_{B} T \frac{\partial}{\partial \mathbf{r}_{a}}\left(\frac{1}{\xi_{a}}\right) \mathrm{d} t+\mathrm{d} \mathbf{W}_{a}^{R}
$$

Here, $\mathbf{r}_{a}$, shorthand for $\mathbf{r}_{a}(t)$, is the position of polymer blob $a$ at time $t$ and $\mathrm{d} t$ is the time-step. We would like to emphasize that $\mathbf{v}\left(\mathbf{r}_{a}\right)$, shorthand for $\mathbf{v}\left(\mathbf{r}_{a}, t\right)$, is not the velocity of the polymer blob $a$ but rather the background fluid velocity at the position of polymer blob $a$ at time $t$. $\mathbf{F}_{a}$, shorthand for $\mathbf{F}_{a}(t)$, is the driving force acting on polymer blob $a$ as a result of the interaction with other polymer blobs, in addition to any force field that may have been applied. In the remaining part of the chapter, we will not include the $t$ in our notation, tacitly assuming that it is implicitly present. $\xi_{a}$, shorthand for $\xi\left(\mathbf{r}_{a}\right)$, is the friction coefficient at the position of polymer blob $a$. The third term on the right hand side of eq.(3.1) is a drift term accounting for the spatial variation of the friction coefficient, which we have neglected in this study as we have assumed a constant friction coefficient for the sake of simplicity. The last term on the 
right hand side of eq.(3.1), i.e. $\mathrm{dW}_{a}^{R}$, is a random displacement typical of Brownian dynamics simulations. This random displacement is uncorrelated in time and has a magnitude that is calculated in accordance with the fluctuation dissipation theorem, satisfying:

$$
\left\langle\mathrm{d}^{R} \mathrm{~d}_{a}^{R}\right\rangle=2 k_{B} T\left(\frac{\mathrm{d} t}{\xi_{a}}\right) \delta_{a b} \mathbf{I} .
$$

Since the background flow field is typically not known a priori, it must be calculated during the simulation in real time. We calculate this background velocity field on a moving grid of fluid blobs which serve as the node points as in a Smoothed Particle Hydrodynamics (SPH) simulation.[25, 26, 27, 28, 29]. Since the flow field is calculated only at a finite number of node points, the background fluid velocity at the position of the polymer blob $a$ in eq.(3.1) must be estimated based on interpolation. To this end, we use the integral interpolant:

$$
\mathbf{v}\left(\mathbf{r}_{a}\right)=\int w^{f}\left(\left|\mathbf{r}-\mathbf{r}_{a}\right|\right) \mathbf{v}(\mathbf{r}) \mathrm{d}^{3} r
$$

where $\mathrm{d}^{3} r$ is a volume element and $w^{f}(r)$ is a normalized weight function with a cutoff $R_{c}$ satisfying for a 3-D simulation: $\int_{0}^{R_{c}} 4 \pi r^{2} w^{f}(r) \mathrm{d} r=1$. The superscript $f$ in $w^{f}(r)$ indicates that this is the weight function used for the fluid blobs. The integral in eq.(3.3) can be replaced by a summation running over each of the fluid neighbors $i$ of polymer blob $a$ using the standard SPH formulation:

$$
\mathbf{v}\left(\mathbf{r}_{a}\right)=\sum_{i=1}^{N_{f}} \frac{w^{f}\left(r_{a i}\right)}{n_{i}^{f}} \mathbf{v}_{i}
$$

Here, $N_{f}$ is the total number of fluid blobs and $r_{a i}$, shorthand for $\left|\mathbf{r}_{a}-\mathbf{r}_{i}\right|$, is the distance between polymer blob $a$ and fluid blob $i$ at time $t . n_{i}^{f}$ is the local number density of fluid blobs at the position of the fluid blob $i$ at time $t$, which is calculated by running over each of the fluid neighbors $j$ of the fluid blob $i$ using the aforementioned weight function $w^{f}(r)$, as shown below:

$$
n_{i}^{f}=\sum_{j=1}^{N_{f}} w^{f}\left(r_{i j}\right)
$$


For calculating the driving force $F_{a}$ in the second term of eq.(3.1), we resort to the Brownian dynamics based polymer model RaPiD, [33] in which the driving force is calculated as the gradient of a two-part pair-potential:

$$
\mathbf{F}_{a}=-\frac{\partial}{\partial \mathbf{r}_{a}}\left[\Phi_{c}+\Phi_{t}\right]
$$

where $\Phi_{c}$ is the conservative potential and $\Phi_{t}$ is the transient potential, both of which shall be defined and described in section 3.3.

It is interesting to note that by rearranging eq.(3.1) and using eq.(3.5), and assuming a constant friction coefficient $\xi$ for the sake of simplicity, one may obtain the effective frictional force $\mathbf{F}_{a}^{p f}$ acting on the polymer blob $a$ at time $t$, opposing its motion relative to the background flow field, as:

$$
\mathbf{F}_{a}^{p f}=-\xi \sum_{i=1}^{N_{f}} \frac{w^{f}\left(r_{a i}\right)}{n_{i}^{f}}\left(\frac{\mathrm{d} \mathbf{r}_{a}^{*}}{\mathrm{~d} t}-\mathbf{v}_{i}\right)
$$

Here, $\mathrm{d} \mathbf{r}_{a}^{*} / \mathrm{d} t$ is the deterministic part of the rate of change of the position of the polymer blob $a$, i.e. as calculated without the random fluctuation $\mathrm{d} \mathbf{W}_{a}^{R}$ and $\mathbf{v}_{i}$ is the velocity of the fluid blob $i$ at time $t$. Furthermore, it can be said that it is through this frictional force that we essentially couple the motion of the polymer blobs with the motion of the background fluid. In the following subsection, we shall refer to this frictional force to motivate the interaction force acting on the fluid blobs due to the polymer blobs.

\subsubsection{Equation of motion for the fluid blobs}

For calculating the background flow field, we use the incompressible Navier-Stokes equation for Fluctuating Hydrodynamics, [42, 43, 44, 45] augmented with an additional interaction term representing the force acting on the fluid due to the polymer:

$$
\frac{\mathrm{D} \mathbf{v}}{\mathrm{D} t}(\mathbf{r})=-\left(\frac{\nabla P}{\rho}\right)(\mathbf{r})+\eta\left(\frac{\nabla^{2} \mathbf{v}}{\rho}\right)(\mathbf{r})+\mathbf{g}(\mathbf{r})+\mathbf{F}^{f p}(\mathbf{r})+\mathrm{d}^{v}(\mathbf{r})
$$

Here, the term on the left hand side is the material derivative of the velocity $\mathbf{v}(\mathbf{r}), \rho$ is the mass density of the fluid, $\eta$ is the viscosity of the fluid, $\nabla P(\mathbf{r})$ is the pressure 
gradient, $\mathbf{g}(\mathbf{r})$ is the acceleration due to body forces, $\mathbf{F}^{f p}(\mathbf{r})$ is the interaction term which represents the force on the fluid due to the polymer and $\mathrm{dW}^{v}(\mathbf{r})$ is a random fluctuation term.

Now, instead of solving the above equation on a Eulerian grid as in CFD, we discretize the above equation on a moving grid of fluid blobs as in SPH.[25, 26, 27 , 28, 29] Since the fluid blobs themselves move with the flow field, the position of a fluid blob $i$ can be updated using:

$$
\mathrm{d} \mathbf{r}_{i}=\mathbf{v}_{i} \mathrm{~d} t
$$

Here, once again we clarify that $\mathbf{r}_{i}$ is the position of fluid blob $i$ at time $t$ and $\mathbf{v}_{i}$ is the velocity of that fluid blob at that time. As a direct consequence of this, the material derivative of the velocity can be readily calculated as the rate at which the velocity of the fluid blobs change in the Lagrangian frame of reference, as shown below:

$$
\frac{\mathrm{D} \mathbf{v}}{\mathrm{D} t}\left(\mathbf{r}_{i}\right)=\frac{\mathrm{d} \mathbf{v}_{i}}{\mathrm{~d} t}
$$

and $\mathbf{g}(\mathbf{r})$ at the position of fluid blob $i$ can be discretized as $\mathbf{g}_{i}$. For calculating the pressure gradient and the viscous dissipation term, standard SPH finite-difference like forms[29] have been employed:

$$
\begin{aligned}
\left(\frac{\nabla P}{\rho}\right)\left(\mathbf{r}_{i}\right) & =\frac{1}{m} \sum_{j=1}^{N_{f}}\left(\frac{P_{i}}{\left(n_{i}^{f}\right)^{2}}+\frac{P_{j}}{\left(n_{j}^{f}\right)^{2}}\right) \frac{\mathrm{d} w^{f}}{\mathrm{~d} r}\left(r_{i j}\right) \frac{\mathbf{r}_{i j}}{r_{i j}}, \\
\left(\frac{\boldsymbol{\nabla}^{2} \mathbf{v}}{\rho}\right)\left(\mathbf{r}_{i}\right) & =\frac{1}{m} \sum_{j=1}^{N_{f}}\left(\frac{2}{n_{i}^{f} n_{j}^{f}}\right) \frac{\mathrm{d} w^{f}}{\mathrm{~d} r}\left(r_{i j}\right) \frac{\mathbf{v}_{i j}}{r_{i j}},
\end{aligned}
$$

where $\mathbf{v}_{i j}$ is the velocity of fluid blob $i$ relative to fluid blob $j$ and $m$ is the mass of the fluid blobs, which is calculated as the ratio of the mass density of the fluid $\rho$ to the average number density of the fluid blobs $\bar{n}^{f}$, i.e. $m=\rho / \bar{n}^{f}$.

$\mathbf{F}^{f p}(\mathbf{r})$ discretized at the position of the fluid blob $i$ is denoted by $\mathbf{F}_{i}^{f p}$, which represents the influence of the surrounding polymer blobs on the fluid blob $i$ at time $t$. We may now refer to the frictional force $\mathbf{F}_{a}^{p f}$ shown in eq.(3.7) and construct the interaction term $\mathbf{F}_{i}^{f p}$, such that for each individual polymer-fluid pair of polymer blob $a$ and fluid blob $i$, the force exerted by polymer blob $a$ on fluid blob $i$ is equal 
and opposite to the frictional force exerted by fluid blob $i$ on polymer blob $a$. Thus, the total force on fluid blob $i$, calculated by summing over the forces due to all the individual polymer blobs, would then be:

$$
\mathbf{F}_{i}^{f p}=\xi \sum_{a=1}^{N_{p}} \frac{w^{f}\left(r_{a i}\right)}{n_{i}^{f}}\left(\frac{\mathrm{d} \mathbf{r}_{a}^{*}}{\mathrm{~d} t}-\mathbf{v}_{i}\right)
$$

where $N_{p}$ is the total number of polymer blobs. However, due to computational reasons, which shall be explained at the end of this subsection, we adopt a slightly modified version for the interaction term:

$$
\mathbf{F}_{i}^{f p}=\xi \sum_{a=1}^{N_{p}} \frac{w^{f}\left(r_{a i}\right)}{n_{i}^{f}}\left(\frac{\mathrm{d} \mathbf{r}_{a}^{*}}{\mathrm{~d} t}-\mathbf{v}\left(\mathbf{r}_{a}\right)\right)
$$

where $\mathbf{v}\left(\mathbf{r}_{a}\right)$ is the velocity of the fluid interpolated at the position of polymer blob $a$ at time $t$, as defined in the previous subsection in eq.(3.4). Although this means that we do not achieve pairwise momentum conservation for the polymer-fluid interactions, we still achieve momentum conservation on a local level in such a way that the total frictional force exerted on any given polymer blob $a$ by the fluid is distributed back in its entirety to the fluid blobs in its local vicinity, the proof of which has been provided in appendix 3.6.1. We can further simplify $\mathbf{F}_{i}^{f p}$ using eq.(3.1) and eq.(3.14), to arrive at:

$$
\mathbf{F}_{i}^{f p}=\sum_{a=1}^{N_{p}} \frac{w^{f}\left(r_{a i}\right)}{n_{i}^{f}} \mathbf{F}_{a}
$$

We must emphasize that it is through this term that the fluid blobs experience the influence of the polymer blobs. Thus, the two interaction terms $\mathbf{F}^{p f}$ and $\mathbf{F}^{f p}$ essentially make this model a two-way coupling scheme. We might add that this strategy of redistributing the force is reminiscent of a two-way coupling model that was developed for gas-fluidized beds.[46]

Combining the results from equations (3.8), (3.10), (3.11), (3.12) and (3.15), and discretizing the fluctuation term $\mathrm{d}^{v}(\mathbf{r})$ at the position of the fluid blob $i$ as a pairwise sum of random terms denoted by $\mathrm{d} \mathbf{W}_{i j}^{v}$, we arrive at the following equation that we 
have used in our simulations to update the velocities of the fluid blobs:

$$
\begin{aligned}
\mathrm{d} \mathbf{v}_{i}= & -\frac{\mathrm{d} t}{m}\left[\sum_{j=1}^{N_{f}}\left(\frac{P_{i}}{\left(n_{i}^{f}\right)^{2}}+\frac{P_{j}}{\left(n_{j}^{f}\right)^{2}}\right) \frac{\mathrm{d} w^{f}}{\mathrm{~d} r}\left(r_{i j}\right) \frac{\mathbf{r}_{i j}}{r_{i j}}+\sum_{j=1}^{N_{f}} f_{i j} \mathbf{v}_{i j}\right]+\mathbf{g}_{i} \mathrm{~d} t \\
& +\frac{\mathrm{d} t}{m}\left[\sum_{a=1}^{N_{p}} \frac{w^{f}\left(r_{a i}\right)}{n_{i}^{f}} \mathbf{F}_{a}\right]+\sum_{j=1}^{N_{f}} \mathrm{~d} \mathbf{W}_{i j}^{v},
\end{aligned}
$$

where $f_{i j}$, shorthand for $f\left(r_{i j}\right)$, is a symmetric function defined as follows:

$$
f\left(r_{i j}\right)= \begin{cases}-\left(\frac{2 \eta}{n_{i}^{f} n_{j}^{f}}\right) \frac{1}{r_{i j}} \frac{\mathrm{d} w^{f}}{\mathrm{~d} r}\left(r_{i j}\right) & \text { for } r_{i j} \leq R_{c} \\ 0 & \text { for } r_{i j} \geq R_{c} .\end{cases}
$$

The pairwise velocity fluctuation terms are uncorrelated in time and have been calculated in an anti-symmetric manner such that $\mathrm{d} \mathbf{W}_{i j}^{v}=-\mathrm{d} \mathbf{W}_{j i}^{v}$, so that the velocity updates are momentum conserving. The properties of these momentum fluctuations $\mathrm{d} \mathbf{W}_{i j}$ have been calculated in a way that the steady state probability distribution of the positions and velocities of the fluid in a quiescent state yields the expected equilibrium distribution. From a detailed derivation shown in the appendix 3.6.2, we have:

$$
\begin{aligned}
\left\langle\mathrm{d} \mathbf{W}_{i j}^{v} \mathrm{~d} \mathbf{W}_{i j}^{v}\right\rangle & =\left(\frac{2 k_{B} T}{m}\right)\left(\frac{\mathrm{d} t}{m}\right) f_{i j} \mathbf{I}, \\
\left\langle\mathrm{d}^{v} \mathbf{W}_{i k}^{v} \mathbf{d}_{j l}^{v}\right\rangle & =\mathbf{0} \quad(i k \neq j l \wedge i k \neq l j) .
\end{aligned}
$$

Notice that if instead of eq.(3.14), a pairwise anti-symmetric interaction term as shown in eq.(3.13) would have been used, then we would have ended up with an additional term proportional to the product of the time-step $\mathrm{d} t$ and the friction coefficient $\xi$ in the equation of motion for the fluid blobs. This would deprive us of the whole advantage of using Brownian Dynamics for the polymers which allows us to use a larger time-step for overdamped systems where the friction coefficient $\xi$ is large. This is the reason for not using eq.(3.13) as the interaction term for the fluid blobs.

It is also interesting to note that if we would not have neglected the spatial variation of the friction coefficient in eq.(3.1), then we would have had an additional term proportional to $\frac{\partial}{\partial \mathbf{r}_{a}} \ln \left(\xi_{a}\right)$ in the equation of motion for the fluid blobs. However, 
since this term grows only weakly with increasing $\xi_{a}$, it would not affect our proposed technique significantly.

\subsection{Test System and Parameters}

\subsubsection{The conservative potential}

We have tested our technique with a model linear polymer solution at different concentrations. We use the Flory-Huggins potential, which has been used in the literature for describing the conservative part of the interaction potential between the linear polymers[35, 36, 37], defined as follows:

$$
\Phi_{c}=p k_{B} T \sum_{a=1}^{N_{p}}\left[\left(\frac{1-\phi_{a}}{\phi_{a}}\right) \ln \left(1-\phi_{a}\right)-\chi \phi_{a}\right] .
$$

Here, $N_{p}$ is the total number of polymer blobs in solution as mentioned before, $\Phi_{c}$ is the conservative potential, $p$ is the number of Kuhn segments in a polymer blob, $\chi$ is the solvent interaction parameter and $\phi_{a}$ is the local volume fraction of polymer blobs in the neighborhood of polymer blob $a$ at time $t$ calculated as:

$$
\phi_{a}=\frac{n_{a}^{p}}{n_{\max }^{p}}
$$

Here, $n_{\max }^{p}$ is the maximum number density of polymers that the system is allowed to reach, i.e. the melt density and $n_{a}^{p}$ is the local number density of polymer blobs at the position of the polymer blob $a$ calculated as:

$$
n_{a}^{p}=\sum_{b=1}^{N_{p}} w^{p}\left(r_{a b}\right)
$$

where $r_{a b}$ is the distance between polymers $a$ and $b$ at time $t$ and $w^{p}(r)$ is a normalized weight function with a cut-off $r_{c}$ satisfying for a 3-D simulation: $\int_{0}^{r_{c}} 4 \pi r^{2} w^{p}(r) \mathrm{d} r=$ 1. The superscript $p$ in $w^{p}(r)$ indicates that this is the weight function used for the polymer blobs. 


\subsubsection{The transient potential}

We have used the RaPiD technique to incorporate memory effects into the simulation model through a transient potential, which depends on the history of the interacting polymers by keeping track of dynamic variables, given by:[33, 34]

$$
\Phi_{t}=\frac{1}{2} \alpha \sum_{a, b=1}^{N_{p}}\left(\lambda_{a b}-\lambda_{a b}^{e q}\right)^{2} .
$$

Here, $\Phi_{t}$ the transient potential, $\alpha$ is a parameter associated with the strength of the interactions or in other words the penalty for the deviation of the dynamic variable $\lambda_{a b}$ from its equilibrium value $\lambda_{a b}^{e q}$. The variable $\lambda_{a b}$, shorthand for $\lambda_{a b}(t)$, is a dimensionless dynamic variable representing the degree of intermixing of the polymers $a$ and $b$, which evolves over time based on the following first order stochastic differential equation:

$$
\mathrm{d} \lambda_{a b}=-\left(\lambda_{a b}-\lambda_{a b}^{e q}\right) \frac{\mathrm{d} t}{\tau}+\mathrm{d} \mathbf{W}_{a b}^{\lambda}
$$

where $\tau$ is the relaxation time and $\mathbf{W}_{a b}^{\lambda}$ is a Wiener process with time-uncorrelated increments satisfying:

$$
\left\langle\mathrm{d} \mathbf{W}_{a b}^{\lambda} \mathrm{d} \mathbf{W}_{a b}^{\lambda}\right\rangle=\left(\frac{2 k_{B} T}{\alpha}\right)\left(\frac{\mathrm{d} t}{\tau}\right) \mathbf{I} .
$$

The variable $\lambda_{a b}^{e q}$, shorthand for $\lambda^{e q}\left(r_{a b}\right)$, is the equilibrium value of the variable $\lambda_{a b}$ for the distance $r_{a b}$, shorthand for $r_{a b}(t)$, between polymers $a$ and $b$ at time $t$. For the definition of $\lambda_{a b}^{e q}$, we use here the following form that has been used in the literature: $[35,36,37]$

$$
\lambda^{e q}\left(r_{a b}\right)= \begin{cases}\left(1-\frac{r_{a b}}{r_{c}}\right)^{2} & \text { for } r_{a b} \leq r_{c} \\ 0 & \text { for } r_{a b} \geq r_{c} .\end{cases}
$$

\subsubsection{The equation of state}

For the fluid, we use the commonly used pseudo-incompressible equation of state, which is essentially a modified version of the Tait equation of state with the exponent 
chosen as 7 so that it works well for water:[47, 48]

$$
P_{i}=P_{0}\left[\left(\frac{n_{i}^{f}}{\bar{n}^{f}}\right)^{7}-1\right],
$$

where $P_{0}$ is chosen such that the velocity of sound in the simulation is sufficiently large in order that the density fluctuations are sufficiently small, which results in a fluid that resembles an incompressible fluid.

\subsubsection{Definition of weight functions and system parameters}

For the polymer blobs, we have used a normalized weight function that has been used earlier in literature, where the polymer model RaPiD has been employed[35, 36, 37]. It is a monotonically decreasing function with derivatives upto the first order continuous to prevent any discontinuities in the forces which are related to the first derivative of the weight function. Furthermore, it has a non-zero derivative at the origin in order to have a net repulsive force at zero-distance to prevent clustering. It is given by $w^{p}(r)$ :

$$
w^{p}(r)= \begin{cases}\frac{15}{2 \pi\left(r_{c}^{5}-\sigma^{5}\right)}\left(r_{c}-\sigma\right)\left(r_{c}+\sigma-2 r\right) & \text { for } r \leq \sigma \\ \frac{15}{2 \pi\left(r_{c}^{5}-\sigma^{5}\right)}\left(r-r_{c}\right)^{2} & \text { for } \sigma \leq r \leq r_{c} \\ 0 & \text { for } r \geq r_{c},\end{cases}
$$

where the cut-off $r_{c}$ is chosen as $2.5 \sigma$. It can be easily checked that the weight function $w^{p}(r)$ is normalized in 3-dimensions within this cut-off radius $r_{c}$ such that $\int_{0}^{r_{c}} 4 \pi r^{2} w^{p}(r) d r=1$.

For the fluid blobs, we have chosen the normalized $M_{4}$ kernel commonly used in $\mathrm{SPH}[29]$ as the weight function. It is a cubic spline (piecewise continuous polynomial of degree 3 ) having derivatives up to the second order continuous and a cut-off of $R_{c}=2 h$. It is given by $w^{f}(r)$ :

$$
w^{f}(r)= \begin{cases}\frac{1}{4 \pi h^{3}}\left[\left(2-\frac{r}{h}\right)^{3}-4\left(1-\frac{r}{h}\right)^{3}\right] & \text { for } r \leq h \\ \frac{1}{4 \pi h^{3}}\left(2-\frac{r}{h}\right)^{3} & \text { for } h \leq r \leq 2 h \\ 0 & \text { for } r \geq 2 h\end{cases}
$$


where $h$ is what is commonly referred to as the support of the weight function. Again, it can be easily checked that the weight function $w^{f}(r)$ is normalized in 3-dimensions within the cut-off radius such that $\int_{0}^{R_{c}} 4 \pi r^{2} w^{f}(r) d r=1$.

Table 3.1: Summary of system parameters

\begin{tabular}{llll}
\hline \hline System parameter & Symbol & Value & Unit \\
\hline Length scale & $\sigma$ & 5.0 & $\mu \mathrm{m}$ \\
Time step & $\mathrm{d} t$ & 1.0 & $\mu \mathrm{s}$ \\
Temperature & $T$ & 300 & $\mathrm{~K}$ \\
Density of fluid & $\rho$ & 1000 & $\mathrm{~kg} / \mathrm{m}^{3}$ \\
Viscosity of fluid & $\eta$ & 1.0 & $\mathrm{mPa} . \mathrm{s}$ \\
Resolution of fluid & $\bar{n}^{f}$ & $1.9099 \times 10^{15}$ & $\mathrm{particles} / \mathrm{m}^{3}$ \\
Pressure coefficient & $P_{0}$ & 0.13 & $\mathrm{~Pa}$ \\
Friction coefficient & $\xi$ & $1.0 \times 10^{-7}$ & $\mathrm{~kg} / \mathrm{s}$ \\
Strength of polymer interactions & $\alpha$ & 500 & $k_{B} T$ \\
Relaxation time & $\tau$ & 1.0 & $\mathrm{~s}$ \\
Number of Kuhn segments & $\mathrm{p}$ & 300,000 & - \\
Maximum number density of polymers & $n_{\max }^{p}$ & $1.0 \times 10^{4}$ & $\mathrm{C}^{*}$ \\
Flory Huggins interaction parameter & $\chi$ & 0.5 & - \\
\hline \hline
\end{tabular}

We have chosen $h=2 \sigma$ for our simulations such that the cutoff radius $R_{c}$ for the fluid blobs is larger than the cut-off radius $r_{c}$ chosen for the polymer blobs because the weight function $w^{f}(r)$ must be able to accurately estimate the second order derivatives occurring in the equation of motion for the fluid blobs. Following the same logic, it immediately follows that for the polymer-fluid interactions, as we do not need to calculate any gradients, we can use the weight function $w^{p}(r)$ with a smaller cut-off $r_{c}$ instead of $w^{f}(r)$ with a larger cut-off $R_{c}$ for computational efficiency. The values of the other system parameters have been summarized in table 3.1 .

The physical properties of the fluid, i.e. the density and viscosity, have been chosen to be consistent with those of water. The value of $P_{0}$ has been chosen high enough to ensure small density fluctuations and a large enough velocity of sound $c_{s}$ in the 
simulation, which can be calculated as:

$$
c_{s}=\sqrt{\frac{\partial P}{\partial \rho}}=\sqrt{\frac{7 P_{0}}{\rho}}
$$

For the value of $P_{0}$ that we have used, the velocity of sound in the simulation calculated using the above equation is about $0.03 \mathrm{~m} / \mathrm{s}$. We have ensured that the velocities that we encounter in the simulations that we have performed in this study are much smaller than this velocity of sound in our simulation.

The length scale $\sigma$ has been purposefully chosen to be large for computational reasons so as to be able to use a large time-step as the main intention here is to show the two-way coupling between the polymers and the fluid through our interaction term. Although for the fluid blobs this length scale is reasonable as it is motivated phenomenologically using SPH, for the polymer blobs which are motivated using a polymer model at the mesoscopic level, it is much larger than the usual molecular level. However, if one were to use polymers which are much smaller, then one must also use smaller fluid blobs because otherwise the velocity gradients of the fluid will not be accurately experienced by the polymer blobs thereby adversely affecting the hydrodynamic coupling. This would lead to a smaller time-step and on the other hand, if larger fluid blobs are used, then the number of polymers required becomes very large and we run into computational problems again as will be described in more detail in section 3.5. Furthermore, due to the large size of the polymer blobs, the concentration $\mathrm{C}^{*}$, which is calculated as the concentration at which there is on average 1 particle in a volume of a sphere of radius $\sigma$, is rather small because this sphere is quite large. Hence, we have chosen large values for parameters like the effective Kuhn segments $p$ of our polymer blob, the strength of inter-polymer interactions $\alpha$ and the maximum number density of polymers $n_{\max }^{p}$, which is a Flory-Huggins parameter that typically corresponds to the melt density. For the Flory-Huggins interaction parameter $\chi$, we have chosen the limiting value of 0.5 , above which phase separation would occur, which is not what we aim for in this study. Nevertheless, depending on the system being modeled, i.e. depending on whether the solvent is a good solvent or a bad solvent for the polymer, the value of the Flory-Huggins parameter $\chi$ may 
be selected accordingly and our method of coupling the polymer and the fluid would still work. We want to emphasize that the term polymer blob in this chapter refers to a whole polymer molecule or even a collection of a few polymer molecules in a single blob, which is slightly different from the usual usage of the word blob referring to a collection of a few monomers.

\subsection{Results and Discussion}

\subsubsection{Pure fluid simulations}

In this subsection, we present simulations of the pure fluid, i.e. in the absence of the polymers. Since the equation of motion for the fluid is based on the Navier-Stokes equation for an incompressible Newtonian fluid, we expect to see the Newtonian behavior in the dynamic properties of the pure fluid.

\section{Equilibrium simulation}

Consider a fluid blob in a sea of fluid blobs of an incompressible Newtonian fluid in a quiescent state. At equilibrium, although the mean velocity of the fluid blob is zero, at any given instant it has a finite instantaneous velocity due to thermal fluctuations. Although these instantaneous velocities are random both in magnitude and direction, we can still say something about the distribution of these velocities and the rate at which these velocities dissipate from the kinetic and hydrodynamic theories respectively. It is well known that at thermal equilibrium, the velocities must obey the Maxwell-Boltzmann distribution and therefore for any given component of velocity $v_{0}$, we have:

$$
\left\langle v_{0}^{2}\right\rangle=k_{B} T / m
$$

where $k_{B}$ is the Boltzmann constant, $T$ is the temperature of the system and $m$ is the mass of the fluid blob. Furthermore, from hydrodynamic theory for Newtonian liquids, it is known that at short times and large wavelengths, the transverse current correlation function decays exponentially over time and the time constant is related 
to the kinematic shear viscosity of the system. So, we have calculated the transverse current correlation function $C_{t}(k, t)$ for velocities in the y-direction and wave vectors in the $\mathrm{x}$-direction $\left(\mathbf{k}_{x}=k \hat{\mathbf{e}}_{x}\right)$ as shown below:

$$
C_{t}(k, t)=\frac{1}{N_{f}}\left\langle\tilde{j}_{y}\left(\mathbf{k}_{x}, t\right) \tilde{j}_{y}^{*}\left(\mathbf{k}_{x}, 0\right)\right\rangle,
$$

where $N_{f}$ is the total number of fluid blobs, and the transverse current $\tilde{j}_{y}\left(\mathbf{k}_{\mathbf{x}}, t\right)$ is defined as follows:

$$
\tilde{j}_{y}\left(\mathbf{k}_{\mathbf{x}}, t\right)=\hat{\mathbf{e}}_{y} \cdot \sum_{j=1}^{N_{f}} \mathbf{v}_{j}(t) e^{-i \mathbf{k}_{x} \cdot \mathbf{r}_{j}(t)},
$$

where $\mathbf{r}_{j}(t)$ is the position of fluid blob $j$ at time $t$ and $\mathbf{v}_{j}(t)$ is its instantaneous velocity at that time. From hydrodynamic theory, it is known that at short times and small $k$-values, the transverse current correlation function $C_{t}(k, t)$, defined above, decays as follows[49]:

$$
C_{t}(k, t)=\left\langle v_{0}^{2}\right\rangle e^{-v k^{2} t}
$$

where $v$ is the kinematic shear viscosity of the fluid and $v_{0}$ is the initial value of the y-component of the velocity. Combining eq.(3.31) and eq.(3.34), we have:

$$
C_{t}(k, t)=\left(\frac{k_{B} T}{m}\right) e^{-v k^{2} t} .
$$

Since we need small $k$-values, i.e. large wavelengths in the x-direction, we have chosen a simulation box with a length $L_{x}=33.75 \sigma$ in the x-direction and $10 \sigma$ in the other two-directions and three different $\mathrm{k}$ values of $2 \pi / L_{x}, 4 \pi / L_{x}$ and $6 \pi / L_{x}$, i.e. $0.1862 \sigma^{-1}, 0.3723 \sigma^{-1}$ and $0.5585 \sigma^{-1}$ respectively. As we can see from Fig.3.1, there is a good match between the simulation results and the theoretical curves, particularly for the smallest $k$-value at small times, as was to be expected, indicating that the model is hydrodynamically consistent. Furthermore, it ascertains that the velocity fluctuation terms that we have derived based on the Fokker Planck equation in the appendix 3.6.2 do indeed maintain the correct kinetic temperature and that the system has the right kinematic shear viscosity. 


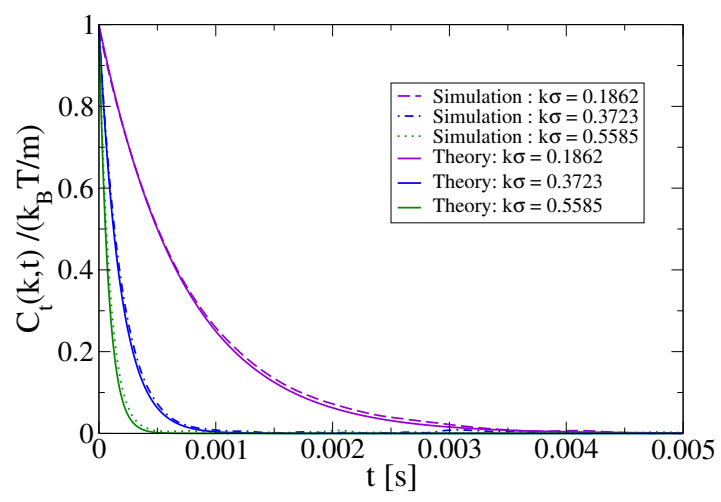

Figure 3.1: Normalized transverse current correlation function for the pure fluid at equilibrium for three different wave vectors.

\section{Shear flow simulations}

We have performed shear flow simulations with our fluid model for a number of different shear rates. Essentially, in every simulation, we have maintained a constant shear rate across the box using the Lees-Edwards technique[50] and measured the average stress in the box over a period of time. From this, we have calculated how the viscosity varies with the shear rate as shown in Fig.3.2.

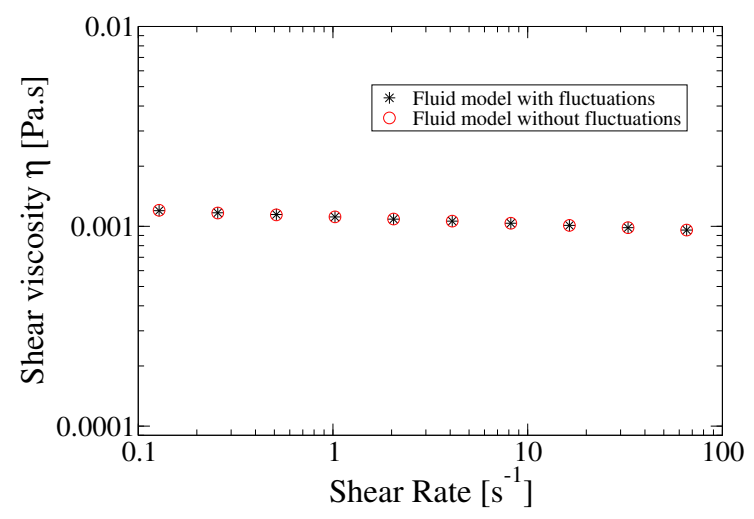

Figure 3.2: Flow curve for the pure fluid model with and without thermal fluctuations

We have performed these shear flow simulations using our fluid model with ther- 
mal fluctuations as well as by turning off the fluctuations. As we can see from Fig.3.2, the viscosity is close to the viscosity of water ( $1 \mathrm{mPa} . \mathrm{s})$ for the range of shear rates that we have considered, given the parameters and resolution of the fluid that we have used. Although the viscosity does vary by about $15 \%$, this is to be expected given the finite resolution of the fluid typically used in SPH simulations. More importantly, it is consistent with the viscosity that can be interpreted from the transverse current correlation function shown in the previous subsection 3.4.1.

\section{Reverse Poiseuille flow simulation}

In this subsection, we present simulations where the shear rate varies across the box in the y-direction as opposed to the simulations shown in the previous subsection 3.4.1, where there was a homogeneous shear flow. We essentially achieve this by applying a constant gravitational force field in the x-direction in the top half of the box and an equal and opposite field in the lower half of the box which leads to the development of a Poiseuille flow profile in both halves of the box. This method is what is commonly referred to as 'Reverse Poiseuille flow' in the literature.[51] We have performed this simulation for the fluid model with and without fluctuations and compared the results with the analytical profile that can be calculated from theory based on the NavierStokes equation for a Newtonian fluid. As can be seen from Fig.3.3, there is a good match between simulations and theory.

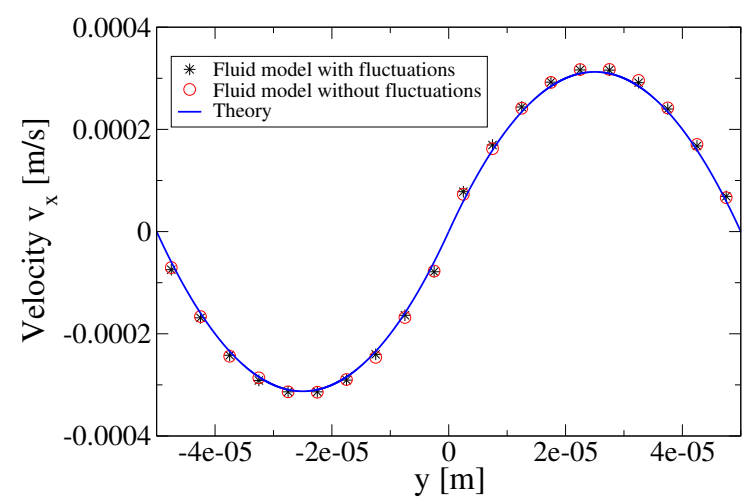

Figure 3.3: Reverse Poiseuille flow profile - Comparison of simulation results with theory 
Furthermore, it can be seen from Fig.3.2 and Fig.3.3 that the fluctuation terms don't affect the fluid viscosity. Hence, for the sake of computational efficiency, we do not include the thermal fluctuations in section 3.4.2 where we have polymer blobs as well.

\subsubsection{Polymer solution simulations}

For this study, we have considered an aqueous solution of a model polymer system, where the polymer blobs interact with each other through the conservative and transient interactions formulated by the RaPiD model mentioned in the subsections 3.3.1 and 3.3.2 of the previous section. The model for the aqueous solvent is the same as the one we have presented in the previous subsection 3.4.1 but without thermal fluctuations. The polymers and the fluid blobs of the aqueous solvent interact with each other through the interaction terms that we have defined in section 3.2.

\section{Shear flow simulations}

We have performed shear flow simulations for our model linear polymer solutions of two different concentrations $C_{1}=2.5 \mathrm{C}^{*}$ and $C_{2}=5 \mathrm{C}^{*}$ and the results have been plotted in Fig.3.4. The different contributions to the shear viscosity due to the polymers, the fluid and the interaction term have been shown. We have fitted the total shear viscosity flow curve with the Carreau Model, which is commonly used for polymer solutions, given by[52]:

$$
\frac{\eta-\eta_{\infty}}{\eta_{0}-\eta_{\infty}}=\left[1+(\lambda \dot{\gamma})^{2}\right]^{\frac{n-1}{2}}
$$

where $\eta_{0}$ is the zero shear rate viscosity, $\eta_{\infty}$ is the infinite shear rate viscosity, $\lambda$ is a parameter with units of time and $n$ is a dimensionless parameter. 
Table 3.2: Parameters for the Carreau model fit

\begin{tabular}{lllll}
\hline \hline Fit parameter & Symbol & $C_{1}$ & $C_{2}$ & Unit \\
\hline Zero shear rate viscosity & $\eta_{0}$ & 2.5260 & 6.6688 & $\mathrm{mPa} . \mathrm{s}$ \\
Infinite shear rate viscosity & $\eta_{\infty}$ & 0.9 & 0.9 & $\mathrm{mPa} . \mathrm{s}$ \\
Fit parameter 1 & $\lambda$ & 3.0504 & 2.1408 & $\mathrm{~s}$ \\
Fit parameter 2 & $n$ & 0.3441 & 0.0008 & - \\
\hline \hline
\end{tabular}

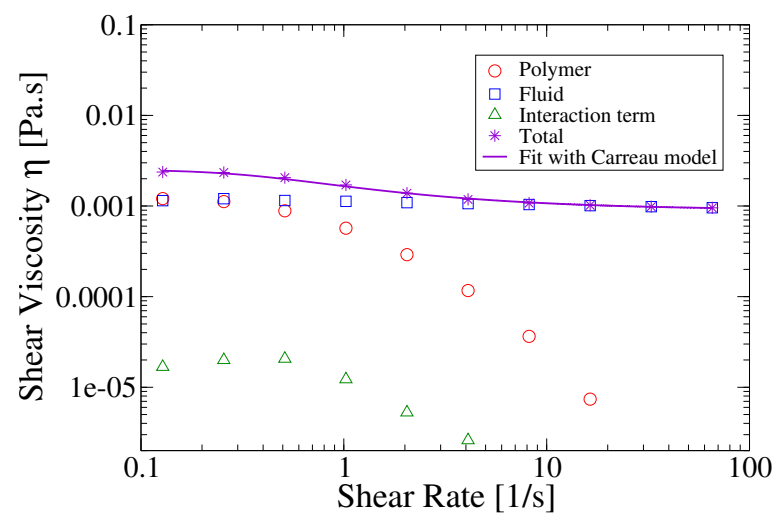

(a) Concentration $C_{1}=2.5 \mathrm{C}^{*}$

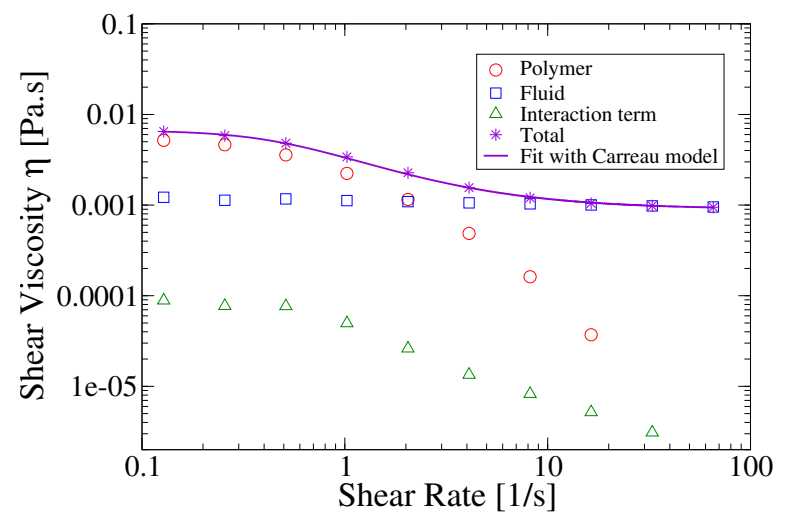

(b) Concentration $C_{2}=5 \mathrm{C}^{*}$

Figure 3.4: Flow curves for different concentrations of polymer solution showing split-up of the various contributions to the shear viscosity 
The fit parameters of the Carreau model, that best represents the total viscosity for the two different concentrations of polymer solutions that we have studied are presented in table 3.2. As can be seen from Fig.3.4, the solution is non-Newtonian in nature and the effect is clearly significant at higher concentrations. This shear thinning is mainly due to the transient interactions between the polymer blobs which are weaker at higher shear rates.

\section{Reverse Poiseuille flow simulations}

We have also performed Reverse Poiseuille flow simulations for our model linear polymer solutions at the two concentrations $C_{1}=2.5 \mathrm{C}^{*}$ and $C_{2}=5 \mathrm{C}^{*}$. We have compared the results from our simulations with CFD simulations performed with the commercial package COMSOL,[53] to which we fed as input the Carreau model flow curve that we have shown in the previous subsection 3.4.2. The results have been plotted in Fig.3.5.

As can be seen from Fig.3.5, there is no lag between the polymer blobs and the fluid blobs as their velocity profiles coincide with each other, which indicates that our interaction term couples the fluid and the polymers well. Furthermore, our model for the non-Newtonian polymer solutions is able to reproduce the characteristic flattening of the velocity profile, as is evident by comparison with the parabolic Newtonian profile particularly for higher concentrations.

Our simulations also show the cross-stream migration phenomenon observed for polymers in solution.[54] As can be seen from Fig.3.6, the concentration of water blobs remains constant along the y-axis, i.e. along the gradient direction. The polymer concentration, however, has minima at the regions of maximum shear and maxima at the regions of zero shear. Notice that the densities of the blobs have been normalized with their respective mean density in that particular solution. Therefore, the maximum concentration of the polymers in the zero-shear region in absolute terms is higher for the polymer solution with concentration $C_{2}$ as compared to that for the polymer solution with concentration $C_{1}$, although it might seem otherwise on a first glance by looking at the normalized densities plotted in Fig.3.6 


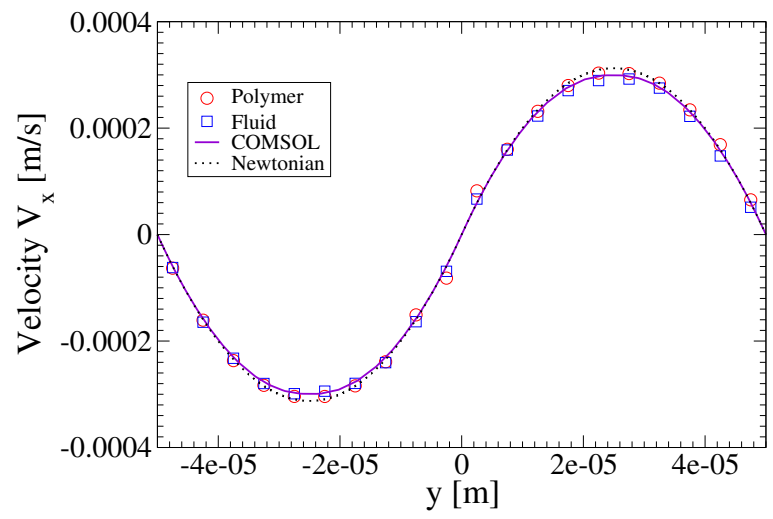

(a) Concentration $C_{1}=2.5 \mathrm{C}^{*}$

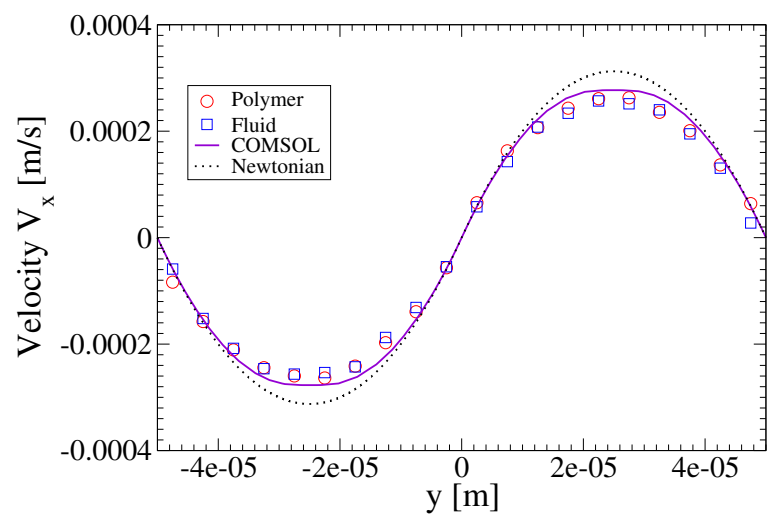

(b) Concentration $C_{2}=5 \mathrm{C}^{*}$

Figure 3.5: Reverse Poisenille Flow profiles for different concentrations of polymer solutions showing the comparison of the polymer and fluid velocity profiles in the simulation with the CFD profile generated using COMSOL and the analytical Newtonian profile 


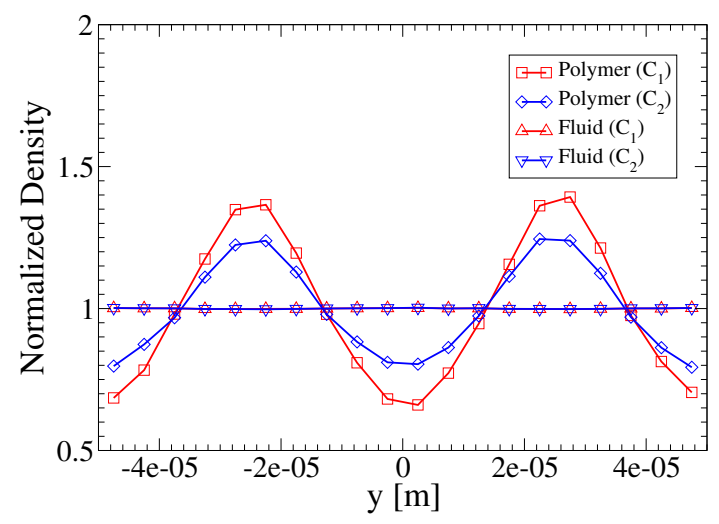

Figure 3.6: Normalized Density profile for fluid and polymer blobs for different concentrations of polymer solutions viz. $C_{1}=2.5 C^{*}$ and $C_{2}=5 C^{*}$. Note that the density of the blobs has been normalized with mean density of those blobs calculated with the respective weight function in that particular simulation.

\subsection{Conclusions}

We have presented a novel technique, hereafter referred to as Hydrodynamically Coupled Brownian Dynamics (HCBD), which is essentially a momentum conserving twoway coupling algorithm that couples the motion of polymers moving as per Brownian Dynamics with the motion of the fluid which is calculated using the SPH methodology based on the numerical solution of the Navier-Stokes equation discretized on a moving grid of fluid particles. We have calculated fluctuation terms for the update of the fluid velocities based on the Fokker Planck equation, which effectively renders our fluid model into a discretized version of fluctuating hydrodynamics.[42, 43, 44, 45] We essentially arrive at the result for an incompressible SDPD fluid derived using the GENERIC approach.[23, 24] Nevertheless, for computational efficiency, these fluctuating terms may be dropped out and then the pure fluid model, i.e. in the absence of the polymers reduces to the standard SPH formulation. The main achievement of this study is the construction of the interaction term between the Brownian polymer model and the SPH fluid model. This interaction term couples the motion of the polymers to that of the fluid without any lag and also conserves momentum at a local 
level, preserving long range hydrodynamics.

The merits of this HCBD model as a technique for modeling self-developing flow of polymer solutions can be better understood if it is compared with a standard Brownian dynamics simulation. In a standard Brownian dynamics simulation, if the friction is to be applied with respect to a non-static background fluid, then an additional term describing the motion of the background fluid must be added to the Brownian dynamics propagator. However, typically the background fluid velocity is not known a priori particularly if the fluid is flowing through a complex geometry. Even if independent simulations are carried out to determine the flow profile of the fluid, which then is added to the motion of the Brownian propagator for the polymers, there is still a lag observed between the polymers and the fluid. More importantly, the fluid velocity profile experiences no influence from the motion of the polymers, thereby making it a one-way coupling scheme. In our HCBD scheme on the other hand, the fluid and the polymer are coupled with each other through the frictional interaction term which does not lead to any lag and makes it a two-way coupling scheme. This enables us to model the flow of the polymer solution as it develops in space and time. Furthermore, since our fluid model is based on SPH, it is easy to incorporate boundary conditions such as the no-slip boundary condition at the solid interfaces in a similar way to what we have shown in an earlier work.[39]

One of the consequences of modeling the polymers at a mesoscopic scale while describing the fluid using a phenomenological approach is that if one wishes to accurately model the scale of the polymers, then one must also use fluid blobs which are not orders of magnitude larger, otherwise velocity gradients of the fluid are no longer captured accurately. This can affect the hydrodynamics of the system adversely. Even so, if larger fluid blobs are used so that a larger time-step may be used, still the ratio between the number of polymers and the number of fluid blobs becomes large, making the simulation computationally intensive. However, the computation time may be substantially reduced by massive parallelization, which in principle, our propagators allow for and it would still be faster than an MD simulation of course as we exploit the advantage of coarse-graining. 
It is also interesting to note that if one imagines a hypothetical situation in which a fluid blob is centered at the position of the center-of-mass of each polymer blob, not necessarily the same fluid blob at all times, then the position update for the polymer blob and the velocity update for the fluid blob more-or-less reduce to that of the model described in our recently published work, in which the fluid velocities are calculated at the positions of the centres-of-mass of the polymers and hence no interpolation is required.[38, 39] In that model, it was proposed that the force on the polymers be immediately transmitted to the fluid, the rationality of which, in a way, has been justified through this chapter, where we indeed arrive at it through the construction of the frictional force between polymers and the fluid which couples the two together. Having said that, the model presented in this present chapter has several advantages over the earlier model such as being able to independently choose an equation of state for the fluid through the pressure term and being able to independently decide the resolution of fluid irrespective of the concentration of the polymers. Moreover, although it might seem that the earlier model is more efficient than the present model because the former model did not have to move the fluid around separately, yet the latter model can actually turn out to be computationally more efficient in certain cases. This is because we can reduce the number of fluid particles in the system by choosing a resolution independent of the concentration of the polymers limited only by the dimensions of the complex geometry through which it flows.

Another important point is that the methodology presented in this chapter allows one to use any polymer model of one's choice and combine it with the SPH fluid model. The polymer model that we have used in this study, i.e. RaPiD has the limitation that it does not effectively capture all the internal dynamics of the polymer molecules but rather mainly relies on inter-polymer interactions to produce a nonNewtonian response like the shear-thinning behavior. However, as mentioned before, using our technique of coupling presented in this chapter i.e. HCBD, one can couple the SPH based fluid model to any other polymer model. For instance, one could use an extension of the RaPiD model,[37] where the polymer is not just represented by its center-of-mass alone but by a finitely extensible non-linear elastic (FENE) dumb- 
bell that interacts with other such dumbbells using the usual RaPiD potentials. This would be one way of incorporating some internal dynamics into the polymer model thereby more accurately modeling viscoelastic polymeric liquids without affecting the computational efficiency too much. This provides scope for further research on this topic.

\subsection{Appendix}

\subsubsection{Proof of local momentum conservation for the interaction between poly- mer and fluid blobs}

Using eq.(3.4) and eq.(3.14), we obtain the total force due to polymer blob $a$ on the fluid in its local vicinity, denoted by $\mathbf{F}_{a}^{f p}$, as follows:

$$
\mathbf{F}_{a}^{f p}=\xi \sum_{i=1}^{N_{f}} \frac{w^{f}\left(r_{a i}\right)}{n_{i}^{f}}\left(\frac{\mathrm{d} \mathbf{r}_{a}^{*}}{\mathrm{~d} t}-\sum_{j=1}^{N_{f}} \frac{w^{f}\left(r_{a j}\right)}{n_{j}^{f}} \mathbf{v}_{j}\right)
$$

Simplifying the first term using eq.(3.5) and rewriting the second term as two terms one where $j=i$ and one where $j \neq i$, we obtain:

$$
\mathbf{F}_{a}^{f p}=\xi\left[\frac{\mathrm{d} \mathbf{r}_{a}^{*}}{\mathrm{~d} t}-\sum_{i=1}^{N_{f}}\left(\frac{w^{f}\left(r_{a i}\right)}{n_{i}^{f}}\right)^{2} \mathbf{v}_{i}-\left(\sum_{i=1}^{N_{f}} \frac{w^{f}\left(r_{a i}\right)}{n_{i}^{f}} \sum_{j \neq i}^{N_{f}} \frac{w^{f}\left(r_{a j}\right)}{n_{j}^{f}} \mathbf{v}_{j}\right)\right]
$$

Rewriting the last term and simplifying, we get:

$$
\mathbf{F}_{a}^{f p}=\xi\left[\frac{\mathrm{d} \mathbf{r}_{a}^{*}}{\mathrm{~d} t}-\sum_{i=1}^{N_{f}}\left(\frac{w^{f}\left(r_{a i}\right)}{n_{i}^{f}}\right)^{2} \mathbf{v}_{i}-\left(\sum_{j=1}^{N_{f}} \frac{w^{f}\left(r_{a j}\right)}{n_{j}^{f}} \mathbf{v}_{j} \sum_{i \neq j}^{N_{f}} \frac{w^{f}\left(r_{a i}\right)}{n_{i}^{f}}\right)\right]
$$

Using eq.(3.5) and rewriting the last term, we obtain:

$$
\mathbf{F}_{a}^{f p}=\xi\left[\frac{\mathrm{d} \mathbf{r}_{a}^{*}}{\mathrm{~d} t}-\sum_{i=1}^{N_{f}}\left(\frac{w^{f}\left(r_{a i}\right)}{n_{i}^{f}}\right)^{2} \mathbf{v}_{i}-\sum_{j=1}^{N_{f}} \frac{w^{f}\left(r_{a j}\right)}{n_{j}^{f}} \mathbf{v}_{j}\left(1-\frac{w^{f}\left(r_{a j}\right)}{n_{j}^{f}}\right)\right]
$$

Simplifying further and noting that two of the terms cancel with each other, and replacing $j$ with $i$, we get:

$$
\mathbf{F}_{a}^{f p}=\xi\left(\frac{\mathrm{d} \mathbf{r}_{a}^{*}}{\mathrm{~d} t}-\sum_{i=1}^{N_{f}} \frac{w^{f}\left(r_{a i}\right)}{n_{i}^{f}} \mathbf{v}_{i}\right)
$$


which is clearly equal and opposite to the total force exerted by the fluid on the polymer blob a, i.e. $\mathbf{F}_{a}^{p f}$ shown in eq.(3.7).

\subsubsection{Derivation of stochastic update for the fluid velocities}

In this appendix, we derive the statistical properties of the velocity fluctuations $\mathrm{d} \mathbf{W}_{j k}^{v}$ for the pure fluid at quiescent state. Although we use a different approach, we essentially arrive at the same result as the velocity fluctuations for an incompressible SDPD fluid derived using the GENERIC approach.[23, 24]

According to the Chapman-Kolmogorov equation, the probability to find the system at time $t+\mathrm{d} t$ at the point $\mathbf{z}$ in the phase space characterised by positions and velocities i.e. $\mathbf{z}=\left\{\mathbf{r}_{1}, \ldots, \mathbf{r}_{N}, \mathbf{v}_{1}, \ldots, \mathbf{v}_{N}\right\}$, given that at $t=0$ the system was at point $\mathbf{z}_{0}$ in the phase space is given by:

$$
\mathscr{G}\left(\mathbf{z} ; \mathbf{z}_{0} ; t+\mathrm{d} t\right)=\int \mathrm{d}^{6 N} z^{\prime} \mathscr{G}\left(\mathbf{z} ; \mathbf{z}^{\prime} ; \mathrm{d} t\right) \mathscr{G}\left(\mathbf{z}^{\prime} ; \mathbf{z}_{0} ; t\right),
$$

i.e. where $\mathbf{z}^{\prime}$ is any intermediate point in the phase space, through which the system could have passed at time $t$. As $\lim _{\mathrm{d} t \rightarrow 0} \mathscr{G}\left(\mathbf{z} ; \mathbf{z}^{\prime} ; \mathrm{d} t\right)=\delta\left(\mathbf{z}-\mathbf{z}^{\prime}\right)$. Going through the usual derivation, we arrive at the following Fokker-Planck equation:

$$
\begin{aligned}
& \frac{\partial \mathscr{G}\left(\mathbf{z} ; \mathbf{z}_{0} ; t\right)}{\partial t} \\
& =-\sum_{i} \sum_{\alpha} \frac{\partial}{\partial r_{i, \alpha}}\left\{\left(v_{i, \alpha}\right) \mathscr{G}\left(\mathbf{z} ; \mathbf{z}_{0} ; t\right)\right\} \\
& \quad-\sum_{i} \sum_{\alpha} \frac{\partial}{\partial v_{i, \alpha}}\left\{\left(\sum_{j} \frac{f_{i j}}{m}\left(v_{j, \alpha}-v_{i, \alpha}\right)+\frac{F_{i, \alpha}}{m}\right) \mathscr{G}\left(\mathbf{z} ; \mathbf{z}_{0} ; t\right)\right. \\
& \left.\quad-\frac{1}{2} \sum_{j} \sum_{k} \sum_{l} \sum_{\beta} \frac{\partial}{\partial v_{i, \beta}}\left[\frac{\left\langle\mathrm{d} W_{i k, \alpha}^{v} \mathrm{~d} W_{j l, \beta}^{v}\right\rangle}{\mathrm{d} t} \mathscr{G}\left(\mathbf{z} ; \mathbf{z}_{0} ; t\right)\right]\right\},
\end{aligned}
$$

where $\alpha$ and $\beta$ run from 1 to 3 , corresponding to the different Cartesian components and $F_{i}$ represents the force due to the pressure gradient term as shown below:

$$
\frac{F_{i}}{m}=\frac{(\nabla P)_{i}}{\rho_{i}}=\nabla\left(\frac{P}{\rho}\right)_{i}+\frac{P_{i}}{\rho_{i}^{2}}(\nabla \rho)_{i} .
$$


Since the standard SPH formalism for a gradient of any property A is given by $(\nabla A)_{i}=\sum_{j=1}^{N} \frac{m_{i} A_{i}}{\rho_{i}} \frac{\mathrm{d} w}{\mathrm{~d} r}\left(r_{i j}\right) \frac{\mathbf{r}_{i j}}{r_{i j}}$, we get:

$$
F_{i}=\sum_{j=1}^{N_{f}}\left(\frac{P_{j}}{\left(n_{j}^{f}\right)^{2}}+\frac{P_{i}}{\left(n_{i}^{f}\right)^{2}}\right) \frac{\mathrm{d} w^{f}}{\mathrm{~d} r}\left(r_{i j}\right) \frac{\mathbf{r}_{i j}}{r_{i j}}
$$

which is the form that has been shown in eq.(3.11) and used thereafter in eq.(3.16).

At steady state, we expect the left hand side of the eq.(3.43) to be zero. Furthermore, we expect that for a system in a quiescent state, the equilibrium distribution of $\mathbf{z}$ is given by the Maxwell-Boltzmann distribution:

$$
\mathscr{G}^{e q}(\mathbf{z}) \propto \exp \left\{\frac{1}{k_{B} T} \Phi\left(\mathbf{r}_{1}, \ldots, \mathbf{r}_{N}\right)-\frac{1}{2 k_{B} T} \sum_{i} m \mathbf{v}_{i} \cdot \mathbf{v}_{i}\right\},
$$

where $\Phi$ is the total potential energy based on the pressure distribution of the system given by $\Phi=P V=\sum_{i=1}^{N_{f}} P_{i} V / N_{f}$, where $N_{f} / V$ may be replaced by $\bar{n}^{f}$, from which the conservative forces can be calculated as $\mathbf{F}_{i}=-\partial \Phi / \partial \mathbf{r}_{i}$. At equilibrium, $\mathscr{G}\left(\mathbf{z} ; \mathbf{z}_{0} ; t\right)$ in Eq. (3.43) must be replaced by $\mathscr{G}^{e q}(\mathbf{z})$.

We pre-calculate some of the derivatives that will be required as follows:

$$
\begin{aligned}
\frac{\partial}{\partial \mathbf{r}_{i}} \mathscr{G}^{e q}(\mathbf{z}) & =-\frac{1}{k_{B} T}\left(\frac{\partial}{\partial \mathbf{r}_{i}} \Phi\right) \mathscr{G}^{e q}(\mathbf{z})=\frac{\mathbf{F}_{i} \mathscr{G}^{e q}(\mathbf{z})}{k_{B} T} \\
\frac{\partial}{\partial \mathbf{v}_{i}} \mathscr{G}^{e q}(\mathbf{z}) & =-\frac{m \mathbf{v}_{i} \mathscr{G}^{e q}(\mathbf{z})}{k_{B} T} .
\end{aligned}
$$

Notice that these imply

$$
\sum_{i} \sum_{\alpha} \frac{\partial}{\partial r_{i, \alpha}} v_{i, \alpha} \mathscr{G}^{e q}(\mathbf{z})+\sum_{i} \sum_{\alpha} \frac{\partial}{\partial v_{i, \alpha}} \frac{F_{i, \alpha}}{m} \mathscr{G}^{e q}(\mathbf{z})=0 .
$$

Therefore, these terms will get eliminated from Eq. (3.43) at equilibrium. As a result, all remaining sums between curly brackets in Eq. (3.43) with $\mathscr{G}\left(\mathbf{z} ; \mathbf{z}_{0} ; t\right)$ replaced by $\mathscr{G}^{e q}(\mathbf{z})$ must be identically equal to zero.

We re-write the correlations of the velocity fluctuations as follows:

$$
\left\langle\mathrm{d} W_{i k, \alpha}^{v} \mathrm{~d} W_{j l, \beta}^{v}\right\rangle=2 k_{B} T C_{i k \alpha j l \beta}^{v v} \mathrm{~d} t
$$


Thus, at equilibrium, we must have:

$$
\sum_{j} \frac{f_{i j}}{m}\left(v_{j, \alpha}-v_{i, \alpha}\right)+\sum_{j} \sum_{k} \sum_{l} \sum_{\beta} C_{i k \alpha j l \beta}^{v v} m v_{i, \beta}=0
$$

These equations will be identically satisfied if we choose:

$C_{i k \alpha j l \beta}^{v v}=\delta_{\alpha \beta}\left[\left(f_{i k} / m^{2}\right) \delta_{i j} \delta_{k l}-\left(f_{k i} / m^{2}\right) \delta_{i l} \delta_{j k}\right]$.

Thus, in conclusion, to get the equilibrium distribution at steady state, we must choose the stochastic variables according to:

$$
\begin{aligned}
\left\langle\mathrm{d} \mathbf{W}_{i j}^{v} \mathrm{~d} \mathbf{W}_{i j}^{v}\right\rangle & =\left(\frac{2 k_{B} T}{m}\right)\left(\frac{\mathrm{d} t}{m}\right) f_{i j} \mathbf{I} \\
\left\langle\mathrm{d} \mathbf{W}_{i k}^{v} \mathrm{~d} \mathbf{W}_{j l}^{v}\right\rangle & =\mathbf{0} \quad(i k \neq j l \wedge i k \neq l j),
\end{aligned}
$$




\section{Bibliography}

[1] B. Dünweg and K. Kremer, Physical Review Letters 66, 2996 (1991).

[2] B. Dünweg and K. Kremer, The Journal of Chemical Physics 99, 6983 (1993).

[3] P. Ahlrichs and B. Dünweg, The Journal of Chemical Physics 111, 8225 (1999).

[4] A. Malevanets and R. Kapral, The Journal of Chemical Physics 110, 8605 (1999).

[5] A. Malevanets and R. Kapral, The Journal of Chemical Physics 112, 7260 (2000).

[6] A. Malevanets and J. Yeomans, Europhysics Letters 52, 231 (2000).

[7] M. Ripoll, K. Mussawisade, R. Winkler, and G. Gompper, Europhysics Letters 68, 106 (2004).

[8] R. Kapral, Advances in Chemical Physics 140, 89 (2008).

[9] G. Gompper, T. Ihle, D. Kroll, and R. Winkler, Advances in Polymer Science 221, 1 (2009).

[10] C.-C. Huang, R. G. Winkler, G. Sutmann, and G. Gompper, Macromolecules 43, 10107 (2010).

[11] P. J. Hoogerbrugge and J. M. V. A. Koelman, Europhysics Letters 19, 155 (1992).

[12] J. M. V. A. Koelman and P. J. Hoogerbrugge, Europhysics Letters 21, 363 (1993).

[13] P. Español and P. Warren, Europhysics Letters 30, 191 (1995).

[14] Y. Kong, C. Manke, W. Madden, and A. Schlijper, International Journal of Thermophysics 15, 1093 (1994). 
[15] A. Schlijper, P. Hoogerbrugge, and C. Manke, Journal of Rheology 39, 567 (1995).

[16] X. Fan, N. Phan-Thien, N. T. Yong, X. Wu, and D. Xu, Physics of Fluids 15, 11 (2003).

[17] X. Fan, N. Phan-Thien, S. Chen, X. Wu, and T. Yong Ng, Physics of Fluids 18, 063102 (2006).

[18] J. P. Hernández-Ortiz, H. Ma, J. J. de Pablo, and M. D. Graham, Physics of Fluids 18, 123101 (2006).

[19] J. A. Millan, W. Jiang, M. Laradji, and Y. Wang, The Journal of Chemical Physics 126, 124905 (2007).

[20] D. A. Fedosov, G. Em Karniadakis, and B. Caswell, The Journal of Chemical Physics 128, 144903 (2008).

[21] J. A. Millan and M. Laradji, Macromolecules 42, 803 (2009).

[22] Z. Li, Y. Li, Y. Wang, Z. Sun, and L. An, Macromolecules 43, 5896 (2010).

[23] P. Español and M. Revenga, Physical Review E 67, 026705 (2003).

[24] A. Vázquez-Quesada, M. Ellero, and P. Español, The Journal of Chemical Physics 130, 034901 (2009).

[25] L. B. Lucy, The Astronomical Journal 82, 1013 (1977).

[26] R. A. Gingold and J. J. Monaghan, Monthly Notices of the Royal Astronomical Society 181, 375 (1977).

[27] R. Gingold and J. Monaghan, Journal of Computational Physics 46, 429 (1982).

[28] J. J. Monaghan, Annual Review of Astronomy and Astrophysics 30, 543 (1992).

[29] J. J. Monaghan, Reports on Progress in Physics 68, 1703 (2005).

[30] S. Litvinov, M. Ellero, X. Hu, and N. A. Adams, Physical Review E 77, 066703 (2008). 
[31] S. Litvinov, Q. Xie, X. Hu, N. Adams, and M. Ellero, Fluids 1, 7 (2016).

[32] P. Español and P. B. Warren, The Journal of Chemical Physics 146, 150901 (2017).

[33] A. Van den Noort, W. K. den Otter, and W. J. Briels, Europhysics Letters 80, 28003 (2007).

[34] W. J. Briels, Soft Matter 5, 4401 (2009).

[35] I. S. Santos de Oliveira, A. van den Noort, J. T. Padding, W. K. den Otter, and W. J. Briels, The Journal of Chemical Physics 135, 104902 (2011).

[36] I. S. Santos de Oliveira, W. K. den Otter, and W. J. Briels, The Journal of chemical physics 137, 204908 (2012).

[37] I. S. Santos de Oliveira, B. W. Fitzgerald, W. K. den Otter, and W. J. Briels, The Journal of Chemical Physics 140, 104903 (2014).

[38] J. T. Padding and W. J. Briels, The Journal of Chemical Physics 141, 244108 (2014).

[39] V. R. Ahuja, J. van der Gucht, and W. J. Briels, The Journal of Chemical Physics 145, 194903 (2016).

[40] W. J. Briels, Theory of Polymer Dynamics. (Lecture Notes, Uppsala, Sweden, 1994).

[41] C. W. Gardiner et al., Handbook of Stochastic Methods, Vol. 4 (Springer Berlin, 1985).

[42] L. Landau and E. Lifshitz, Statistical Physics (Pergamon Press, 1958).

[43] L. Landau and E. Lifshitz, Fluid Mechanics (Pergamon Press, 1959).

[44] J. M. O. De Zárate and J. V. Sengers, Journal of Statistical Physics 115, 1341 (2004). 
[45] J. M. O. De Zárate and J. V. Sengers, Hydrodynamic Fluctuations in Fluids and Fluid Mixtures (Elsevier, 2006).

[46] B. Hoomans, J. Kuipers, W. J. Briels, and W. P. M. van Swaaij, Chemical Engineering Science 51, 99 (1996).

[47] G. K. Batchelor, An Introduction to Fluid Dynamics (Cambridge university press, 2000).

[48] R. H. Cole, Underwater Explosions (Dover Publications, 1965).

[49] J. P. Boon and S. Yip, Molecular Hydrodynamics (Courier Corporation, 1980).

[50] A. Lees and S. Edwards, Journal of Physics C: Solid State Physics 5, 1921 (1972).

[51] D. A. Fedosov, G. E. Karniadakis, and B. Caswell, The Journal of Chemical Physics 132, 144103 (2010).

[52] R. Bird, W. Stewart, and E. Lightfoot, Transport Phenomena, Wiley International edition (Wiley, 2007).

[53] Comsol, Multiphysics User Guide for COMSOL 5.0 (2014).

[54] L. Fang, H. Hu, and R. G. Larson, Journal of Rheology 49, 127 (2005). 



\section{Shear Banding and Shear Rolls}

We present coarse-grain particle-based simulations for studying rheology of complex soft matter systems such as solutions of large polymers or micro-gel particles, wherein the solute and the solvent are both explicitly modeled using mesoscopic blobs. We use our recently published two-way coupling technique called Hydrodynamically Coupled Brownian Dynamics (HCBD) for coupling large mesoscopic particles to the solvent [J. Chem. Phys. 148, 034902 (2018)]. However, for this study, we use a slightly less coarse-grained and hence more accurate polymer model to model our polymers or micro-gel particles. We employ Finitely Extensible Nonlinear Elastic (FENE) dumbells to represent our mesoscopic particles, which thereby render them elastically deformable up to a finite length. Each lobe of the dumbell interacts with other such lobes in solution based on the pair-potentials of the Responsive Particle Dynamics (RaPiD) model. In addition, they also experience an effective frictional force proportional to their velocity relative to the solvent flow field interpolated at their position. The motion of the solvent is calculated by solving a discretized version of the Navier-Stokes equation using the centers-of-mass of the solvent blobs as a moving grid as in a Smoothed Particle Hydrodynamics (SPH) simulation. The Navier-Stokes equation is augmented with the interaction term of the HCBD coupling technique to couple the background fluid flow field to the motion of the mesoscopic particles. Using this model, we study the rheology of our model system under shear using the Lees-Edwards method. We observe shear banding and the spontaneous formation of interesting microstructures due to elastic instabilities occurring in bulk shear flow. 


\subsection{Introduction}

It is well known that flow of complex soft matter, even through very simple geometries, does not always end up with a simple homogeneous velocity field. Among the best known inhomogeneous shear flows are the vorticity and gradient banded shear flows. In gradient banded flows the material (ideally) flows between two parallel plates, of which the upper plate moves with respect to the lower plate along a direction parallel to their common orientation. The system splits into two bands of flow parallel to the plates and with different shear rates, connected through a narrow transition region where the shear rate continuously changes from the shear rate in one band to that in the other band. The earliest and most extensively studied systems that exhibit shear banding are worm-like micellar systems. [1, 2, 3, 4] Since then shear banding has been found to occur in a great variety of systems, among which are entangled polymer solutions. $[5,6,7,8]$ Many more examples can be found in a large number of review papers that have been published by now. $[9,10,11,12,13,14,15,16]$

On the theoretical side it was quickly realized that the common description of fluid flow through a stress tensor containing local stresses had to be extended to include non-local stresses. Among the simplest ways to do this is the approach of Dhont [17], who extended the formal expansion of the stress tensor in spatial velocity gradients to include the next higher order contribution being proportional to the Laplacian of the symmetrized velocity gradient tensor. Alternatively one may add a stress-diffusion term to the equation of motion as has been advocated by Olmsted and Fielding. [12, 18] This method finds its roots in an early paper by El-Kareh and Leal. [19]

Particle based computer simulations of shear banding are relatively few because of the huge number of particles needed to simulate large amounts of solutions of complex molecules. Only very simple models allow to perform simulations of systems large enough to faithfully represent banded systems. Among these are DPD simulations of polymer solutions. [20] Since polymers in these simulations are represented as long chains of connected DPD particles they are still limited to rather small systems. An alternative is to represent polymers by single particles, dressed with as few 
additional variables as possible to mimic some of the dynamic characteristic of polymers, and to use Brownian dynamics instead of a second order propagator in order to allow for large time steps. Such a method was developed by Briels and van den Noort and goes under the name Responsive Particle Dynamics (RaPiD).[21, 22] Early examples of simulations of shear banding systems are presented in references.[23, 24, 25]

One disadvantage of standard Brownian dynamics simulations is that the particles are assumed to affinely follow some well defined background flow besides being displaced under the influence of their mutual interaction forces. In the RaPiD simulations mentioned above, an overall shear rate was imposed on the box through Lees-Edwards boundary conditions, while the local background flow was measured in slabs parallel to the flow-vorticity plane on the basis of the displacements of the particles in the slabs. By doing so, the system is allowed to develop its own flow profile instead of being forced to follow some imposed flow profile as would be the case when the SLLOD algorithm had been used [26]. This method is limited, however, to applications where the flow gradients only occur in the direction perpendicular to the slabs chosen to calculate the background flow. This means that more complicated inhomogeneities or instabilities can not be studied. Moreover, except for the simplest cases and at considerable computational costs [27], hydrodynamic interactions are not included in Brownian dynamics simulations. Both of these problems have been overcome with the Hydrodynamically Coupled Brownian Dynamics (HCBD) method that we have recently developed.[28] Furthermore, this method shares with all other particle based simulation methods the advantage that density and concentration gradients can develop simultaneously.

In this chapter, we have applied the HCBD method to study a model soft matter system in shear flow. In section 4.2, we briefly describe the HCBD model. In section 4.3, we further explicate the potentials that we have used to describe the interactions between the various particles in the system. Also in that section we present the weight functions that occur in our application of HCBD, and make some comments on the level of relative resolution of solvent and solute. We end section 4.3 by giving the parameters used to describe/define our system. Our main results and discussion are 
presented in section 4.4. We end with some conclusions and discuss scope for future research in section 4.5. Our main interest in this chapter will be to investigate if the additional flexibility of our method and the inclusion of hydrodynamic interactions give rise to instabilities and inhomogeneous flow profiles, other than those in simple shear banding.

\subsection{Model}

\subsubsection{Equation of motion for the polymer blobs}

Our interest in this chapter is in the flow properties of systems containing large mesoscopic particles, for example large polymer molecules or micro-gel particles, dissolved in appropriate solvents. In order to allow the particles to deform under stresses resulting from interactions among themselves and with the fluid, we will represent them with two mesoscopic blobs connected to each other through an elastically finitely extensible bond.

The position of the center-of-mass $\mathbf{r}_{a}(t)$ at time $t$ of any given blob $a$ will be updated as follows:

$$
\mathrm{d} \mathbf{r}_{a}=\mathbf{v}\left(\mathbf{r}_{a}\right) \mathrm{d} t+\left(\frac{\mathbf{F}_{a}}{\xi_{a}}\right) \mathrm{d} t+k_{B} T \frac{\partial}{\partial \mathbf{r}_{a}}\left(\frac{1}{\xi_{a}}\right) \mathrm{d} t+\mathrm{d} \mathbf{W}_{a}^{R}
$$

Here, $\mathbf{r}_{a}$ is shorthand for $\mathbf{r}_{a}(t)$. Similarly, $\mathbf{v}\left(\mathbf{r}_{a}\right)$ is shorthand for $\mathbf{v}\left(\mathbf{r}_{a}, t\right)$, the background fluid velocity at the position of the blob at time $t$. The latter is calculated through an interpolation based on the velocities of fluid blobs in the vicinity of blob $a$ using an appropriately normalized weight function $w^{f}(r)$ as shown below

$$
\mathbf{v}\left(\mathbf{r}_{a}\right)=\sum_{i=1}^{N_{f}} \frac{w^{f}\left(r_{a i}\right)}{n_{i}^{f}} \mathbf{v}_{i}
$$

Here $\mathrm{d} t$ is the time-increment with every step and $\mathbf{F}_{a}$ is shorthand for $\mathbf{F}_{a}(t)$, the driving force acting on blob $a$ at time $t$ resulting from interactions with other such blobs, and any force field that may have been applied. $n_{i}^{f}=\sum_{j=1}^{N_{f}} w^{f}\left(r_{i j}\right)$ is the number density of fluid blobs at the position of fluid blob $i$. 
Hereafter in this chapter, we will not include the $t$ in our notation, tacitly assuming that it is implicitly present.

We will base the calculation of forces on polymers on the RaPiD-FENE model for dumbbells, according to which the force $\mathbf{F}_{a}$ on blob $a$ contains three contributions,

$$
\mathbf{F}_{a}=-\frac{\partial}{\partial \mathbf{r}_{a}}\left[\Phi_{c}+\Phi_{t}+\Phi_{f}\right]
$$

where $\Phi_{c}$ is the so-called 'conservative' potential, $\Phi_{t}$ is the so-called 'transient' potential and $\Phi_{f}$ is the FENE potential, all of which shall be defined and described in section 4.3. $\xi_{a}$, shorthand for $\xi\left(\mathbf{r}_{a}\right)$, is the friction coefficient at the position of polymer blob $a$ and the third term on the right hand side of eq.(4.1) is a drift term accounting for the spatial variation of the friction coefficient, which we neglect in this study as we assume a constant friction coefficient for the sake of simplicity. The last term on the right hand side of eq.(4.1), i.e. $\mathrm{d} \mathbf{W}_{a}^{R}$, is a random displacement typical of Brownian dynamics simulations. This random displacement is uncorrelated in time and has a magnitude that is calculated in accordance with the fluctuation dissipation theorem, satisfying:

$$
\left\langle\mathrm{d} \mathbf{W}_{a}^{R} \mathrm{~d} \mathbf{W}_{b}^{R}\right\rangle=2 k_{B} T\left(\frac{\mathrm{d} t}{\xi_{a}}\right) \delta_{a b} \mathbf{I} .
$$

\subsubsection{Equation of motion for the fluid blobs}

The background fluid will be modeled through a collection of fluid blobs moving according to a discretization of the fluctuating Navier-Stokes equation. [29, 30] The position of the center-of-mass of fluid blob $i$ is updated according to:

$$
\mathrm{d} \mathbf{r}_{i}=\mathbf{v}_{i} \mathrm{~d} t
$$

Velocities $\mathbf{v}_{i}$ are updated according to the Smooth Particle Hydrodynamics (SPH) equations [31] to which we have added a term coupling the fluid motion to that of the polymers, as part of the HCBD two-way coupling technique. [28]. Thus, we arrive at the following equation that we have used in our simulations to update the velocity of 
any given fluid blob $i$ :

$$
\begin{aligned}
\mathrm{d} \mathbf{v}_{i}= & -\frac{\mathrm{d} t}{m}\left[\sum_{j=1}^{N_{f}}\left(\frac{P_{i}}{\left(n_{i}^{f}\right)^{2}}+\frac{P_{j}}{\left(n_{j}^{f}\right)^{2}}\right) \frac{\mathrm{d} w^{f}}{\mathrm{~d} r}\left(r_{i j}\right) \frac{\mathbf{r}_{i j}}{r_{i j}}+\sum_{j=1}^{N_{f}} f_{i j} \mathbf{v}_{i j}\right]+\mathbf{g}_{i} \mathrm{~d} t \\
& +\frac{\mathrm{d} t}{m}\left[\sum_{a=1}^{N_{b}} \frac{w^{f}\left(r_{a i}\right)}{n_{i}^{f}} \mathbf{F}_{a}\right]+\sum_{j=1}^{N_{f}} \mathrm{~d} \mathbf{W}_{i j}^{v}
\end{aligned}
$$

where $m$ is the mass of a fluid blob, $P_{i}$ is the pressure at the position of the fluid blob $i, n_{i}^{f}$ is the local number density of fluid blob $i$ as before, and $N_{b}$ is the number of polymer blobs (which is twice the number of polymers $N_{p}$ as each polymer is represented by two blobs). $\mathbf{v}_{i j}$ is the velocity of fluid blob $i$ relative to that of $j$. The sum of terms $f_{i j} \mathbf{v}_{i j}$, with $f_{i j}$ being shorthand for $f\left(r_{i j}\right)$, represents the viscous dissipation in the fluid. $f_{i j}$ is a symmetric function defined as follows:

$$
f\left(r_{i j}\right)= \begin{cases}-\left(\frac{2 \eta}{n_{i}^{f} n_{j}^{f}}\right) \frac{1}{r_{i j}} \frac{\mathrm{d} w^{f}}{\mathrm{~d} r}\left(r_{i j}\right) & \text { for } r_{i j} \leq R_{c} \\ 0 & \text { for } r_{i j} \geq R_{c}\end{cases}
$$

with $\eta$ being the solvent viscosity. $\mathbf{g}_{i}$ is the acceleration due to body forces. The pairwise velocity fluctuation terms are uncorrelated in time and have been calculated in an anti-symmetric manner such that $\mathrm{d} \mathbf{W}_{i j}^{v}=-\mathrm{d} \mathbf{W}_{j i}^{v}$ to conserve momentum. The properties of these momentum fluctuations $d \mathbf{W}_{i j}^{v}$ have been calculated in a way that the steady state probability distribution of the positions and velocities of the fluid in a quiescent state yields the expected equilibrium distribution, more details about which can be found in our recently published work.[28, 32] Thus, we have:

$$
\begin{aligned}
\left\langle\mathrm{d} \mathbf{W}_{i j}^{v} \mathrm{~d} \mathbf{W}_{i j}^{v}\right\rangle & =\left(\frac{2 k_{B} T}{m}\right)\left(\frac{\mathrm{d} t}{m}\right) f_{i j} \mathbf{I}, \\
\left\langle\mathrm{d}^{v} \mathbf{W}_{i k}^{v} \mathbf{W}_{j l}^{v}\right\rangle & =\mathbf{0} \quad(i k \neq j l \wedge i k \neq l j) .
\end{aligned}
$$

However for computational reasons, we have ignored the fluctuations of the fluid for the simulations that we have presented in this chapter. But in principle, if one would like to see the effect of fluctuations of the fluid, one may add the additional fluctuation terms mentioned above.

The remaining term in Eq. 4.6 represents the force exerted by the polymers on the 
fluid. It was first suggested by us in our recent paper where we introduced the HCBD method.[28]

\subsection{Potentials and System Parameters}

\subsubsection{Potentials}

The potential used to describe the interactions between complex polymers consists of three contributions, the FENE potential between blobs within a polymer, the conservative potential between different polymers, and the transient potential mimicking on a very coarse level memory effects resulting from the eliminated degrees of freedom.

The FENE potential $\Phi_{f}$ for the deformation of the finitely extensible nonlinear elastic polymer springs is given by:

$$
\Phi_{f}\left(r_{i}\right)=-\frac{1}{2} k r_{0}^{2} \sum_{i=1}^{N} \ln \left[1-\left(\frac{r_{i}}{r_{0}}\right)^{2}\right],
$$

where $r_{i}$ is the separation between the two lobes of the polymer dumbell $i, k$ is the spring constant, and $r_{0}$ is the maximum allowed deformation of the spring.

For simplicity the conservative potential will be expressed in terms of the centerof-mass positions of the polymers, i.e. treating the polymers as single entities. Of course, since the center-of-mass position is half the sum of the position vectors of the two blobs constituting the polymer, forces on the blobs can still be calculated as negative gradients of the potential. We use the Flory-Huggins potential free energy as a conservative potential that is rather soft, but still homogenizes the distribution of the polymers. It has been used by us on several previous occasions to simulate polymer solutions[28, 33, 34, 35], and is defined as follows:

$$
\Phi_{c}=p k_{B} T \sum_{a=1}^{N_{p}}\left[\left(\frac{1-\phi_{a}}{\phi_{a}}\right) \ln \left(1-\phi_{a}\right)-\chi \phi_{a}\right] .
$$

$\Phi_{c}$ is the conservative potential, $N_{p}$ is the total number of polymers in solution as mentioned before, $p$ is the number of Kuhn segments per polymer, $\chi$ is the solvent interaction parameter and $\phi_{a}$ is the local volume fraction of polymers in the neigh- 
borhood of polymer $a$ at time $t$ calculated as:

$$
\phi_{a}=\frac{n_{a}^{p}}{n_{\max }^{p}} .
$$

Here, $n_{\text {max }}^{p}$ is the maximum number density of polymers that the system is allowed to reach, i.e. the melt density, and $n_{a}^{p}$ is the local number density at the position of the center-of-mass of polymer $a$ calculated as:

$$
n_{a}^{p}=\sum_{b=1}^{N_{p}} w^{p}\left(r_{a b}\right)
$$

where $r_{a b}$ is the distance between the centers-of-mass of polymers $a$ and $b$ at time $t$ and $w^{p}(r)$ is a normalized weight function with a cut-off $r_{c}$. The superscript $p$ in $w^{p}(r)$ indicates that this is the weight function used for the polymer blobs.

The transient potential, taken from the RaPiD model, essentially takes into account the history of the interacting polymer blobs by keeping track of an additional set of dynamic variables $\lambda_{a b}$, and is given by:[21, 22]

$$
\Phi_{t}=\frac{1}{2} \alpha \sum_{a, b=1}^{N_{b}}\left(\lambda_{a b}-\lambda_{a b}^{e q}\right)^{2} .
$$

Here, $\Phi_{t}$ the transient potential, $\alpha$ is a parameter associated with the strength of the interactions or in other words the penalty for the deviation of the dynamic variable $\lambda_{a b}$ from its equilibrium value $\lambda_{a b}^{e q}$. The variable $\lambda_{a b}$ is introduced to represent the degree of intermixing of the polymers $a$ and $b$, which evolves over time based on the following first order stochastic differential equation:

$$
\mathrm{d} \lambda_{a b}=-\left(\lambda_{a b}-\lambda_{a b}^{e q}\right) \frac{\mathrm{d} t}{\tau}+\mathrm{d} \mathbf{W}_{a b}^{\lambda}
$$

where $\tau$ is the relaxation time and $\mathbf{W}_{a b}^{\lambda}$ is a Wiener process with time-uncorrelated increments satisfying:

$$
\left\langle\mathrm{d} \mathbf{W}_{a b}^{\lambda} \mathrm{d} \mathbf{W}_{a b}^{\lambda}\right\rangle=\left(\frac{2 k_{B} T}{\alpha}\right)\left(\frac{\mathrm{d} t}{\tau}\right) \mathbf{I} .
$$

The function $\lambda_{a b}^{e q}$, shorthand for $\lambda^{e q}\left(r_{a b}\right)$, is the equilibrium value of the variable $\lambda_{a b}$ for the distance $r_{a b}$ between polymers $a$ and $b$. For the definition of $\lambda_{a b}^{e q}$, we use here 
the following form that has been used in the literature: [33, 34, 35]

$$
\lambda^{e q}\left(r_{a b}\right)= \begin{cases}\left(1-\frac{r_{a b}}{r_{c}}\right)^{2} & \text { for } r_{a b} \leq r_{c} \\ 0 & \text { for } r_{a b} \geq r_{c} .\end{cases}
$$

A particularly suggestive form emphasizing the type of memory that may be incorporated into the description by this method is given in reference [36], while a simple extension of the present application is presented in reference [37].

The potential which drives the fluid particles consists of the pressure and possibly external body forces, which are absent in the present application. For the pressure we use the commonly used pseudo-incompressible equation of state given by:[38, 39]

$$
P_{i}=P_{0}\left[\left(\frac{n_{i}^{f}}{\bar{n}^{f}}\right)^{7}-1\right]
$$

where $P_{0}$ is chosen such that the velocity of sound in the simulation is sufficiently large and the density fluctuations of fluid particles are sufficiently small, in order that our fluid resembles an incompressible fluid.

\subsubsection{Weight functions and resolution}

For the polymer blobs, we have used a normalized weight function that has been used previously in the literature, where the RaPiD model has been employed[28, 33, 34, 35]. It is given by $w^{p}(r)$ :

$$
w^{p}(r)= \begin{cases}\frac{15}{2 \pi\left(r_{c}^{5}-\sigma^{5}\right)}\left(r_{c}-\sigma\right)\left(r_{c}+\sigma-2 r\right) & \text { for } r \leq \sigma \\ \frac{15}{2 \pi\left(r_{c}^{5}-\sigma^{5}\right)}\left(r-r_{c}\right)^{2} & \text { for } \sigma \leq r \leq r_{c} \\ 0 & \text { for } r \geq r_{c},\end{cases}
$$

where the cut-off $r_{c}$ is chosen as $2.5 \sigma$.

For the fluid blobs, we have chosen the normalized $M_{4}$ kernel commonly used in $\mathrm{SPH}[40]$ as the weight function. It is given by $w^{f}(r)$ :

$$
w^{f}(r)= \begin{cases}\frac{1}{4 \pi h^{3}}\left[\left(2-\frac{r}{h}\right)^{3}-4\left(1-\frac{r}{h}\right)^{3}\right] & \text { for } r \leq h \\ \frac{1}{4 \pi h^{3}}\left(2-\frac{r}{h}\right)^{3} & \text { for } h \leq r \leq 2 h \\ 0 & \text { for } r \geq 2 h\end{cases}
$$


where $h$ is what is commonly referred to as the support of the weight function, which we have chosen as $h=2 \sigma$ such that the cutoff radius $R_{c}$ for the fluid blobs is larger than the cut-off radius $r_{c}$ chosen for the polymer blobs. The support is chosen such that the weight function $w^{f}(r)$ will be able to accurately estimate the second order derivatives occurring in the equation of motion for the solvent. We have chosen the number of fluid particles such that there are on average 8 particles within a sphere of radius $h$, so there are on average about 64 neighboring fluid blobs with every given fluid blob. This is more than the minimum required for this weight function for 3-D SPH simulations.[41] As we do not need to represent any gradients to describe fluidpolymer interactions, for the latter we use the weight function $w^{p}(r)$ with a smaller cut-off $r_{c}$ instead of $w^{f}(r)$ with the larger cut-off $R_{c}$ for computational efficiency.

It is pertinent to note that the relative resolution between the polymer and the fluid, let's call it $\Re=\sigma / h$, which we have chosen as $1 / 2$ for the work presented in this chapter, is actually a very important parameter. In principle, one should choose a high enough value for the relative resolution $\mathfrak{R}$ in order to accurately mimic the draining of the solvent through the polymers. Nevertheless, in practice, if one chooses a very large value for $\mathfrak{R}$, a direct consequence would be that the fluid blobs have a really small mass and hence a much smaller time-step would be required, which would drastically affect the efficiency of the method. Furthermore, in case of studying flow through a particular geometry, naturally a much larger number of fluid blobs would be required if the same length scale of the geometry is to be studied with smaller blobs. Thus, a very high resolution leads to computational problems. On the other hand, if the value is chosen to be too small, then there would be a large number of polymers per fluid blob. This leads to a conceptual problem because then one would have to capture the fluid flow field gradient within each fluid blob and transmit it to the polymers within the blob, which will need a modification of the equation of motion for the polymers adding an extra term accounting for the gradient of the fluid flow field within the fluid blob. Thus, we have chosen the sizes of the polymer blobs not very different from that of the fluid blobs. 


\subsubsection{System parameters}

The values of the system parameters have been summarized in table 4.1. The physical properties of the solvent, i.e. the density and viscosity, have been chosen to be consistent with those of water. The solute particles can be thought of as very large polymers or micro-gel particles with a radius of $5 \mu \mathrm{m}$ and a relaxation time of $1 \mathrm{~s}$. These may qualify as very large polymers or small clusters of polymers as may occur when poly-electrolytes like the ones used in enhanced oil recovery, at particular salt concentrations and $\mathrm{pH}$ conditions, form salt bridges among each other.

Table 4.1: Summary of system parameters

\begin{tabular}{llll}
\hline \hline System parameter & Symbol & Value & Unit \\
\hline Solute length scale & $\sigma$ & 5.0 & $\mu \mathrm{m}$ \\
Friction coefficient & $\xi$ & $1.0 \times 10^{-7}$ & $\mathrm{~kg} / \mathrm{s}$ \\
Number of Kuhn segments & $\mathrm{p}$ & 300,000 & - \\
Maximum number density of polymers & $n_{\text {max }}^{p}$ & $1.0 \times 10^{4}$ & $\mathrm{C}^{*}$ \\
Flory Huggins interaction parameter & $\chi$ & 0.5 & - \\
Strength of polymer interactions & $\alpha$ & 500 & $k_{B} T$ \\
Relaxation time & $\tau$ & 1.0 & $\mathrm{~s}$ \\
Spring constant & $k$ & 50 & $k_{B} T / \sigma^{2}$ \\
\hline Solvent length scale & $h$ & 10.0 & $\mu \mathrm{m}$ \\
Resolution of fluid & $\bar{n}^{f}$ & 8 & $\mathrm{particles} / \mathrm{h}^{3}$ \\
Density of fluid & $\rho$ & 1000 & $\mathrm{~kg} / \mathrm{m}^{3}$ \\
Viscosity of fluid & $\eta$ & 1.0 & $\mathrm{mPa} . \mathrm{s}$ \\
Pressure coefficient & $P_{0}$ & 0.13 & $\mathrm{~Pa}$ \\
\hline Time step & $\mathrm{d} t$ & 10.0 & $\mu \mathrm{s}$ \\
Temperature & $T$ & 300 & $\mathrm{~K}$ \\
\hline \hline
\end{tabular}

\subsection{Results and Discussion}

We have performed shear flow simulations using the Lees-Edwards technique[42] for the model system that we described in Section 4.3 for two concentrations $-2.5 \mathrm{C}^{*}$ and $5 \mathrm{C}^{*}$. In each simulation, we apply a constant shear across the box and measure 
the shear stresses in the system. From the steady-state shear stresses, we calculate the steady-state shear viscosities for the two concentrations for different shear rates. In Fig.4.1, we summarize the results of all the shear-flow simulations in the form of flow curves for our model system at two different concentrations $-2.5 \mathrm{C}^{*}$ and $5 \mathrm{C}^{*}$.

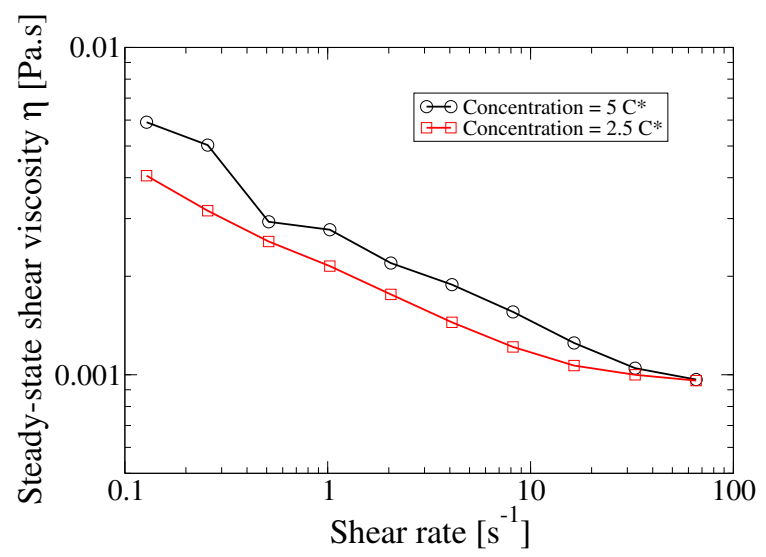

Figure 4.1: Flow curves for the two different systems calculated from shear flow simulations

\subsubsection{Shear Banding}

In the range of shear rates from about $0.1 \mathrm{~s}^{-1}$ to about $1 \mathrm{~s}^{-1}$, the system at a concentration of $5 \mathrm{C}^{*}$ exhibits shear banding. As a representative example of the shear banding that we observe, in Fig.4.2 we show the flow and stress development of the system at a shear rate of $0.26 \mathrm{~s}^{-1}$. From the top graph, it is evident that the fluid velocities of the inner slabs come together to form a band of very low shear rate, whereas the outer slabs form a band of high shear rate. Recall that the system is periodic in the gradient direction, implying that the two outer bands actually constitute one band (the slopes in the two outer bands are the same). The velocities of the polymer blobs for this low shear rate are obviously drowned in the fluctuations, which are much larger than the systematic velocities for this shear rate. But from Fig.4.3, it is evident that the bands have formed already at $50 s$ and have attained steady state, as the velocities have hardly changed till 200 s. 

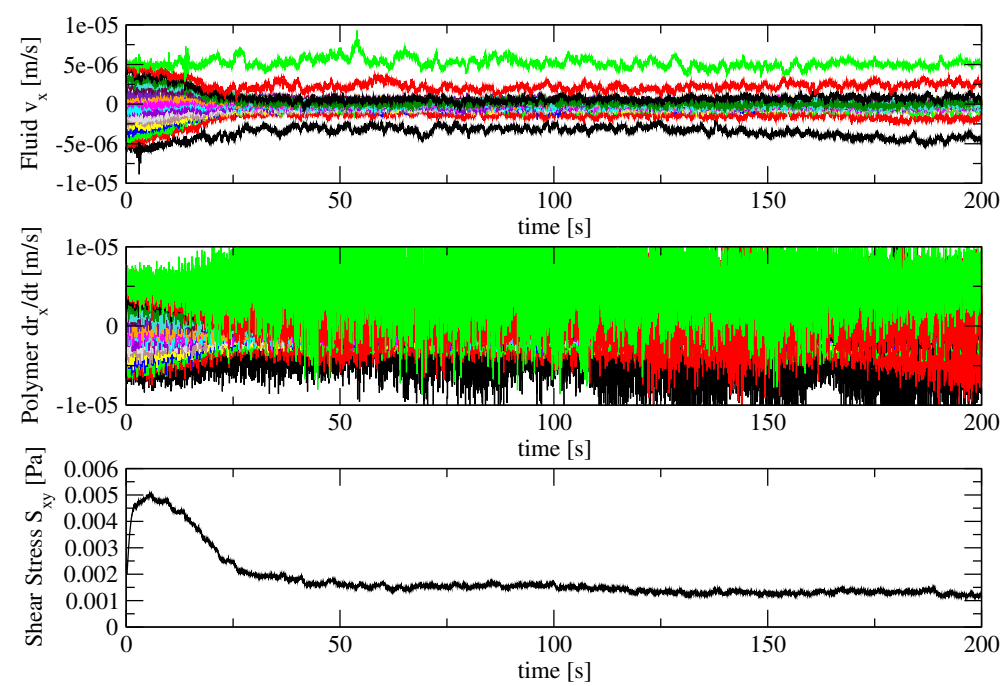

Figure 4.2: Velocity profiles of different slabs for fluid (top) and polymers (middle), and overall shear stress $S_{x y}$ (bottom) as a function of time for a concentration of $5 C^{*}$ at a shear rate of $0.26 \mathrm{~s}^{-1}$.

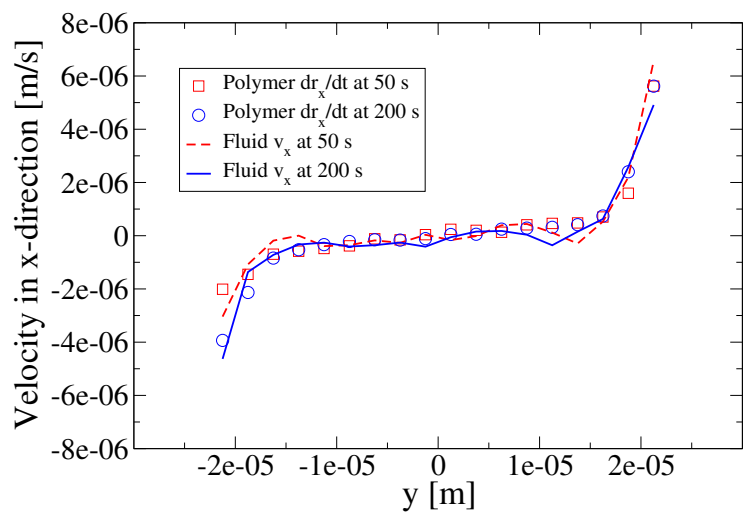

Figure 4.3: Polymer and fluid velocity profiles at two different times for a concentration of $5 C^{*}$ at a shear rate of $0.26 \mathrm{~s}^{-1}$. 
Furthermore, we show the density of the polymer and the fluid blobs across the simulation box in fig.4.4. It is clearly evident that the concentration of the polymer blobs in the low-shear-rate band is higher than in the high-shear rate region. This is a typical case of concentration-coupled shear banding. It is also clear from Fig.4.4 that the density of fluid blobs remains constant throughout the simulation box. Thus, we would like to re-emphasize that with our HCBD method, on the one hand, the fluid and the polymer are very well coupled with each other in the sense that the polymers truly follow the flow which is influenced of course by the presence of the polymers, while on the other hand, their local densities can still be different. In other words, our HCBD technique allows for cross-flow migration of the polymers in cases where the shear-rates are varying in the simulation box, as we have shown earlier as well albeit for a different case of Reverse Poiseuille Flow.[28]

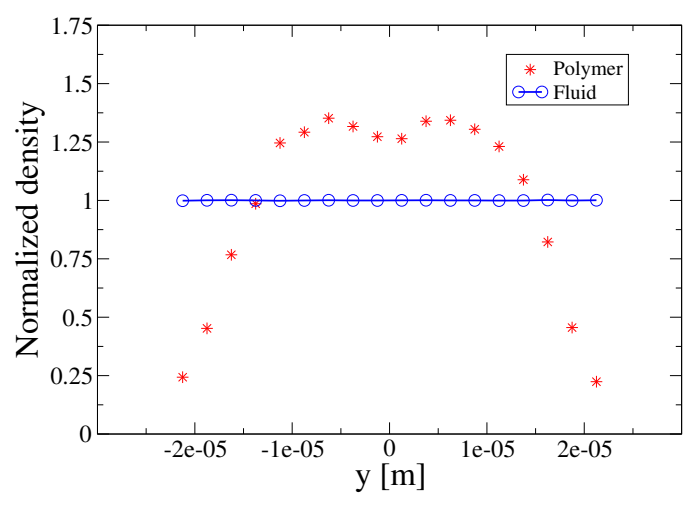

Figure 4.4: Normalized density profiles of the polymer and fluid blobs for a polymer concentration of $5 C^{*}$ at a shear rate of $0.26 \mathrm{~s}^{-1}$.

As expected, beyond some finite shear rate, in this case $16 \mathrm{~s}^{-1}$, we do not see any shear banding nor do we see any micro-structures forming (see section 4.4.2). This is surely because shear forces are too high in these cases to allow any such structures to be formed by the quasi-elastic interactions between the polymers. As a representative example, we have shown the response of the system to a shear rate of $65 \mathrm{~s}^{-1}$. From fig.4.5 and 4.6, it is clear that the polymers and the fluid have a linear velocity profile 

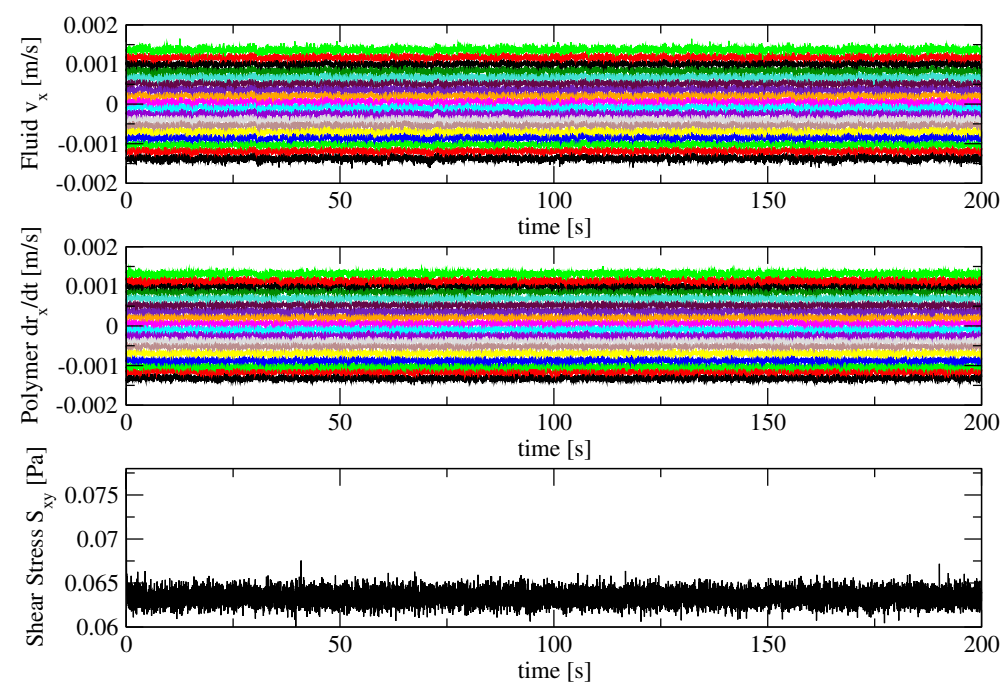

Figure 4.5: Velocity profiles of different slabs for fluid (top) and polymers (middle), and overall shear stress $S_{x y}$ (bottom) as a function of time for a concentration of $5 C^{*}$ at a shear rate of $65.5 \mathrm{~s}^{-1}$.

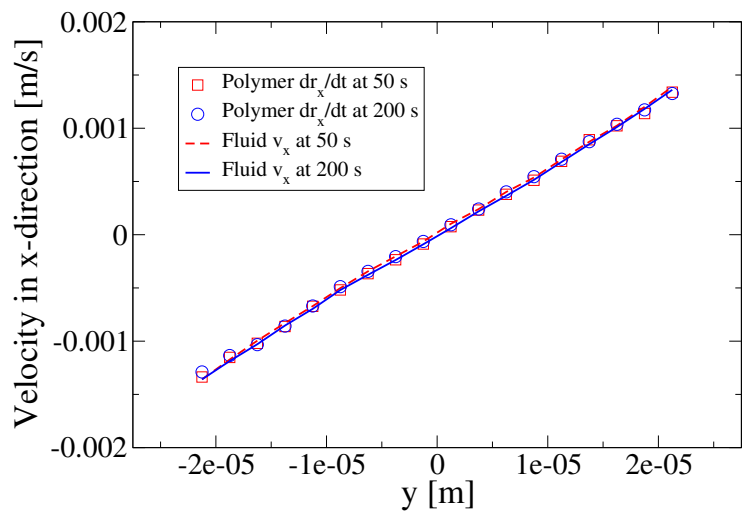

Figure 4.6: Polymer and fluid velocity profiles at two different times for a concentration of $5 C^{*}$ at a shear rate of $65.5 \mathrm{~s}^{-1}$. 
indicating that there is no shear-banding observed for this case. For lower concentration of the polymers - namely $2.5 \mathrm{C}^{*}$, we do not see shear banding as is evident from fig.4.7 and 4.8. Although the profile is still not very linear, it does not form any bands, and from fig.4.9 and 4.10, it is evident that at higher shear rates, the velocity profile becomes exactly linear.
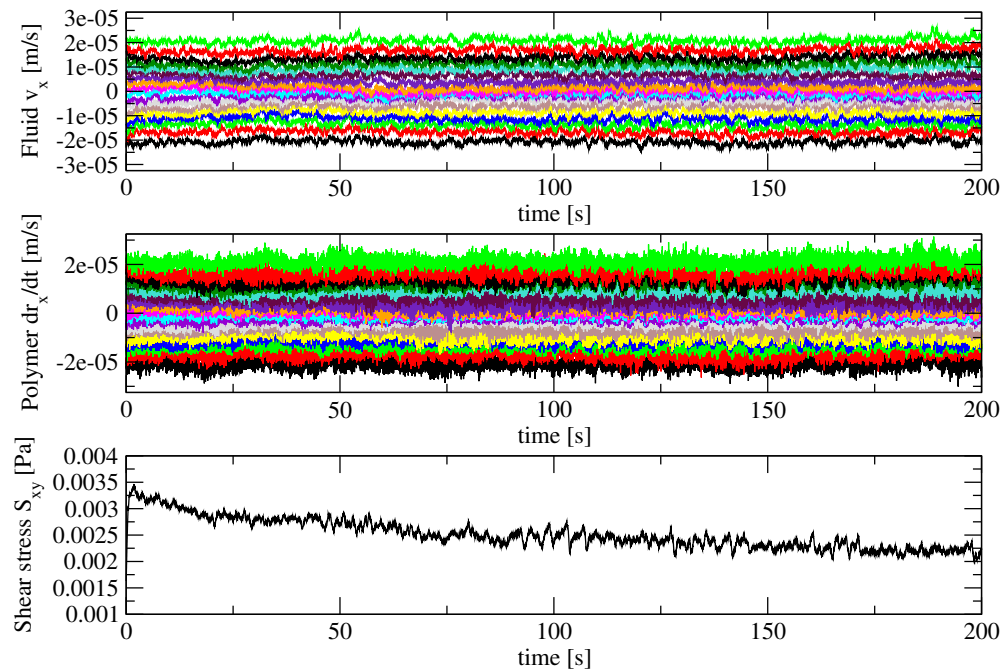

Figure 4.7: Velocity profiles of different slabs for fluid (top) and polymers (middle), and overall shear stress $S_{x y}$ (bottom) as a function of time for a concentration of $2.5 C^{*}$ at a shear rate of $1 \mathrm{~s}^{-1}$. 


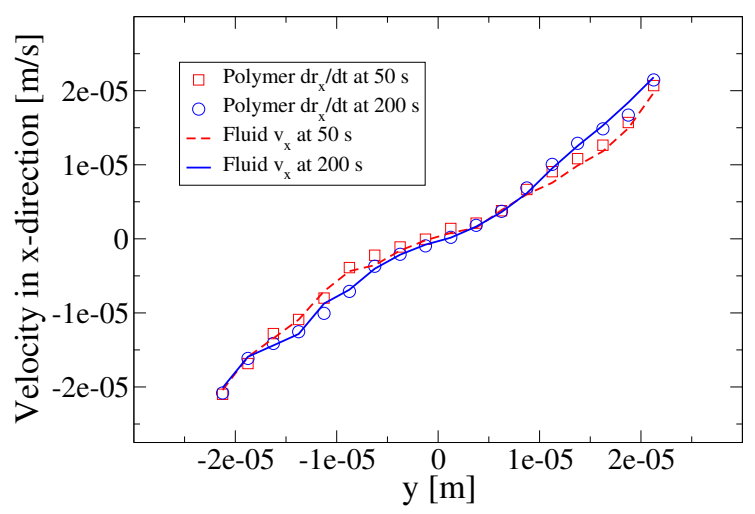

Figure 4.8: Polymer and fluid velocity profiles at two different times for a concentration of 2.5C* at a shear rate of $1 \mathrm{~s}^{-1}$.
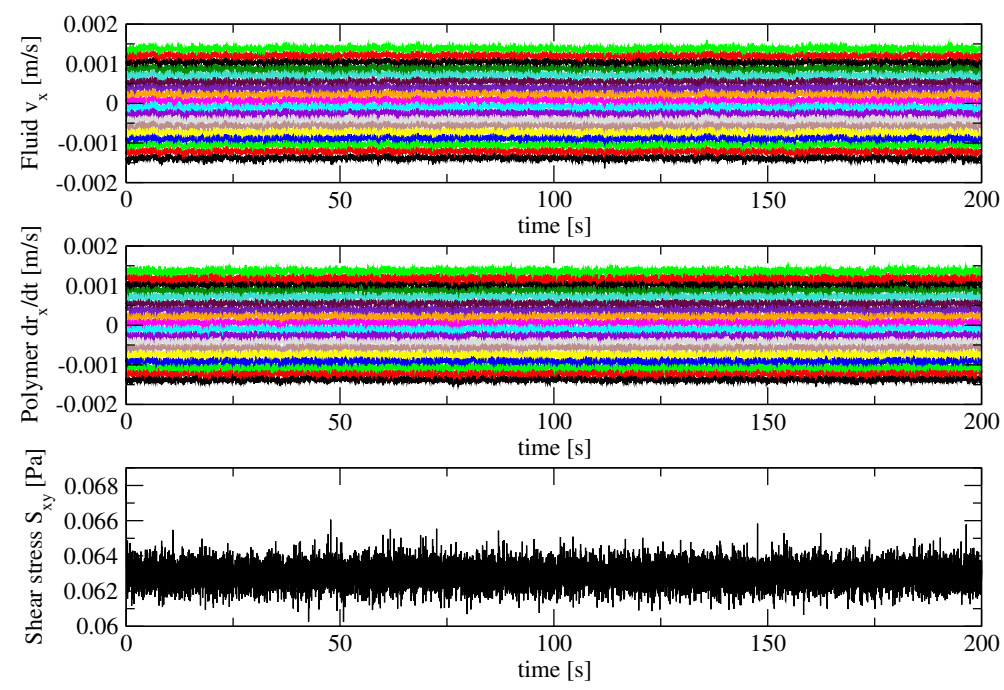

Figure 4.9: Velocity profiles of different slabs for fluid (top) and polymers (middle), and overall shear stress $S_{x y}$ (bottom) as a function of time for a concentration of $2.5 C^{*}$ at a shear rate of $65.5 \mathrm{~s}^{-1}$. 


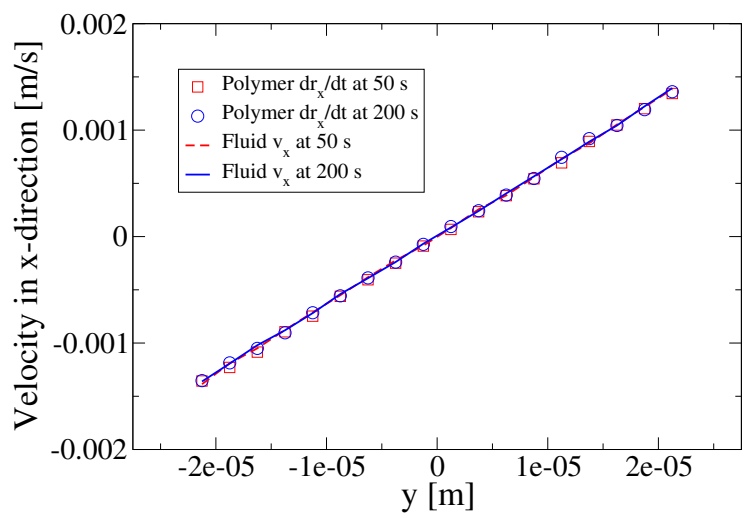

Figure 4.10: Polymer and fluid velocity profiles at two different times for a concentration of $2.5 C^{*}$ at a shear rate of $65.5 \mathrm{~s}^{-1}$.

Thus, we only observe shear banding and elastic instabilities for our model system at higher concentrations and that too only for high $\alpha$ values. Although we have not shown here a full parametric sensitivity analysis, we simulated the same system at higher concentration for lower $\alpha$ values such as 100 and below and we did not observe any shear-banding for these $\alpha$ values. This shows that the shear banding and elastic instabilities are primarily due to the quasi-elastic interactions between polymers and not the internal elasticity due to the finitely extensible non-linear elastic (FENE) springs between the two blobs of each polymer dumbbell.

\subsubsection{Shear Rolls}

Now, we come to the most interesting part of the phase space for our model system, which is at high concentration of $5 \mathrm{C}^{*}$ at shear rates in the range of $1 \mathrm{~s}^{-1}$ to $10 \mathrm{~s}^{-1}$. From the top graph in fig.4.11, i.e. from the velocity profiles of the fluid blobs, it seems as though at 100s, the shear banding has given way to homogeneous shear again. 

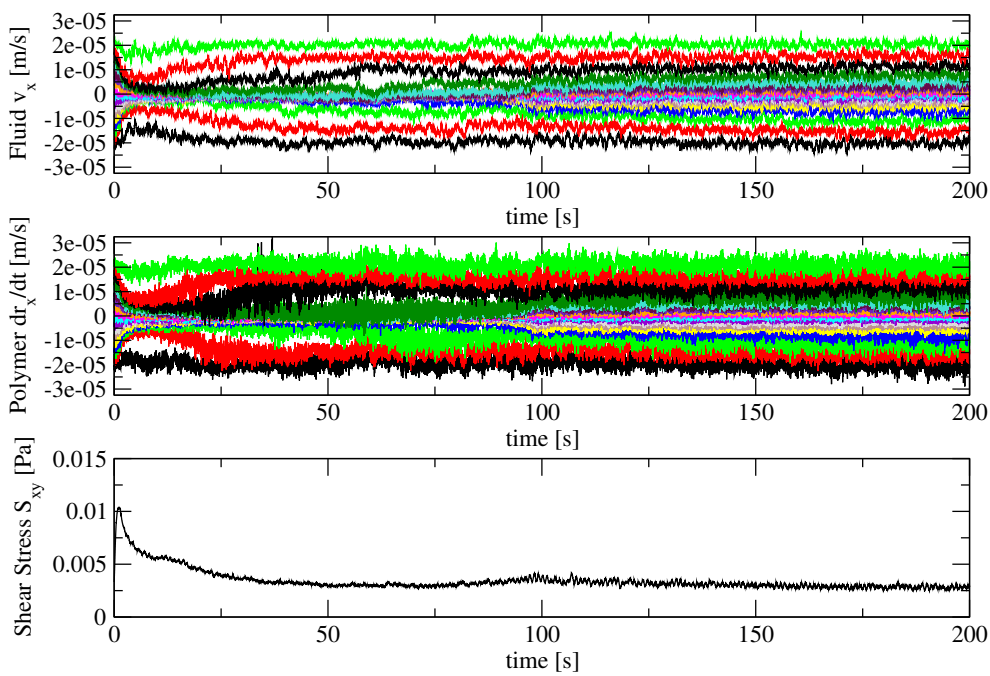

Figure 4.11: Velocity profiles of different slabs for fluid (top) and polymers (middle), and overall shear stress $S_{x y}$ (bottom) as a function of time for a concentration of $5 C^{*}$ at a shear rate of $1 \mathrm{~s}^{-1}$.

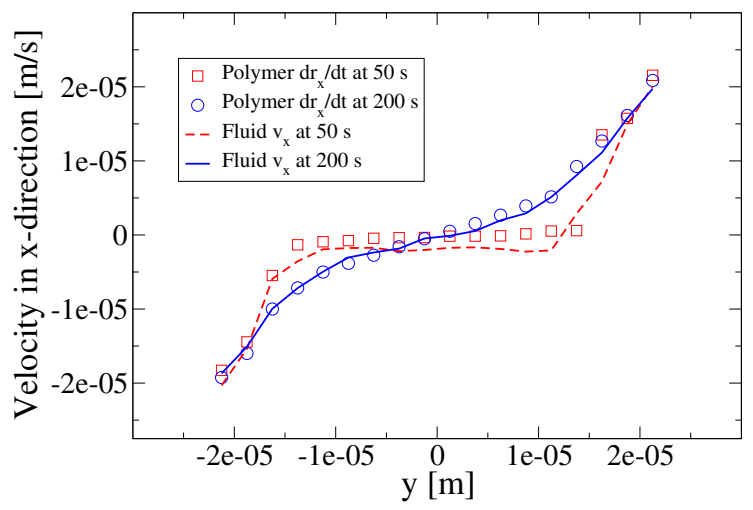

Figure 4.12: Polymer and fluid velocity profiles at two different times for a concentration of 5C* at a shear rate of $1 \mathrm{~s}^{-1}$. 
However, a closer look at the distribution of polymers in this case reveals that it is actually the result of the presence of a rotating cylinder shown in fig.4.13(a), which has formed due to a quasi-elastic instability.

The cylinder undergoes an almost solid-like rotation because the shear from the top and bottom layers provides the necessary torque for the rotation. The formation and rotation of this cylinder can be seen by visual inspection of the movie "SR_C5_g1.mpg" added in supplementary material.

The quasi-elastic instability actually occurs for our model system for a range of shear rates from about $1 \mathrm{~s}^{-1}$ to about $10 \mathrm{~s}^{-1}$ in various forms. Examples of these types of elastic instabilities such as spontaneous formation of micro-structures like cylinders, spirals and streaks for different shear rates are shown in fig.4.13. At shear rates of $1 \mathrm{~s}^{-1}$ and $2 \mathrm{~s}^{-1}$, we see cylindrical structures forming but at higher shear rates of $4 \mathrm{~s}^{-1}$, these cylinders get sheared due to the high shear rates. At even higher shear rates of $8 s^{-1}$, the shear rate is too high for cylinders to form but still some ring-like structures form once in a while and are sheared later into streaks.

We must further emphasize that these structures are primarily forming because of the interactions between the polymers due to quasi-elastic forces occurring in our model through the transient potentials of the RaPiD technique as we have checked that no such instabilities occur in case $\alpha$ is chosen very small. 


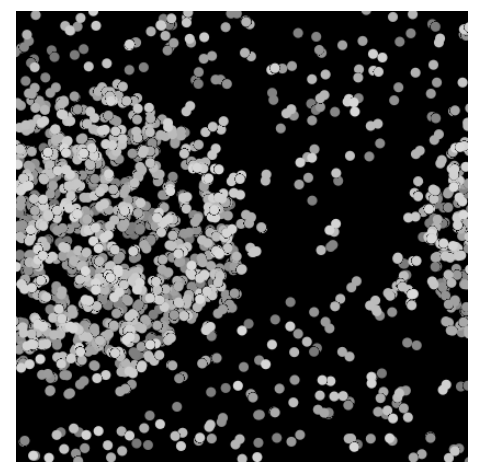

(a) Shear rate $=1 \mathrm{~s}^{-1}$

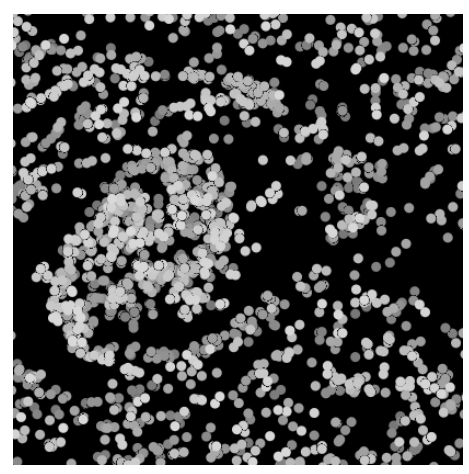

(c) Shear rate $=4 \mathrm{~s}^{-1}$

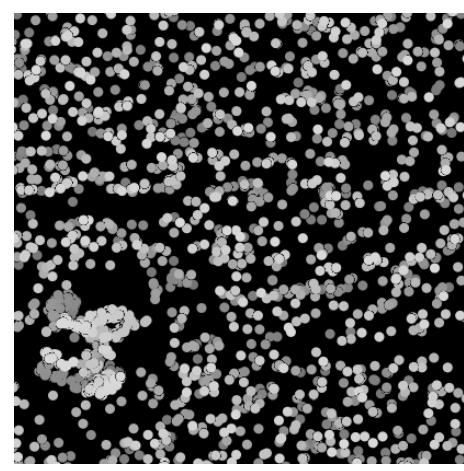

(e) Shear rate $=8 \mathrm{~s}^{-1}$

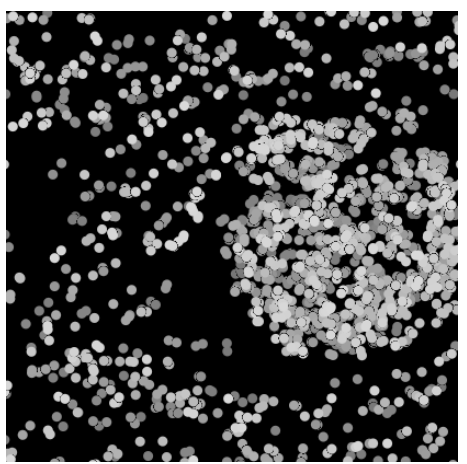

(b) Shear rate $=2 \mathrm{~s}^{-1}$

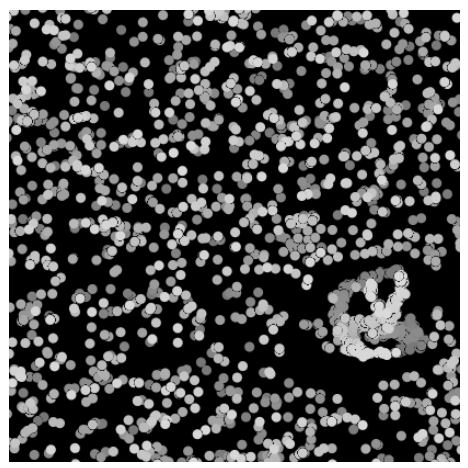

(d) Shear rate $=8 \mathrm{~s}^{-1}$

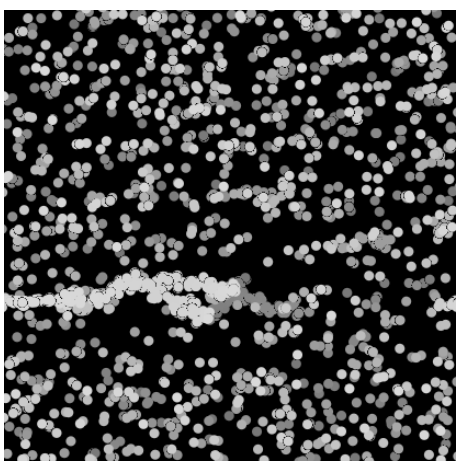

(f) Shear rate $=8 \mathrm{~s}^{-1}$

Figure 4.13: Microstructures formed by polymers due to quasi-elastic instabilities observed for a polymer concentration of $5 C^{*}$ in the range of shear rates between $1 \mathrm{~s}^{-1}$ and $10 \mathrm{~s}^{-1}$. 


\subsection{Conclusion and scope for further research}

In this chapter we have studied the rheological properties for two concentrations of our model polymer solution using our recently developed Hydrodynamically Coupled Brownian Dynamics method. In this method the motion of the polymers is coupled to that of a solvent, represented by fluid blobs according to Smooth Particle Hydrodynamics, in a consistent momentum conserving way. An important ingredient of the HCBD method is that flows can develop naturally without being forced into a background 'template' like for example in the SLLOD simulation method. This enhanced flexibility of the code allows for more complex flows than possible with SLLOD or with a previous Brownian dynamics simulation method in which gradients of shear rates were allowed only along the shear gradient direction. Velocity gradients act on the polymers through friction forces at the positions of two beads constituting the polymer. A spring of finite maximum extension keeps the two beads together. Memory effects mimicking the resistance of two overlapping polymers to separate are introduced by so-called transient forces.

For smaller concentrations, we do not find shear banding but rather just a simple shear thinning profile predicted by the simplest theories, and similar to the ones obtained previously in simple Brownian dynamics simulations without hydrodynamic coupling. With the larger concentration, we not only find shear banding, but also additional instabilities within the bands caused by the quasi-elastic transient forces. It will be interesting to systematically investigate the latter instabilities in more detail, and by this setting a challenge for theoreticians to develop constitutive models flexible enough to predict similar phenomena. These theories then should also include the possibility of developing large concentration gradients in the system. Some recent work along this direction has been done by Dhont et al. [44, 45] 


\section{Bibliography}

[1] N. A. Spenly, M. E. Cates, and T. C. B. McLeish, Physical Review Letters, 71, 939 (1993).

[2] J. F. Berret, G. Porte G, and J. P. Decruppe, Physical Review E, 55, 1668 (1997).

[3] J. F. Berret, D. C. Roux, and P. Lindner, European Physics Journal B, 5, 67 (1998).

[4] M. M. Britton and P. T. Callaghan, European Physics Journal B, 7, 237 (1999).

[5] P. T. Callaghan and A. M. Gil, Macromolecules, 33, 4116 (2000).

[6] D. Tapadia, S. Ravindranath, and S. Q. Wang, Physical Review Letters, 96, 19600 (2006).

[7] M. Cromer, G. H. Fredrickson, and G. H. Leal, Physics of Fluids, 26, 063101 (2014).

[8] J. van der Gucht, M. Lemmens, W. Knoben, N. A. M. Besseling, and M. P. Lettinga, Physical Review Letters, 97, 108301 (2006).

[9] P. D. Olmsted, Current Opinions in Colloid and Interface Science, 4, 95 (1999).

[10] J. Vermant, Current Opinions in Colloid and Interface Science, 6, 489 (2003).

[11] M. E. Cates and S. M. Fielding, Advances in Physics, 55, 799 (2006).

[12] S. M. Fielding, Soft Matter, 3, 1262 (2007).

[13] J. K. G. Dhont and W. J. Briels, Rheologica Acta, 47, 257 (2008).

[14] P. D. Olmsted, Rheologica Acta, 47, 283 (2008).

[15] S. Manneville, Rheologica Acta, 47, 301 (2008).

[16] T. Divoux, M. A. Fardin, S. Manneville, and S. Lerouge, Annual Review of Fluid Mechanics, 48, 81, (2016). 
[17] J. K. G. Dhont, Physical Review E, 60, 4534 (1999).

[18] C. Y. D. Lu, P. D. Olmsted, and R. C. Ball, Physical Review Letters, 84, 642 (2000).

[19] A. W. El-Kareh and L. G. Leal, Journal of Non-Newtonian Fluid Mechanics, 33, 257 (1989).

[20] M. Mohagheghi and B. Khomami, ACS Macro Letters, 4, 684 (2015).

[21] A. van den Noort, W. K. den Otter, and W. J. Briels, Europhysics Letters, 80, 28003 (2007).

[22] W. J. Briels. Soft Matter, 5, 4401 (2009).

[23] A. van den Noort and W. J. Briels, Macromolecular Theory and Simulations, 16, 742 (2007).

[24] A. van den Noort and W. J. Briels, Journal of Non-Newtonian Fluid Mechanics, 152, 148 (2008).

[25] J. Sprakel, J. T. Padding, and W. J. Briels, Europhysics Letters, 93, 58003 (2011).

[26] D. J. Evens and G. P. Morris, Statistical Mechanics of Nonequilibrium Liquids (Academic Press, 1990).

[27] J. F. Brady and G. Bossis, Annual Review of Fluid Mechanics, 20, 111 (1988).

[28] V. R. Ahuja, J. van der Gucht, and W. J. Briels, The Journal of Chemical Physics, 148, 034902 (2018).

[29] L. D. Landau and E. M. Lifshitz, Fluid Mechanics (Pergamon Press, 1959).

[30] L. D. Landau and E. M. Lifshitz, Statistical Physics (Pergamon Press, 1958).

[31] J. J. Monaghan, Annual Review of Astronomy and Astrophysics, 30, 543 (1992). 
[32] A. Vázquez-Quesada, M. Ellero, and P. Español, The Journal of Chemical Physics, 130, 034901 (2009).

[33] I. S. Santos de Oliveira, A. van den Noort, J. T. Padding, W. K. den Otter, and W. J. Briels, The Journal of Chemical Physics, 135, 104902 (2011).

[34] I. S. Santos de Oliveira, W. K. den Otter, and W. J. Briels, The Journal of Chemical Physics, 137, 204908 (2012).

[35] I. S. Santos de Oliveira, B. W. Fitzgerald, W. K. den Otter, and W. J. Briels, The Journal of Chemical Physics, 140, 104903, (2014).

[36] W. J. Briels. In Computational Trends in Solvation and Transport in Liquids, 557-574. (Springer, 2015).

[37] B. W. Fitzgerald and W. J. Briels, Macromolecular Theory and Simulations, 27, 1700069 (2018).

[38] G. K. Batchelor. An Introduction to Fluid Dynamics (Cambridge university press, 2000).

[39] R. H. Cole, Underwater explosions (Dover Publications, 1965).

[40] J. J. Monaghan, Reports on Progress in Physics, 68, 1703 (2005).

[41] A. P. Whitworth, A. S. Bhattal, J. A. Turner, and S. J. Watkins, Astronomy and Astrophysics, 301, 929 (1995).

[42] A. W. Lees and S. F. Edwards, Journal of Physics C: Solid State Physics, 5, 1921 (1972).

[43] M. Cloitre, R. Borrega, F. Monti, and L. Leibler, Physical Review Letters, 90, 068303 (2003).

[44] H. Jin, K. Kang, K. H. Ahn, and J. K. G. Dhont, Soft Matter, 10, 9470 (2014).

[45] J. K. G. Dhont, K. Kang, H. Kriegs, O. Danko, J. Marakis, and D. Vlassopoulos, Physical Review Fluids, 2, 043301 (2017). 



\section{Flow through porous media}

We present coarse-grain particle-based simulations for studying flow of complex soft matter systems such as solutions of large polymers or micro-gel particles through model porous media. Both the solute and solvent particles are modeled using mesoscopic blobs. However, as opposed to the rigid spherical solvent blobs, the solute blobs are deformable as they are modeled using Finitely Extensible Non-linear Elastic (FENE) springs. We use our recently published two-way coupling technique called Hydrodynamically Coupled Brownian Dynamics (HCBD) for coupling the solute particles to the solvent [J. Chem. Phys. 148, 034902 (2018)]. In this work, we study the flow of such systems through model porous media, which are constructed using periodic arrays of solid beams arranged on a square grid, the plane of which is perpendicular to the axes of the beams. For applying the no-slip condition, we use artificial particles embedded within the solid beams. In the current work, we have studied the flow of the model polymer solution across two different types of beams ones having a circular cross-section which we call cylindrical beams and another ones having a square cross-section which we call cuboidal beams. We study two extreme angles of attack - one parallel to one of the cartesian directions of the plane of the square grid of beams and another at an equal angle ( 45 degrees) from either cartesian directions of the same plane. Thus we study the four different cases - two geometries and two flow directions. We compare the results with the flow of pure Newtonian solvent, which we have simulated using standard Smoothed Particle Hydrodynamics (SPH) simulations. We observe that in all these cases of flow through porous media, the concentration of polymers at steady state is not uniform even though we start the simulation with a uniform polymer concentration. This is indicative of shear-induced cross-flow migration, that we have observed for such systems in the bulk shear flow simulations in our earlier work. Furthermore, we see the characteristic flattening of the velocity profile experimentally observed for a shear-thinning polymer solution flow through channels as opposed to the parabolic Poiseuille flow profile for Newtonian fluid flow through a similar geometry. 


\subsection{Introduction}

After several years of oil recovery, many oil reservoirs have become 'mature' and are consequently seeing a decline in production. This however does not imply that the oil in these reservoirs has been completely recovered. The recovery ratio of these socalled 'matured' oil reservoirs is still 15 to $35 \%$ of the original oil in place (OOIP),[1] implying that there is still a substantial production potential in these reservoirs. The obvious question that arises next is why this substantial unexplored potential is not tapped into. The reason is that it is substantially more expensive to recover the residual oil in these reservoirs. For understanding why this is the case, it is important to understand the different stages of oil recovery from an oil reservoir, which we shall briefly explain.

The first stage of oil recovery from an oil reservoir known as primary recovery typically involves the digging of a production well or wells, through which the oil naturally comes out of the reservoir due to the high pressure of the oil in the reservoir and simultaneous rock decompression. This results in the recovery of 5 to $25 \%$ of OOIP depending on the type of oil reservoir. Light oils typically respond well to primary recovery whereas heavy oils and particularly tar sands respond very poorly.[2] Actually, the same can be said about their response to the second stage of oil recovery known as secondary recovery. This stage involves drilling additional wells known as injection wells, through which a driving fluid such as water or steam is pumped into the reservoir, which leads to an additional recovery of 5 to $30 \%$ of OOIP depending on the type of oil reservoir. Thus, the most conservative estimate of the oil still remaining in the reservoir after primary and secondary recovery is at least $45 \%$ of OOIP and practically in many cases it is significantly more than $50 \%$ of OOIP up to almost $70 \%$ of OOIP in some cases.[3] This illustrates the need for a tertiary stage of oil recovery, which is typically done by one of the many processes falling under the heading of Enhanced Oil Recovery (EOR) operations, where the objective is to recover a part of this residual oil, albeit at a higher cost. The economic feasibility of EOR operations obviously rests, among other things, on the balance between the 
additional cost of recovering this residual oil $v s$. the additional revenue of selling this oil at the existing market price at the time of sale.

Before going into the details of Enhanced Oil Recovery (EOR) operations, it is important to understand the reasons why the remaining oil is still trapped in the reservoir after the primary and secondary recovery. The main reasons are either that the oil is trapped in the reservoir due to capillary forces or that the driving fluid is bypassing the oil due to differences in mobility between the two fluids. $[1,4]$ There are thus two dimensionless ratios $v i z$. the capillary number $N_{c}$ and the mobility ratio $M$, which are considered most important in EOR as they primarily influence the mobilization of oil through the reservoir.[2] The capillary number $N_{c}$ is defined as $v \mu / \sigma$, where $v$ is the Darcy velocity, $\mu$ is the viscosity of the displacing fluid and $\sigma$ is the interfacial tension between the displacing fluid and the oil in the reservoir. The mobility ratio $M$, on the other hand, is defined as $\lambda_{d} / \lambda_{o}$, where $\lambda_{d}$ is the mobility of the displacing fluid and $\lambda_{o}$ is the mobility of the oil, i.e. the displaced fluid. The mobility of each fluid is essentially a ratio of its permeability through the reservoir to its viscosity. Thus, to recover more oil, one must increase the capillary number and / or reduce the mobility ratio. In other words, one might say that loosely speaking one must try to make the displacing fluid 'like' the oil or vice versa (pun intended).

Thermal EOR methods, typically involving steam, are best suited for heavy oils (10-20 $\mathrm{API})$ and tar sands $\left(\leq 10^{\circ} \mathrm{API}\right)$ as they reduce the mobility ratio by reducing the viscosity of the oil bringing it closer to the displacing fluid, thereby preventing bypassing of the displacing fluid. ${ }^{1}$ Non thermal methods like chemical injection either improve the capillary number by reducing the interfacial tension by adding a surfactant to the displacing fluid for instance or reduce the mobility ratio by adding polymers to the displacing fluid increasing its viscosity and bringing it closer to the oil. Furthermore, addition of polymers to the displacing fluid also introduces viscoelasticity and may lead to elastic instabilities which can further improve the oil recovery. It is this viscoelastic effect of the polymers that we wish to capture with our

\footnotetext{
${ }^{1}$ The American Petroleum Institute gravity of a fluid with specific gravity SG is defined as: ${ }^{\circ} \mathrm{API}=\frac{141.5}{\mathrm{SG}}-131.5$. It essentially indicates how different the density of a petroleum liquid is compared to that of water. If ${ }^{\circ} \mathrm{API}$ gravity of a fluid is greater than 10 , it is lighter than water and if less than 10 , it is heavier than water.
} 
model, thereby improving the understanding of this polymer flooding operation, in which polymers are added to the displacing fluid and the solution is pumped through the reservoir to displace more oil.

The viscoelastic flow of polymer solutions through porous media has been studied using experiments as well as simulations. Experimentally, viscoelastic flow of fluids such as polymer solutions through porous media such as packed beds, periodic arrays of cylinders, even sandstone microfluidic devices mimicking oil reservoirs etc. have been studied.[3, 6, 7, 8, 9, 10, 11, 12] On the simulation front, continuum models based on finite-element or finite-volume method have been frequently used to study the flow of polymer solutions through porous media.[13, 14, 15, 16, 17] However, these methods do not fundamentally bridge the gap between molecular level interactions with the macroscopic flow as they do not explicitly model the motion of polymers but rather use constitutive equations for calculating the divergence of the nonNewtonian part of the stress tensor. Our objective in this chapter is to study the flow of polymer solutions through porous media using simulations that do not assume any constitutive relations but rather let the stresses emerge from the molecular level interactions based on the positions of coarse-grained polymers. This provides a greater understanding of how the microstructures formed due to molecular level interactions are related to the macroscopic flow. Furthermore, it provides a handle for modifying the interactions on the molecular level and seeing its effect on the macroscopic flow phenomena. In this initial work however, we mainly provide a proof of concept of how a technique that bridges the Brownian motion of the polymers with the hydrodynamic flow can be successfully applied to study flow of polymer solutions through porous media so that further work in this manner can be performed to better elucidate the effect of molecular-level interactions on macroscopic flow phenomena.

The solute polymer blobs are represented in our model as deformable mesoscopic blobs such that each solute blob is actually made up of two lobes or blobs connected to each other with a Finitely Extensible Non-linear Elastic (FENE) spring. The solvent is also represented explicitly with mesoscopic blobs, albeit non-deformable ones made up of a single particle centered at the center-of-mass position of the fluid 
blob. To couple the solute with the solvent, we have used our recently published two-way coupling technique called Hydrodynamically Coupled Brownian Dynamics (HCBD).[18] In chapter 4, we have shown that such systems made up of large mesoscopic polymer blobs exhibit cross-flow migration and elastic instabilities under shear. In that work, we also described the shear thinning behavior of such systems by performing shear flow simulations in the bulk using the Lees-Edwards method.[19] In this work, we study the flow of such systems through model porous media, which is constructed using periodic arrays of solid beams arranged on a square grid, the plane of which is perpendicular to the axes of the beams. For applying the no-slip condition, we use artificial particles embedded within the solid beams as we have done in another previous work.[20] In the current work, we study the flow of our model polymer solution across two different types of beams - ones with a circular cross-section which we call cylindrical beams and another ones having a square cross-section which we call cuboidal beams. We study two extreme angles of attack - one parallel to one of the cartesian directions of the plane of the square grid of beams and another at an equal angle (45 degrees) from either cartesian directions of the same plane. Thus we study four different cases - two geometries and two flow directions. We compare the results with the flow of pure Newtonian solvent, which we have simulated using standard Smoothed Particle Hydrodynamics (SPH) simulations.

\subsection{Method}

\subsubsection{Equation of motion for the polymer blobs}

Consider the motion of a mesoscopic particle, viz. a large polymer molecule or a micro-gel particle, represented with a deformable blob made up of two mesoscopic blobs connected to each other with a finitely extensible non-linear elastic spring present in a flowing solvent. The position of the center-of-mass of any given blob (or lobe) $a$ can be updated as follows:

$$
\mathrm{d} \mathbf{r}_{a}=\mathbf{v}\left(\mathbf{r}_{a}\right) \mathrm{d} t+\left(\frac{\mathbf{F}_{a}}{\xi_{a}}\right) \mathrm{d} t+k_{B} T \frac{\partial}{\partial \mathbf{r}_{a}}\left(\frac{1}{\xi_{a}}\right) \mathrm{d} t+\mathrm{d} \mathbf{W}_{a}^{R} .
$$


Here, $\mathbf{r}_{a}$, shorthand for $\mathbf{r}_{a}(t)$, is the position of mesoscopic blob $a$ at time $t$ and $\mathbf{v}\left(\mathbf{r}_{a}\right)$, shorthand for $\mathbf{v}\left(\mathbf{r}_{a}, t\right)$, is the background fluid velocity at the position of the blob at that time, $\mathrm{d} t$ is the time-step and $\mathbf{F}_{a}$, shorthand for $\mathbf{F}_{a}(t)$, is the driving force acting on blob $a$ as a result of the interaction with other such blobs, in addition to any force field that may have been applied. Hereafter in this chapter, we will not include the $t$ in our notation, tacitly assuming that it is implicitly present. Furthermore, $\xi_{a}$, shorthand for $\xi\left(\mathbf{r}_{a}\right)$, is the friction coefficient at the position of polymer blob $a$ and the third term on the right hand side of eq.(5.1) is a drift term accounting for the spatial variation of the friction coefficient, which we have neglected in this study as we have assumed a constant friction coefficient for the sake of simplicity. The last term on the right hand side of eq.(5.1), i.e. $\mathrm{d}_{a}^{R}$, is a random displacement typical of Brownian dynamics simulations. This random displacement is uncorrelated in time and has a magnitude that is calculated in accordance with the fluctuation dissipation theorem, satisfying:

$$
\left\langle\mathrm{d}_{a}^{R} \mathrm{~d} \mathbf{W}_{b}^{R}\right\rangle=2 k_{B} T\left(\frac{\mathrm{d} t}{\xi_{a}}\right) \delta_{a b} \mathbf{I} .
$$

The background fluid velocity at the position of the blob a i.e. $\mathbf{v}\left(\mathbf{r}_{a}\right)$ is interpolated based on the velocities of the fluid blobs in the vicinity using an appropriately normalized weight function $w^{f}(r)$ as shown below:

$$
\mathbf{v}\left(\mathbf{r}_{a}\right)=\sum_{i=1}^{N_{f}} \frac{w^{f}\left(r_{a i}\right)}{n_{i}^{f}} \mathbf{v}_{i}
$$

where $n_{i}^{f}$ is the local number density of fluid blobs calculated as $\sum_{i=1}^{N_{f}} w^{f}\left(r_{a i}\right)$.

Based on the RaPiD polymer model for FENE dumbells, the force $\mathbf{F}_{a}$ can be expressed as a sum of three terms as shown below:

$$
\mathbf{F}_{a}=-\frac{\partial}{\partial \mathbf{r}_{a}}\left[\Phi_{c}+\Phi_{t}+\Phi_{f}\right]
$$

where $\Phi_{c}$ is the so-called 'conservative' potential, $\Phi_{t}$ is the so-called 'transient' potential and $\Phi_{f}$ is the FENE potential, all of which shall be defined and described in section 5.3. 


\subsubsection{Equation of motion for the fluid blobs}

Consider a fluid blob $i$ naturally moving with the background flow field at its position of its center-of-mass. The position of its center-of-mass is thus updated using:

$$
\mathrm{d} \mathbf{r}_{i}=\mathbf{v}_{i} \mathrm{~d} t
$$

For calculating the background flow field, we discretize a modified version of the Navier-Stokes equation as described in our previous work where we have introduced an additional coupling term as part of the HCBD two-way coupling technique. [18]. Thus, we arrive at the following equation that we have used in our simulations to update the velocities of any given fluid blob $i$ :

$$
\begin{aligned}
\mathrm{d} \mathbf{v}_{i}= & -\frac{\mathrm{d} t}{m}\left[\sum_{j=1}^{N_{f}}\left(\frac{P_{i}}{\left(n_{i}^{f}\right)^{2}}+\frac{P_{j}}{\left(n_{j}^{f}\right)^{2}}\right) \frac{\mathrm{d} w^{f}}{\mathrm{~d} r}\left(r_{i j}\right) \frac{\mathbf{r}_{i j}}{r_{i j}}+\sum_{j=1}^{N_{f}} f_{i j} \mathbf{v}_{i j}\right]+\mathbf{g}_{i} \mathrm{~d} t \\
& +\frac{\mathrm{d} t}{m}\left[\sum_{a=1}^{N_{b}} \frac{w^{f}\left(r_{a i}\right)}{n_{i}^{f}} \mathbf{F}_{a}\right]+\sum_{j=1}^{N_{f}} \mathrm{~d} \mathbf{W}_{i j}^{v},
\end{aligned}
$$

where $m$ is the mass of the fluid blob $i, P_{i}$ is the pressure at the position of the fluid blob $i, n_{i}^{f}$ is the local number density of fluid blobs, $N_{b}$ is the number of polymer blobs (which is twice the number of polymers $N_{p}$ as each polymer is represented by two blobs) and $f_{i j}$, shorthand for $f\left(r_{i j}\right)$, is a symmetric function defined as follows:

$$
f\left(r_{i j}\right)= \begin{cases}-\left(\frac{2 \eta}{n_{i}^{f} n_{j}^{f}}\right) \frac{1}{r_{i j}} \frac{\mathrm{d} w^{f}}{\mathrm{~d} r}\left(r_{i j}\right) & \text { for } r_{i j} \leq R_{c} \\ 0 & \text { for } r_{i j} \geq R_{c}\end{cases}
$$

$\eta$ is the solvent viscosity, $\mathbf{v}_{i j}$ is the velocity of fluid blob $i$ relative to that of $j$ and $\mathbf{g}_{i}$ is the acceleration due to body forces. The pairwise velocity fluctuation terms are uncorrelated in time and have been calculated in an anti-symmetric manner such that $\mathrm{d} \mathbf{W}_{i j}^{v}=-\mathrm{d} \mathbf{W}_{j i}^{v}$ to conserve momentum. The properties of these momentum fluctuations $d \mathbf{W}_{i j}$ have been calculated in a way that the steady state probability distribution of the positions and velocities of the fluid in a quiescent state yields the expected equilibrium distribution, more details about which can be found in our recently published 
work.[18] Thus, we have:

$$
\begin{aligned}
\left\langle\mathrm{d} \mathbf{W}_{i j}^{v} \mathrm{~d} \mathbf{W}_{i j}^{v}\right\rangle & =\left(\frac{2 k_{B} T}{m}\right)\left(\frac{\mathrm{d} t}{m}\right) f_{i j} \mathbf{I}, \\
\left\langle\mathrm{d}^{v} \mathbf{W}_{i k}^{v} \mathbf{W}_{j l}^{v}\right\rangle & =\mathbf{0} \quad(i k \neq j l \wedge i k \neq l j) .
\end{aligned}
$$

However for computational reasons, we have ignored the fluctuations of the fluid for the simulations that we have presented in this chapter. But in principle, if one would like to see the effect of fluctuations of the fluid, one may add the additional fluctuation terms mentioned above.

\subsection{Test System and Parameters}

\subsubsection{The conservative potential}

For the conservative potential, we treat the polymer as a single entity and calculate everything needed for the potential from the center-of-mass of the polymer instead of the two beads or lobes of the polymer dumbell. We have used the Flory-Huggins potential, which has been used in the past for describing the conservative part of the interaction potential for polymer solutions[18, 21, 22, 23], defined as follows:

$$
\Phi_{c}=p k_{B} T \sum_{a=1}^{N_{p}}\left[\left(\frac{1-\phi_{a}}{\phi_{a}}\right) \ln \left(1-\phi_{a}\right)-\chi \phi_{a}\right] \text {. }
$$

Here, $N_{p}$ is the total number of polymers in solution as mentioned before, $\Phi_{c}$ is the conservative potential, $p$ is the number of Kuhn segments per polymer, $\chi$ is the solvent interaction parameter and $\phi_{a}$ is the local volume fraction of polymer blobs in the neighborhood of polymer blob $a$ at time $t$ calculated as:

$$
\phi_{a}=\frac{n_{a}^{p}}{n_{\max }^{p}} .
$$

Here, $n_{\text {max }}^{p}$ is the maximum number density of polymers that the system is allowed to reach, i.e. the melt density and $n_{a}^{p}$ is the local number density at the position of the center-of-mass of polymer blob $a$ calculated as:

$$
n_{a}^{p}=\sum_{b=1}^{N_{p}} w^{p}\left(r_{a b}\right)
$$


where $r_{a b}$ is the distance between the centers-of-mass of polymers $a$ and $b$ at time $t$ and $w^{p}(r)$ is a normalized weight function with a cut-off $r_{c}$. The superscript $p$ in $w^{p}(r)$ indicates that this is the weight function used for the polymer blobs.

\subsubsection{The transient potential}

We have used the transient potential of the RaPiD model to incorporate memory effects into the simulation model. This potential which essentially takes into account the history of the interacting polymer blobs by keeping track of dynamic variables is given by:[24, 25]

$$
\Phi_{t}=\frac{1}{2} \alpha \sum_{a, b=1}^{N_{b}}\left(\lambda_{a b}-\lambda_{a b}^{e q}\right)^{2}
$$

Here, $\Phi_{t}$ the transient potential, $\alpha$ is a parameter associated with the strength of the interactions or in other words the penalty for the deviation of the dynamic variable $\lambda_{a b}$ from its equilibrium value $\lambda_{a b}^{e q}$, shorthand for $\lambda^{e q}\left(r_{a b}\right)$. We use here the following form for $\lambda^{e q}\left(r_{a b}\right)$ that has been used earlier in the literature: [21, 22, 23]

$$
\lambda^{e q}\left(r_{a b}\right)= \begin{cases}\left(1-\frac{r_{a b}}{r_{c}}\right)^{2} & \text { for } r_{a b} \leq r_{c} \\ 0 & \text { for } r_{a b} \geq r_{c}\end{cases}
$$

The variable $\lambda_{a b}$, shorthand for $\lambda_{a b}(t)$, is a dimensionless dynamic variable representing the degree of intermixing of the polymers $a$ and $b$, which evolves over time based on the following first order stochastic differential equation:

$$
\mathrm{d} \lambda_{a b}=-\left(\lambda_{a b}-\lambda_{a b}^{e q}\right) \frac{\mathrm{d} t}{\tau}+\mathrm{d} \mathbf{W}_{a b}^{\lambda}
$$

where $\tau$ is the relaxation time and $\mathbf{W}_{a b}^{\lambda}$ is a Wiener process with time-uncorrelated increments satisfying:

$$
\left\langle\mathrm{d} \mathbf{W}_{a b}^{\lambda} \mathrm{d} \mathbf{W}_{a b}^{\lambda}\right\rangle=\left(\frac{2 k_{B} T}{\alpha}\right)\left(\frac{\mathrm{d} t}{\tau}\right) \mathbf{I} .
$$




\subsubsection{The FENE Potential}

The FENE potential $\Phi_{f}$ for the deformation of the finitely extensible nonlinear elastic polymer springs is given by:

$$
\Phi_{f}\left(r_{i}\right)=-\frac{1}{2} k r_{0}^{2} \sum_{i=1}^{N} \ln \left[1-\left(\frac{r_{i}}{r_{0}}\right)^{2}\right]
$$

where $r_{i}$ is the separation between the two lobes of the polymer dumbell $i, k$ is the spring constant, and $r_{0}$ is the maximum allowed deformation of the spring.

\subsubsection{The equation of state}

For the fluid, we use the commonly used pseudo-incompressible equation of state given by:[26, 27]

$$
P_{i}=P_{0}\left[\left(\frac{n_{i}^{f}}{\bar{n}^{f}}\right)^{7}-1\right]
$$

where $P_{0}$ is chosen such that the velocity of sound in the simulation is sufficiently large in order that the density fluctuations are sufficiently small, which results in a fluid that resembles an incompressible fluid.

\subsubsection{Definition of weight functions and system parameters}

For the polymer blobs, we have used a normalized weight function that has been used earlier in literature, where the polymer model RaPiD has been employed[18, 21, 22, 23]. It given by $w^{p}(r)$ :

$$
w^{p}(r)= \begin{cases}\frac{15}{2 \pi\left(r_{c}^{5}-\sigma^{5}\right)}\left(r_{c}-\sigma\right)\left(r_{c}+\sigma-2 r\right) & \text { for } r \leq \sigma \\ \frac{15}{2 \pi\left(r_{c}^{5}-\sigma^{5}\right)}\left(r-r_{c}\right)^{2} & \text { for } \sigma \leq r \leq r_{c} \\ 0 & \text { for } r \geq r_{c},\end{cases}
$$

where the cut-off $r_{c}$ is chosen as $2.5 \sigma$.

For the fluid blobs, we have chosen the normalized $M_{4}$ kernel commonly used in 
$\mathrm{SPH}[28]$ as the weight function. It is given by $w^{f}(r)$ :

$$
w^{f}(r)= \begin{cases}\frac{1}{4 \pi h^{3}}\left[\left(2-\frac{r}{h}\right)^{3}-4\left(1-\frac{r}{h}\right)^{3}\right] & \text { for } r \leq h \\ \frac{1}{4 \pi h^{3}}\left(2-\frac{r}{h}\right)^{3} & \text { for } h \leq r \leq 2 h \\ 0 & \text { for } r \geq 2 h,\end{cases}
$$

where $h$ is what is commonly referred to as the support of the weight function, which we have chosen as $h=2 \sigma$ for our simulations such that the cutoff radius $R_{c}$ for the fluid blobs is larger than the cut-off radius $r_{c}$ chosen for the polymer blobs. This is done intentionally because the weight function $w^{f}(r)$ must be able to accurately estimate the second order derivatives occurring in the equation of motion for the fluid blobs. Following the same logic, it immediately follows that for the polymer-fluid interactions, as we do not need to calculate any gradients, we can use the weight function $w^{p}(r)$ with a smaller cut-off $r_{c}$ instead of $w^{f}(r)$ with a larger cut-off $R_{c}$ for computational efficiency. Moreover, we must emphasize that we have chosen the resolution for the fluid $\bar{n}^{f}$ such that there are on average 8 particles within a sphere of radius $h$ so that there are about 64 neighbouring fluid blobs on average, which is sufficiently higher than the minimum required number for this weight function for 3D SPH simulations.[29] The values of the system parameters have been summarized in table 5.1.

It is pertinent to note that the relative resolution between the polymer and the fluid, lets call it $R=\sigma / h$, which we have chosen as $1 / 2$ for the work presented in this chapter, is actually a very important parameter. In principle, one should choose a high enough value for the relative resolution $R$ in order to accurately mimic the draining of the solvent through the polymers. Nevertheless, in practice, if one chooses a very large value for $R$, a direct consequence would be that the fluid blobs have a really small mass and hence a much smaller time-step would be required, which would drastically affect the efficiency of the method. Furthermore, in case of studying flow through a particular geometry, naturally a much larger number of fluid blobs would be required if the same length scale of the geometry is to be studied with smaller blobs. Thus, a very high resolution leads to computational problems. On the other hand, if the value is chosen to be too small, then there would be a large number of 
polymers per fluid blob. This leads to a conceptual problem because then one would have to capture the fluid flow field gradient within each fluid blob and transmit it to the polymers within the blob, which will need a modification of the equation of motion for the polymers adding an extra term accounting for the gradient of the fluid flow field within the fluid blob. Thus, we have chosen the sizes of the polymer blobs not very different from that of the fluid blobs.

The physical properties of the fluid, i.e. the density and viscosity, have been chosen to be consistent with those of water. The value of $P_{0}$ has been chosen high enough to ensure small density fluctuations and a large enough velocity of sound $c_{s}$ in the simulation, which can be calculated as:

$$
c_{s}=\sqrt{\frac{\partial P}{\partial \rho}}=\sqrt{\frac{7 P_{0}}{\rho}} .
$$

We have ensured that the velocities that we encounter in the simulations performed in this study are much smaller than this velocity of sound in our simulation. For the flow through a cylindrical porous medium in the diagonal direction, a ten times higher value of $P_{0}$ than mentioned in table 5.1 has been selected to ensure that the fluid does not break apart downstream of the cylindrical beam, which was found to occur at lower $P_{0}$ values. 
Table 5.1: Summary of system parameters

\begin{tabular}{llll}
\hline \hline System parameter & Symbol & Value & Unit \\
\hline Solute length scale & $\sigma$ & 5.0 & $\mu \mathrm{m}$ \\
Friction coefficient & $\xi$ & $1.0 \times 10^{-7}$ & $\mathrm{~kg} / \mathrm{s}$ \\
Number of Kuhn segments & $\mathrm{p}$ & 300,000 & - \\
Concentration of polymers & $C$ & 2.5 & $\mathrm{C}^{*}$ \\
Maximum number density of polymers & $n_{\text {max }}^{p}$ & $1.0 \times 10^{4}$ & $\mathrm{C}^{*}$ \\
Flory Huggins interaction parameter & $\chi$ & 0.5 & - \\
Strength of polymer interactions & $\alpha$ & 500 & $k_{B} T$ \\
Relaxation time & $\tau$ & 1.0 & $\mathrm{~s}$ \\
Spring constant & $k$ & 50 & $k_{B} T / \sigma^{2}$ \\
\hline Solvent length scale & $h$ & 10.0 & $\mu \mathrm{m}$ \\
Resolution of fluid & $\bar{n}^{f}$ & 8 & $\mathrm{particles} / \mathrm{h}^{3}$ \\
Density of fluid & $\rho$ & 1000 & $\mathrm{~kg} / \mathrm{m}^{3}$ \\
Viscosity of fluid & $\eta$ & 1.0 & $\mathrm{mPa} . \mathrm{s}$ \\
Pressure coefficient & $P_{0}$ & $0.13 \mathrm{~Pa}$ & \\
\hline Time step & $\mathrm{d} t$ & 10.0 & $\mu \mathrm{s}$ \\
Temperature & $T$ & 300 & $\mathrm{~K}$ \\
\hline \hline
\end{tabular}

\subsubsection{Interaction of the fluid with the solid}

For the correct solid-fluid interaction, there are two important conditions that need to be met - no penetration of fluid into the solid and a no-slip boundary condition at the solid-fluid interface. For this purpose, we explicitly embed artificial particles uniformly distributed in the solid regions with the same density as the real fluid blobs. These artificial particles interact with the real fluid blobs in a couple of ways. Firstly, they contribute to the density of the fluid blobs in their vicinity and consequently the pressure as well. Furthermore, the density and pressure is also calculated for the artificial particles in the vicinity of the real fluid blobs. This ensures that the pressure inside the solid is sufficient to prevent the fluid from entering the solid. Secondly, for ensuring the no-slip boundary condition at the surface of the solid, we apply the Morris boundary conditions,[30] which is applicable for plane as well as curved 
boundaries. We also use these artificial particles to calculate densities in the FloryHuggins potential for the polymers, albeit with a higher weight. The weight assigned to artificial particles is higher than regular polymer blobs by a ratio of the density of the polymers to the density of the artificial particles. This is to compensate for the difference in the density of these artificial particles and the polymers. This ensures that polymers do not artificially accumulate near the solid interface owing to lower concentration in a hypothetical sphere drawn around the center of these polymer blobs at the interface. Moreover, if still any polymer blob enters the solid due to a random displacement, we explicitly forbid that move, thereby preventing the polymer blob from entering the solid due to a random displacement.

\subsection{Results and Discussion}

\subsubsection{Flow through cylindrical porous media}

In this sub-section, we present results of flow of our model polymer solution through cylindrical porous media. By cylindrical porous media, we mean that our model porous media consist of solid cylindrical beams with axes along the $\mathrm{z}$ direction arranged on a square grid on the $\mathrm{x}-\mathrm{y}$ plane along the cartesian axes. The radius of each of the cylindrical beams is $40 \sigma$ i.e. 200 microns and the perpendicular distance between the axes of the cylinders in $\mathrm{x}$ as well as y direction is $90 \sigma$, i.e. 450 microns. This leads to a porous medium with a porosity of 0.38 . In all the simulations that we have presented in this chapter, we have maintained the same porosity. The simulation runs for the flow of polymer solutions are started with a steady state profile of the fluid and polymers are added randomly in a uniform manner throughout the box except in the solid region and then the run is continued till steady state is achieved.

\section{Pressure drop in the positive $\mathrm{x}$ direction}

In this subsection, we present results of flow through a cylindrical porous medium driven by an applied pressure drop in the $\mathrm{x}$ direction which is effected in the simulation through a body force which produces an acceleration of $0.1 \mathrm{~m} / \mathrm{s}^{2}$. In fig.5.1, we 
show the positions of polymers in our simulation at different times.

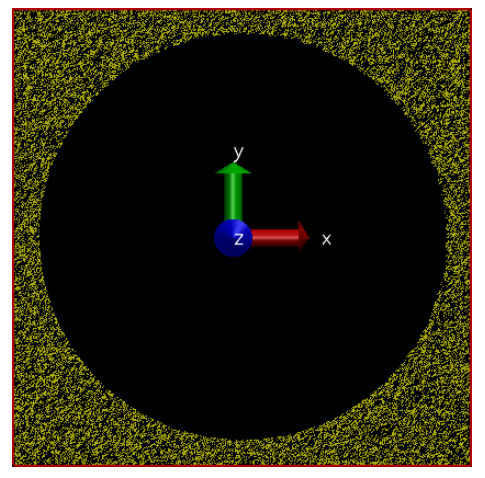

(a) $t=0 \mathrm{~s}$

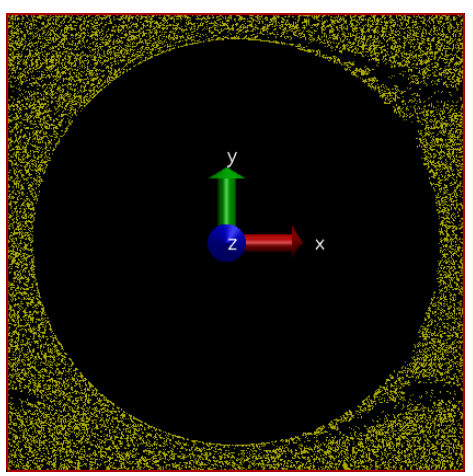

(c) $t=50 \mathrm{~s}$

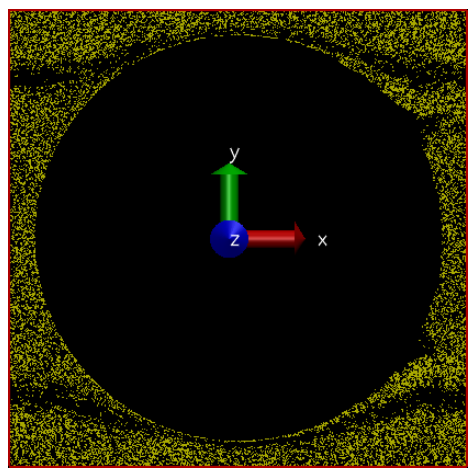

(e) $t=150 \mathrm{~s}$

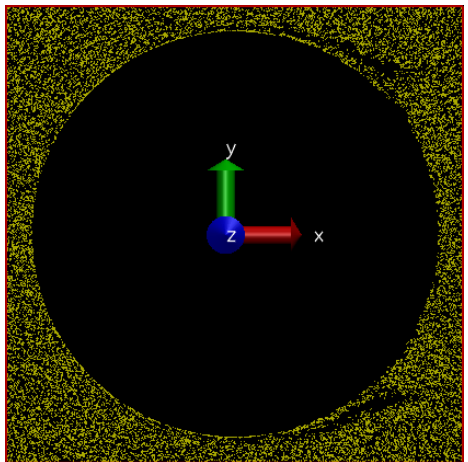

(b) $t=25 \mathrm{~s}$

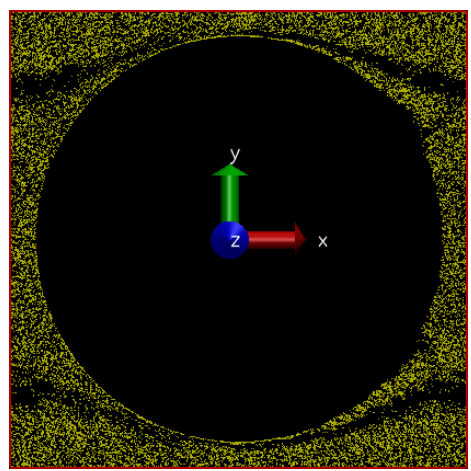

(d) $t=100 \mathrm{~s}$

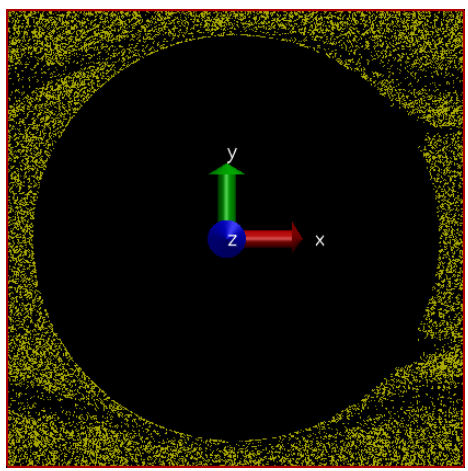

(f) $t=200 \mathrm{~s}$

Figure 5.1: Snapshots of simulation (in beam-view) at different times showing development of polymer positions over time. 
In this figure, we have shown the simulation box in what we call the 'beam-view', where the solid region is present at the center of the simulation box and the fluid flows around it. We have also shown the directions of the axes to clarify at the outset what we mean when we refer to $\mathrm{x}, \mathrm{y}$ and $\mathrm{z}$ directions. As can be seen in fig.5.1(a), the polymers are initially uniformly and randomly distributed throughout the box except the solid region which is the cylinder in the center of the box seen as a circle in this 2-D view. As time progresses, the polymers start forming a pattern which evolves over time and finally results in a more-or-less steady-state pattern at $200 \mathrm{~s}$ as shown in fig.5.1(f). It is interesting to note that the polymer distribution downstream of the beam is different from that upstream, although this effect is much more significant during early development, say at $25 \mathrm{~s}$ to 50 s, than later at steady state.

Since the simulation box is periodic in nature, we can also alternatively represent the results shown above in a different representation which we call the 'porous-mediaview' as shown in fig.5.2. In this view, the pore appears centrally and the solid region is at the corners. Note that we no longer show the axes in this figure and hereafter in all such figures as the notation has been already clarified in fig.5.1. Furthermore, we shall now present all the results in this porous-media-view as it provides a better visual impression of the porous media to the reader.

In fig.5.3, we have shown a comparison of the steady state velocity profiles of the flow of polymer solution through the porous medium with the flow of the solvent through the same medium for the same pressure drop. On the left hand side panels of such figures, we show the polymer solution flow profile and on the right hand side panels, we show the solvent flow profile. In fact, the polymer simulations are initiated with the fluid steady state profiles shown in the right panels. Note that the velocities in the legends of all such velocity-heatmap figures in this chapter are shown in internal units of $\sigma / \mathrm{s}$, i.e. the velocities in S.I. units [m/s] can be obtained by multiplying the velocities in internal units with $5 \times 10^{-6}$, as is shown in the captions. 


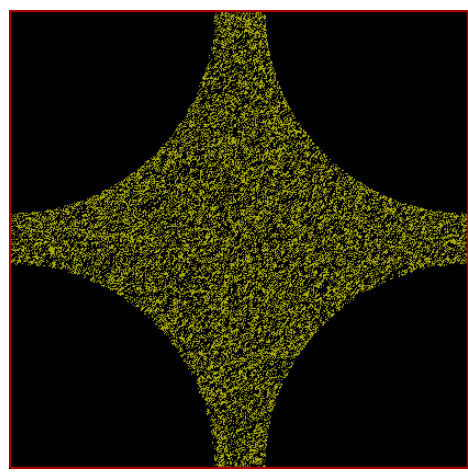

(a) $t=0 \mathrm{~s}$

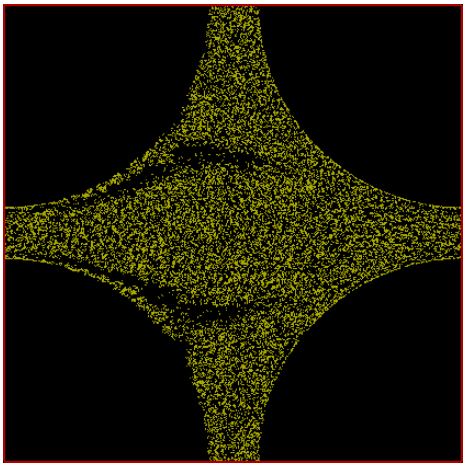

(c) $t=50 \mathrm{~s}$

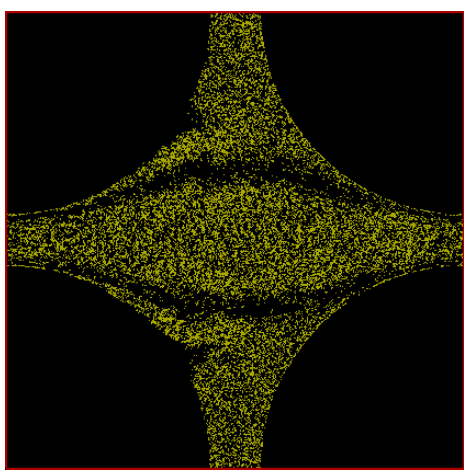

(e) $t=150 \mathrm{~s}$

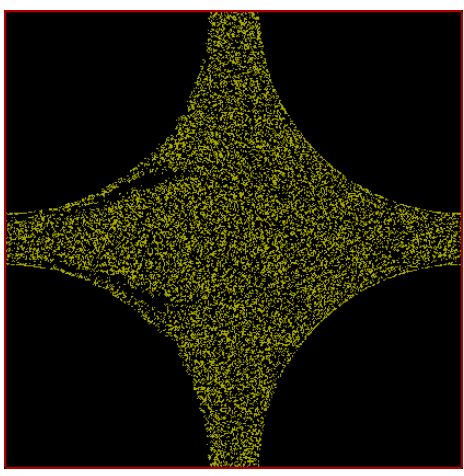

(b) $t=25 \mathrm{~s}$

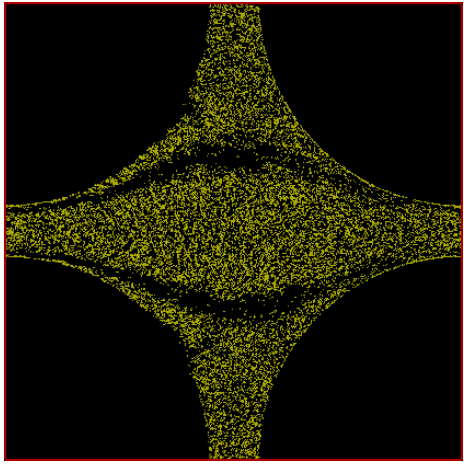

(d) $t=100 \mathrm{~s}$

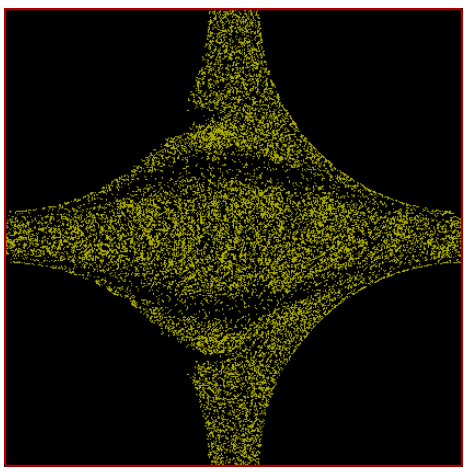

(f) $t=200 \mathrm{~s}$

Figure 5.2: Snapshots of simulation (in porous-media-view) at different times showing development of polymer positions over time. 
For a better understanding, we have shown not just the overall velocity but also its 2 components i.e. $v_{x}$ and $v_{y}$ in fig.5.3(a) and fig.5.3(c) respectively for the polymer solution flow and similarly in fig.5.3(b) and fig.5.3(d) for the solvent flow. Furthermore, in fig.5.3(e) and fig.5.3(f), we have shown the velocity vectors superimposed on the velocity-heatmap for the polymer solution flow and solvent flow respectively. From these two sub-figures, it can be seen that the maximum velocity $v$ in the polymer solution flow is almost 4.6 times lower than the same for the solvent flow through the same porous medium for the same pressure drop. This is due to increased viscosity of the solution due to the addition of polymers to the solvent. Furthermore, the viscosity of the polymer solution is not constant but rather shear-thinning in nature, which leads to the characteristic flattening of the velocity profile in the channels that is clearly visible for the polymer solution flow vis-a-vis the parabolic Poiseuille flow profile for the Newtonian solvent flow.

Furthermore, on comparison of fig.5.2 and fig.5.3(e), we can see that the polymer concentration is reduced in the zones separating the regions of strong primary flow with the regions of near stagnant flow. This shear induced cross-flow migration is similar to what we observed for the same model polymer solution under bulk shear flow in chapter 4 , where we observed that the polymers migrate to regions of low shear and move away from regions of high shear. This shows that the concentration of the polymers in the flow through the porous medium is not uniform but rather related to the flow through the particular geometry. This level of information is difficult to get with a continuum-level CFD simulation and it demonstrates the importance of particle-based simulations in linking the macroscopic flow to the microscopic molecular level interactions and positions of particles involved in the flow. Needless to say, a more detailed description of the molecules can provide even more information, but to go to a finer level of coarse-graining becomes very expensive if one has to simulate the flow of even several tens of thousands of polymers flowing through a model periodic porous medium like we show here. 

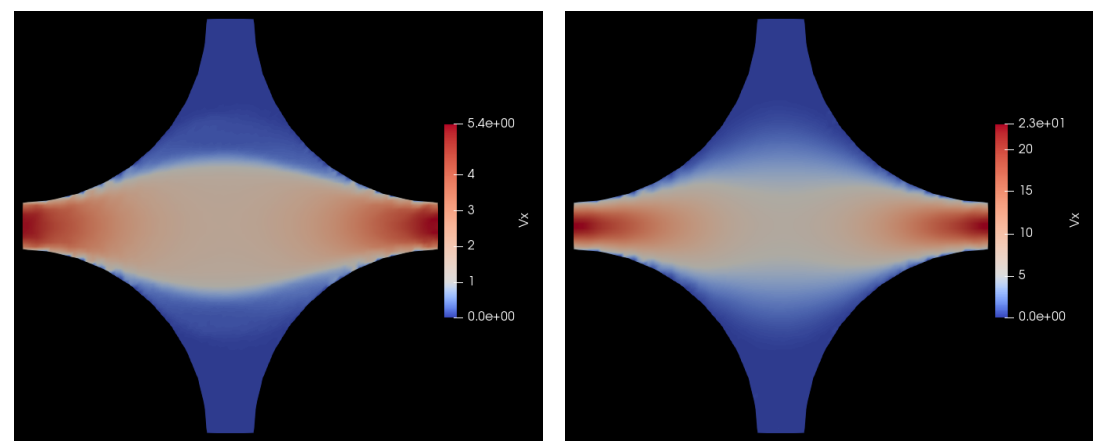

(a) $v_{x}$ for flow of polymer solution. Here, $v_{x}$ varies (b) $v_{x}$ for flow of solvent. Here, $v_{x}$ varies from 0 to from 0 to $5.4 \sigma / \mathrm{s}$, i.e. 0 to $27 \mu \mathrm{m} / \mathrm{s}$ $23 \sigma / \mathrm{s}$, i.e. 0 to $115 \mu \mathrm{m} / \mathrm{s}$
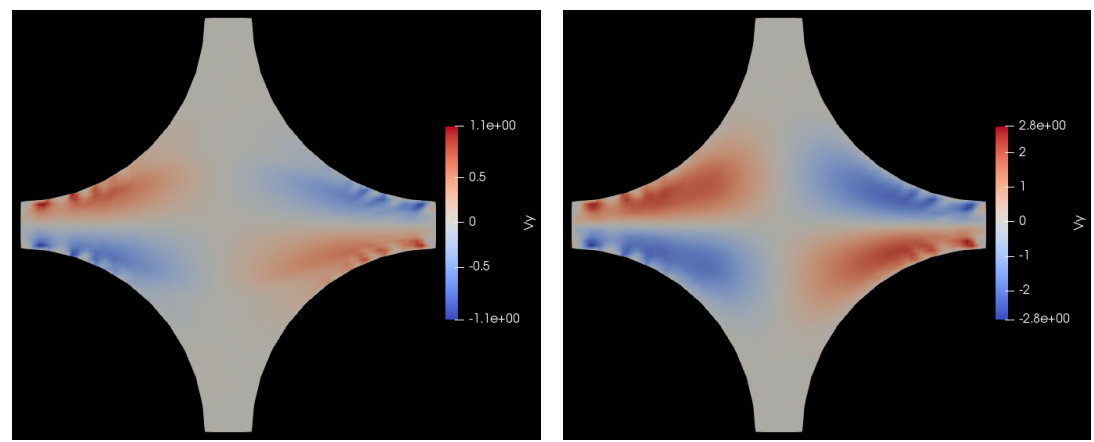

(c) $v_{y}$ for flow of polymer solution. Here, $v_{y}$ varies (d) $v_{y}$ for flow of solvent. Here, $v_{y}$ varies from -2.8 from -1.1 to $1.1 \sigma / \mathrm{s}$, i.e. -5.5 to $5.5 \mu \mathrm{m} / \mathrm{s}$ to $2.8 \sigma / \mathrm{s}$, i.e. -14 to $14 \mu \mathrm{m} / \mathrm{s}$
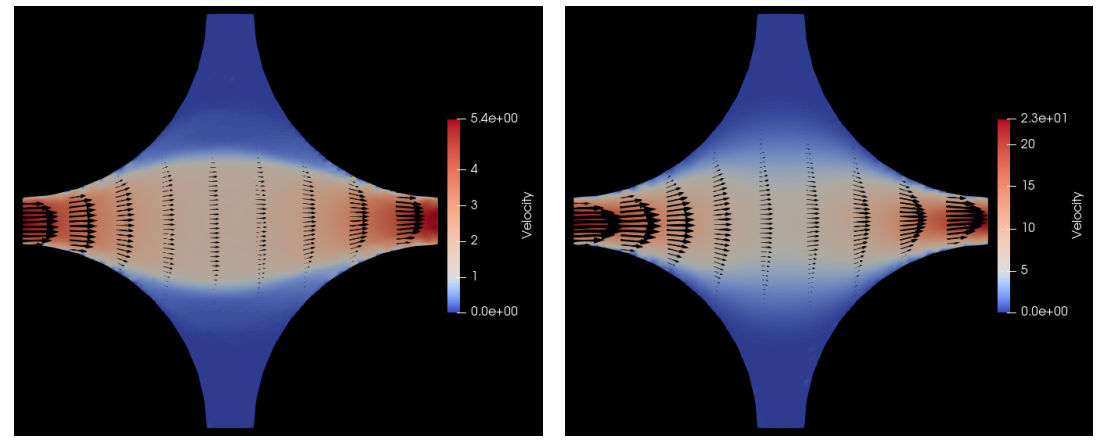

(e) $v$ for flow of polymer solution. Here, $v$ varies from (f) $v$ for flow of solvent. Here, $v$ varies from 0 to 23 0 to $5.4 \sigma / \mathrm{s}$, i.e. 0 to $27 \mu \mathrm{m} / \mathrm{s}$ $\sigma / \mathrm{s}$, i.e. 0 to $115 \mu \mathrm{m} / \mathrm{s}$

Figure 5.3: Comparison of velocity profiles for flow of polymer solution with the flow of solvent through a cylindrical porous medium with pressure applied in the positive $x$ direction 


\section{Pressure drop in the positive $\mathrm{x}$ and $\mathrm{y}$ directions}

In this subsection, we present results of flow through our model cylindrical porous medium driven by an applied pressure drop in the diagonal direction 45 degrees to the positive $\mathrm{x}$ and $\mathrm{y}$ directions. This is effected in the simulation through body forces along the positive $\mathrm{x}$ and $\mathrm{y}$ directions, which together produces an acceleration of 0.1 $\mathrm{m} / \mathrm{s}^{2}$ along the diagonal direction.

In fig.5.4, we show the positions of polymers at different times in our simulation box in porous-media-view. Here too, there is a difference between the downstream and upstream polymer distribution during the evolution of the flow, however it is less significant at the steady state.

In fig.5.5, we show the comparison between the velocity profiles for our model polymer solution $v s$ the Newtonian flow of the solvent. Besides the characteristic flattening of the shear thinning polymer solution flow through the channels as compared to the parabolic Poiseuille flow of the Newtonian solvent, there is another interesting aspect that can be observed by comparing the last two subfigures, i.e fig.5.5(e) and fig.5.5(f). There is not just a quantitative but also a qualitative difference in the velocity profile of the polymer solution vis-a-vis the fluid velocity profile.

Upon close observation of these two subfigures, one can see that the shape of the two stagnant regions in the bottom left and top right of the polymer solution flow shown in fig.5.5(e) is sharper (more pointed) as compared to the solvent flow in fig.5.5(f). This is clearly correlated with the polymer distribution that can be seen in fig.5.4(f). It can be seen that the polymers concentration is clearly reduced in the regions of high shear separating the primary flow from the stagnant regions and the resulting polymer concentration then affects the flow profile too because the resultant flow profile is qualitatively different from the solvent flow. This is a good illustration of the two-way coupling between the polymers and the background fluid, which is a key feature of our HCBD model. 


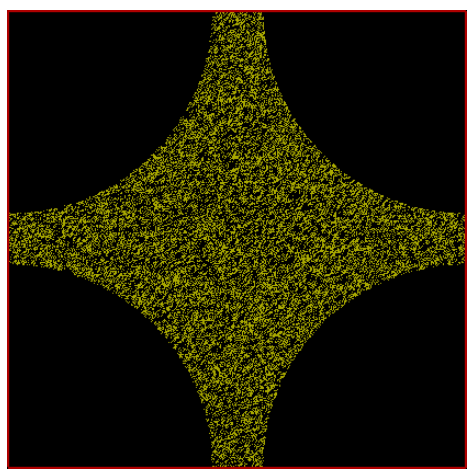

(a) $t=0 \mathrm{~s}$

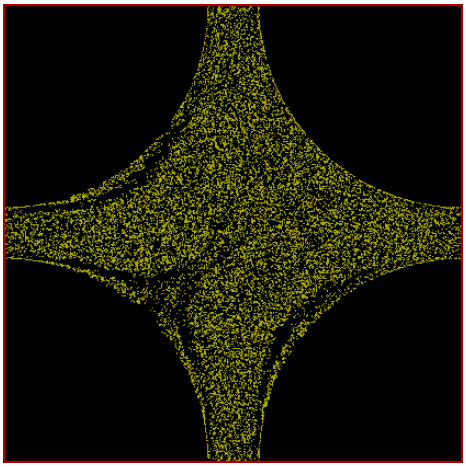

(c) $t=50 \mathrm{~s}$

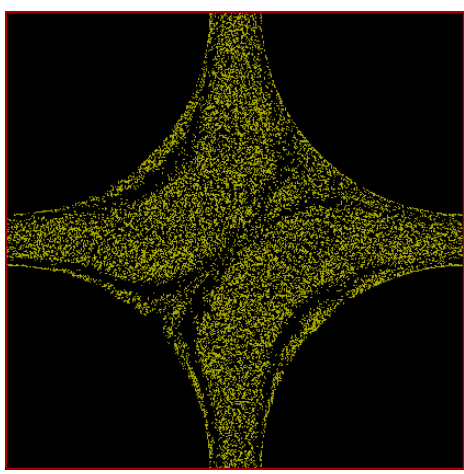

(e) $t=150 \mathrm{~s}$

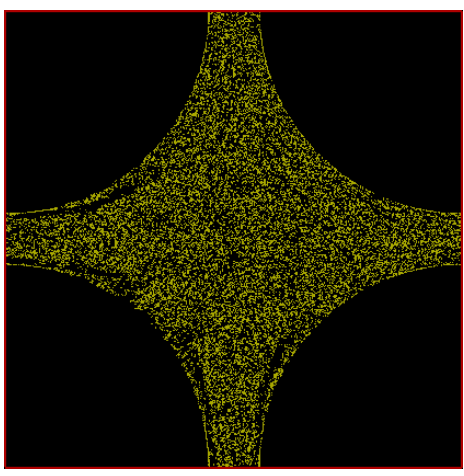

(b) $t=25 \mathrm{~s}$

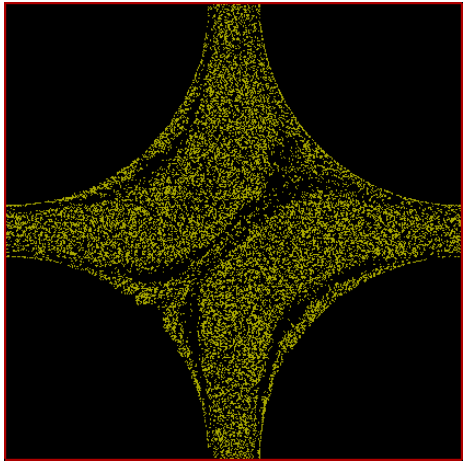

(d) $t=100 \mathrm{~s}$

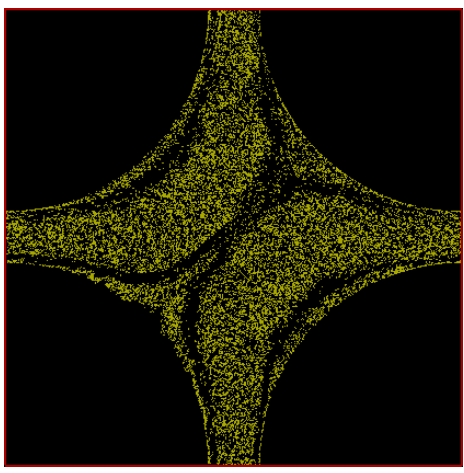

(f) $t=200 \mathrm{~s}$

Figure 5.4: Snapshots of simulation (in porous-media-view) at different times showing development of polymer positions over time. 

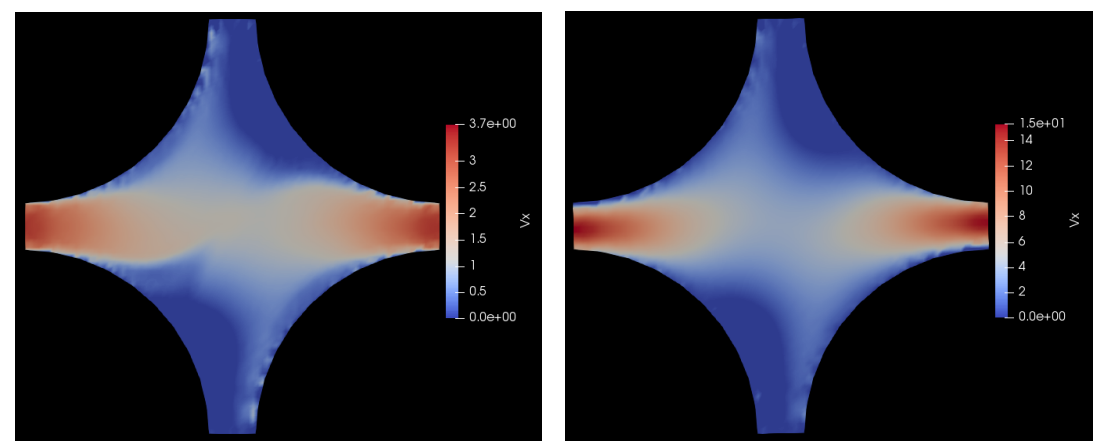

(a) $v_{x}$ for flow of polymer solution. Here, $v_{x}$ varies (b) $v_{x}$ for flow of solvent. Here, $v_{x}$ varies from 0 to from 0 to $3.7 \sigma / \mathrm{s}$, i.e. 0 to $18.5 \mu \mathrm{m} / \mathrm{s}$ $15 \sigma / \mathrm{s}$, i.e. 0 to $75 \mu \mathrm{m} / \mathrm{s}$
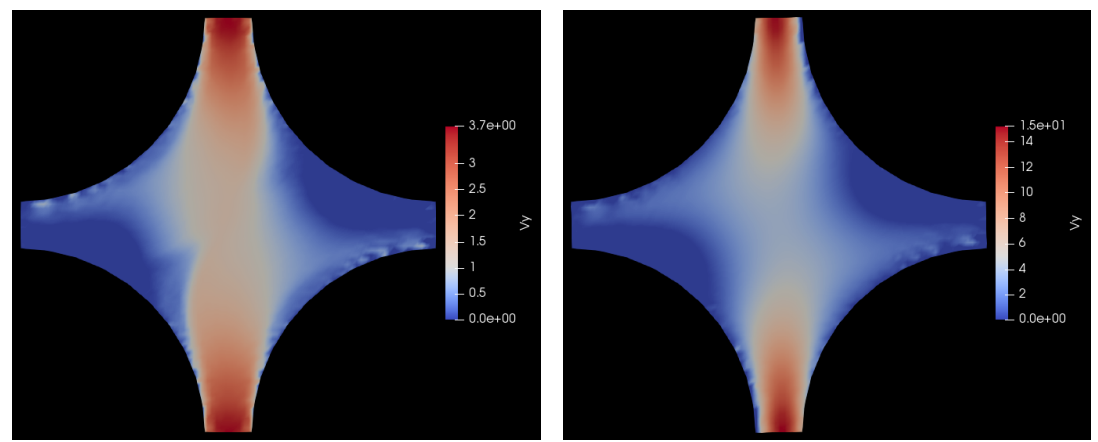

(c) $v_{y}$ for flow of polymer solution. Here, $v_{y}$ varies (d) $v_{y}$ for flow of solvent. Here, $v_{y}$ varies from 0 to from 0 to $3.7 \sigma / \mathrm{s}$, i.e. 0 to $18.5 \mu \mathrm{m} / \mathrm{s}$ $15 \sigma / \mathrm{s}$, i.e. 0 to $75 \mu \mathrm{m} / \mathrm{s}$
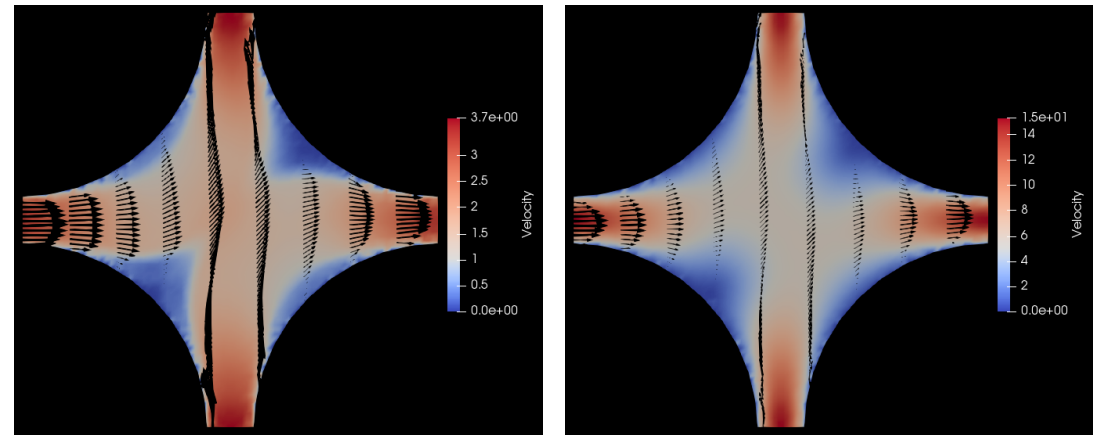

(e) $v$ for flow of polymer solution. Here, $v$ varies from (f) $v$ for flow of solvent. Here, $v$ varies from 0 to 15 0 to $3.7 \sigma / \mathrm{s}$, i.e. 0 to $18.5 \mu \mathrm{m} / \mathrm{s}$ $\sigma / \mathrm{s}$, i.e. 0 to $75 \mu \mathrm{m} / \mathrm{s}$

Figure 5.5: Comparison of velocity profiles for flow of polymer solution with the flow of solvent through a cylindrical porous medium with pressure applied in the positive $x$ and $y$ directions 


\subsubsection{Flow through cuboidal porous media}

In this sub-section, we present results of flow of our model polymer solution through cuboidal porous media. By cuboidal porous media, we mean that our model porous media consist of solid cuboidal beams with a square cross-section and the long axes oriented along the $\mathrm{z}$ direction arranged on a square grid on the $\mathrm{x}-\mathrm{y}$ plane along the cartesian axes. The length of the side of the square cross-section of each of these cuboidal beams is $71 \sigma$ i.e. 355 microns and the perpendicular distance between the axes of the beams in $\mathrm{x}$ as well as $\mathrm{y}$ direction is $90 \sigma$, i.e. 450 microns. This leads to a porous medium with a porosity of 0.38 , which is the same as the porosity that we had in case of the cylindrical beams. Again as in the case of cylindrical porous media simulations, the simulation runs for the flow of polymer solutions are started with a steady state profile of the fluid and polymers are added randomly in a uniform manner throughout the box except in the solid region and then the run is continued till steady state is achieved.

\section{Pressure drop in the positive $\mathbf{x}$ direction}

In this subsection, we present results of flow through a cuboidal porous medium driven by an applied pressure drop in the $\mathrm{x}$ direction which is effected in the simulation through a body force which produces an acceleration of $0.1 \mathrm{~m} / \mathrm{s}^{2}$. In fig.5.6, we show the positions of polymers at different times in our simulation box in porousmedia-view.

As you can see in 5.6(a), the polymers are initially uniformly and randomly distributed throughout the box except the solid region. As time progresses, the polymers start forming a pattern which evolves over time and finally results in a more-or-less steady-state pattern at $200 \mathrm{~s}$ as shown in fig.5.6(f). It is interesting to note that the polymer distribution downstream of the beam is different from that upstream during the development of the flow profile although this difference is much less conspicuous from that in the cylindrical porous media. In fig.5.7, we show a comparison of the steady state velocity profiles of the flow of polymer solution through the porous 


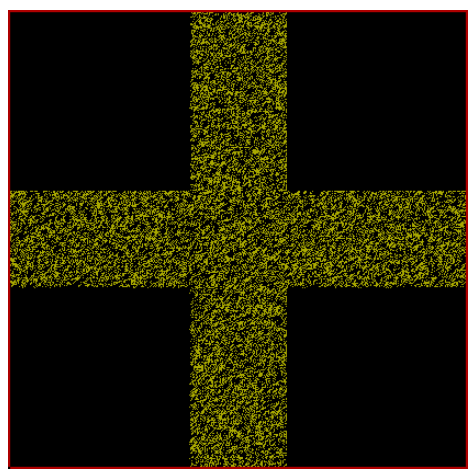

(a) $t=0 \mathrm{~s}$

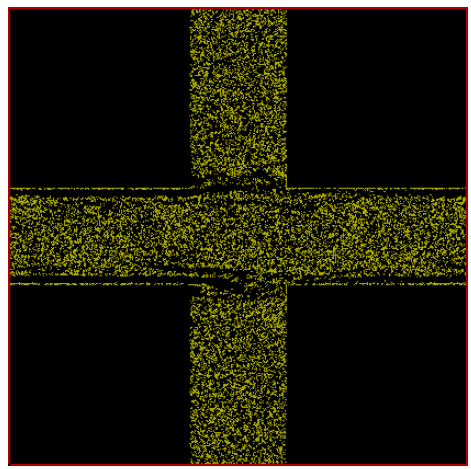

(c) $t=50 \mathrm{~s}$

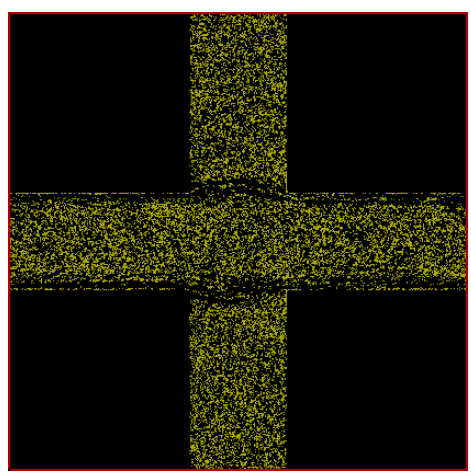

(e) $t=150 \mathrm{~s}$

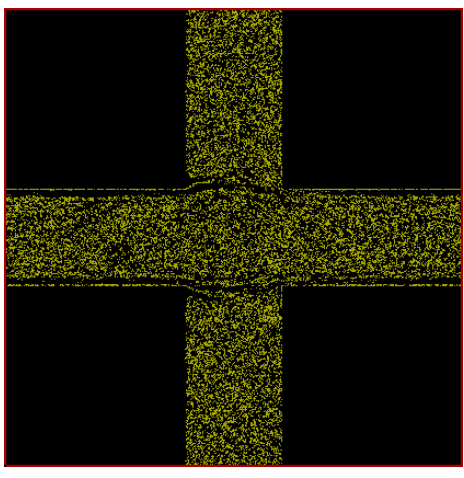

(b) $t=25 \mathrm{~s}$

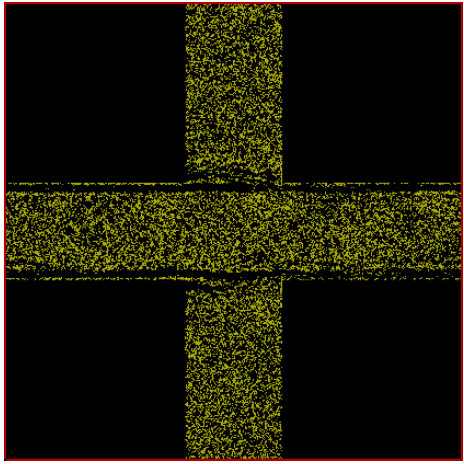

(d) $t=100 \mathrm{~s}$

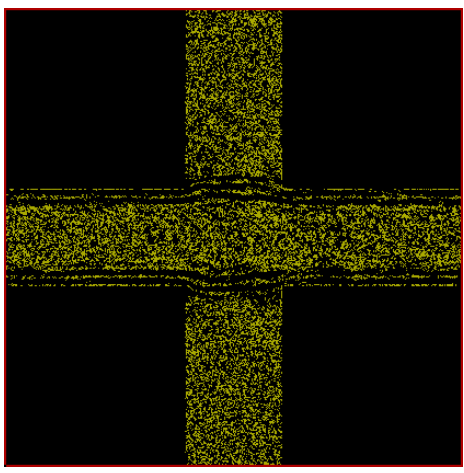

(f) $t=200 \mathrm{~s}$

Figure 5.6: Snapshots of simulation (in porous-media-view) at different times showing development of polymer positions over time. 

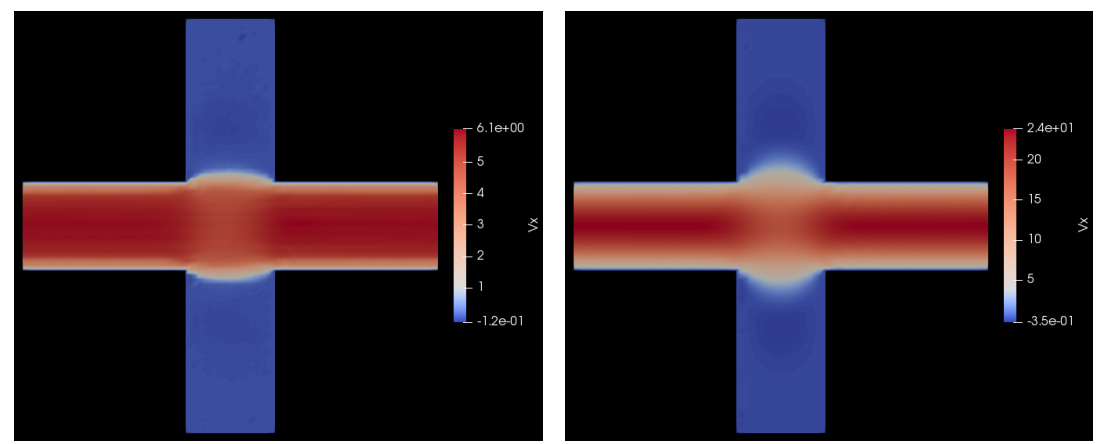

(a) $v_{x}$ for flow of polymer solution. Here, $v_{x}$ varies (b) $v_{x}$ for flow of solvent. Here, $v_{x}$ varies from -0.35 from -0.12 to $6.1 \sigma / \mathrm{s}$, i.e. -0.6 to $30.5 \mu \mathrm{m} / \mathrm{s}$ to $24 \sigma / \mathrm{s}$, i.e. -1.75 to $120 \mu \mathrm{m} / \mathrm{s}$
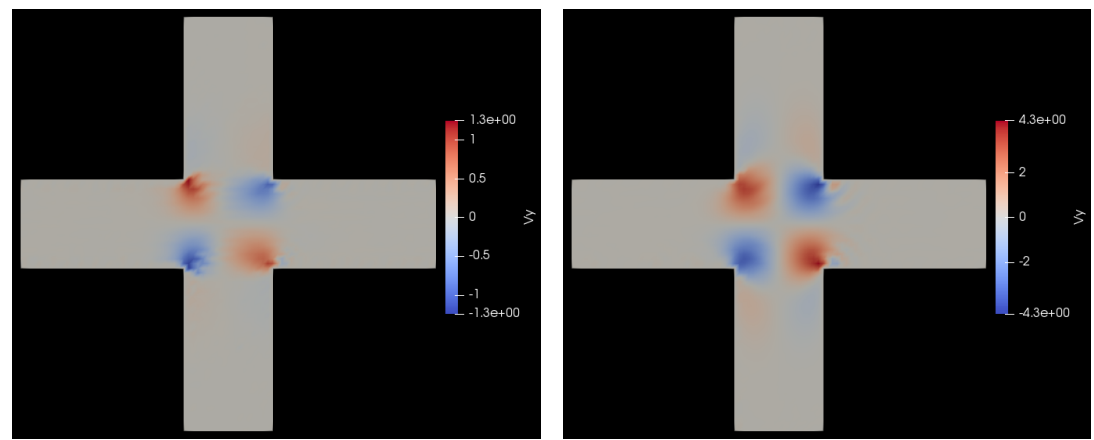

(c) $v_{y}$ for flow of polymer solution. Here, $v_{y}$ varies (d) $v_{y}$ for flow of solvent. Here, $v_{y}$ varies from -4.3 from -1.3 to $1.3 \sigma / \mathrm{s}$, i.e. -6.5 to $6.5 \mu \mathrm{m} / \mathrm{s}$ to $4.3 \sigma / \mathrm{s}$, i.e. -21.5 to $21.5 \mu \mathrm{m} / \mathrm{s}$
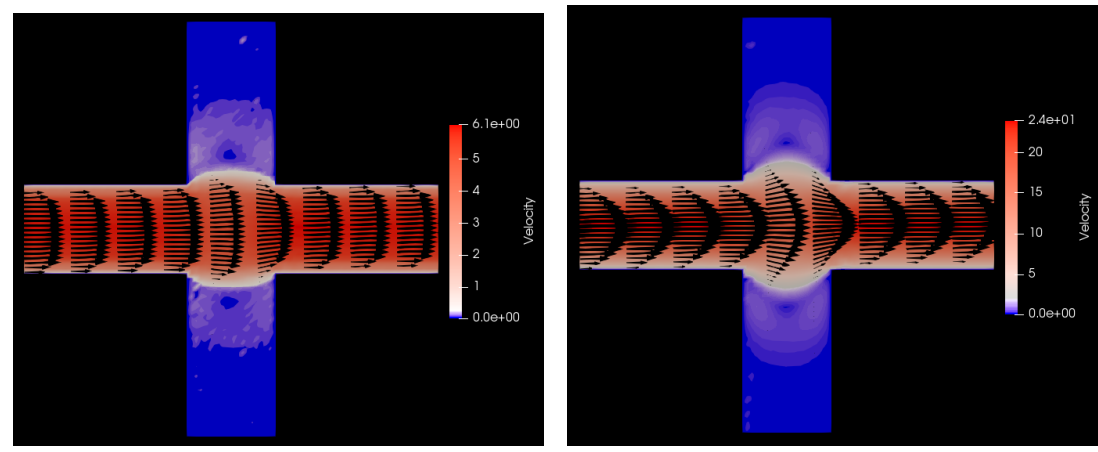

(e) $\mathbf{v}$ for flow of polymer solution. Here, $v$ varies from (f) $\mathbf{v}$ for flow of solvent. Here, $v$ varies from $\quad 0$ to 24 0 to $6.1 \sigma / \mathrm{s}$, i.e. 0 to $30.5 \mu \mathrm{m} / \mathrm{s}$ $\sigma / \mathrm{s}$, i.e. 0 to $120 \mu \mathrm{m} / \mathrm{s}$

Figure 5.7: Comparison of velocity profiles for flow of polymer solution with the flow of solvent through a cuboidal porous medium with pressure applied in the positive $x$ direction 
medium with the flow of the solvent through the same medium for the same pressure drop. On the left hand side panels of this figure, we show the polymer solution flow profile and on the right hand side panels, we show the solvent flow profile.

For this flow geometry, we see interesting secondary flows induced in the vertical channels above and below the horizontal flow. Because of the restrictive size of the channels, the primary flow in the $\mathrm{x}$ direction does not gradually dissipate in the $\mathrm{y}$ direction as in the case of the analogous cylindrical beam case, but rather leads to secondary flows. These secondary flows are essentially two counter-rotating vortices - one above and one below the primary horizontal flow, which can be seen in purple colour in the vertical columns in fig.5.7(e) and fig.5.7(f). Furthermore, in these two subfigures, the characteristic flattening of the velocity profile in the channels is clearly visible for the polymer solution flow vis-a-vis the parabolic Poiseuille flow profile for the Newtonian solvent flow.

\section{Pressure drop in the positive $\mathbf{x}$ and $\mathbf{y}$ directions}

In this subsection, we present results of flow through our model cuboidal porous medium driven by an applied pressure drop in the diagonal direction 45 degrees to the positive $\mathrm{x}$ and $\mathrm{y}$ directions. This is effected in the simulation through body forces along the positive $\mathrm{x}$ and $\mathrm{y}$ directions, which together produces an acceleration of 0.1 $\mathrm{m} / \mathrm{s}^{2}$ along the diagonal direction. In fig.5.8, we show the positions of polymers at different times in our simulation box in porous-media-view. There is a difference between the downstream and upstream polymer distribution during the development of the flow in this case as well but is less conspicuous than the corresponding cylindrical porous medium case.

In fig.5.9, we show the comparison between the velocity profiles for our model polymer solution $v s$ the Newtonian flow of the solvent. It can be observed from the last two subfigures of the above figure, i.e fig.5.9(e) and fig.5.9(f) that there is a characteristic flattening of the polymer solution flow through the channels as compared to the parabolic Poiseuille flow of the Newtonian solvent. Furthermore, there is also a qualitative difference between the velocity profile of the polymer solution 


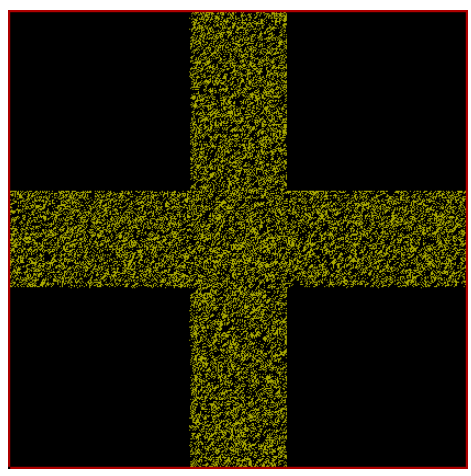

(a) $t=0 \mathrm{~s}$

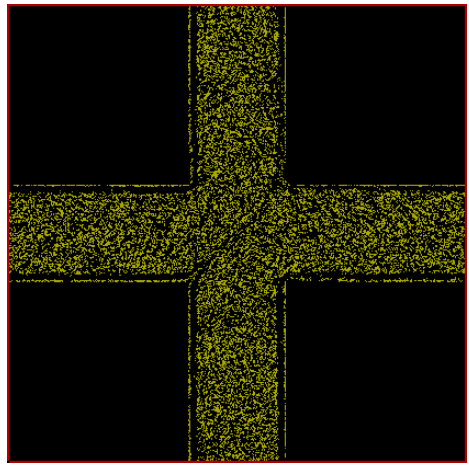

(c) $t=50 \mathrm{~s}$

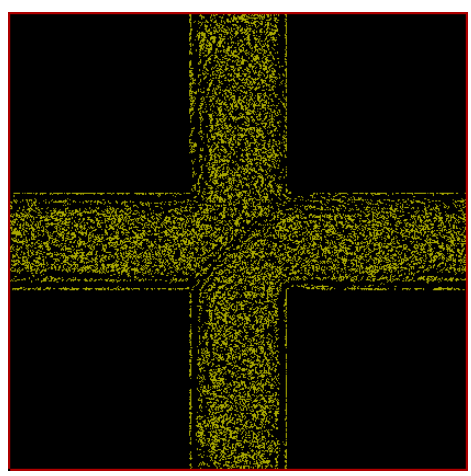

(e) $t=150 \mathrm{~s}$

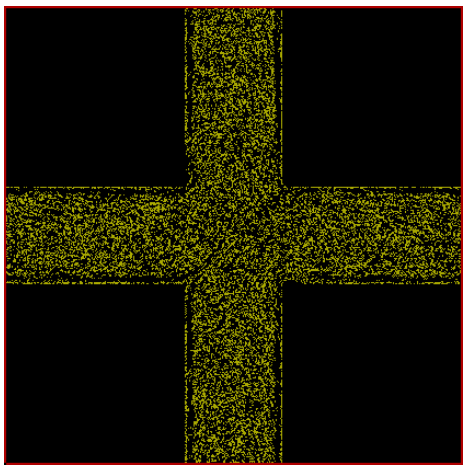

(b) $t=25 \mathrm{~s}$

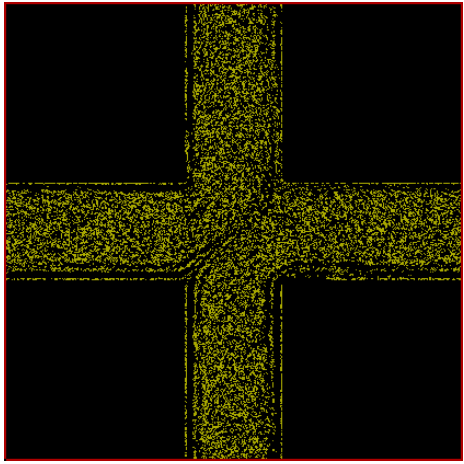

(d) $t=100 \mathrm{~s}$

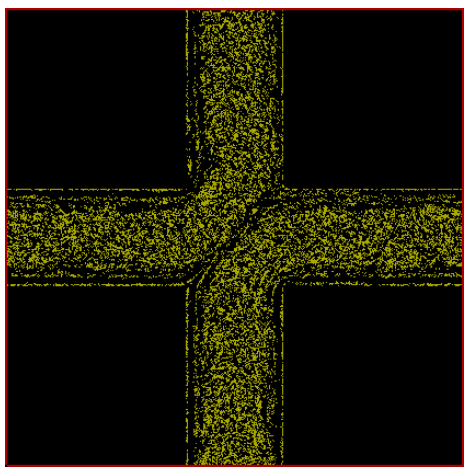

(f) $t=200 \mathrm{~s}$

Figure 5.8: Snapshots of simulation (in porous-media-view) at different times showing development of polymer positions over time. 

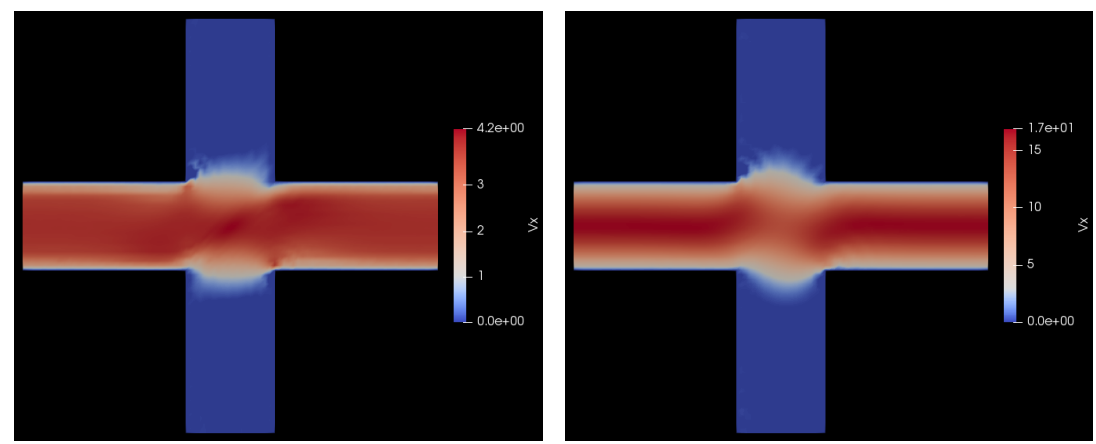

(a) $v_{x}$ for flow of polymer solution. Here, $v_{x}$ varies (b) $v_{x}$ for flow of solvent. Here, $v_{x}$ varies from 0 to from 0 to $4.2 \sigma / \mathrm{s}$, i.e. 0 to $21 \mu \mathrm{m} / \mathrm{s}$ $17 \sigma / \mathrm{s}$, i.e. 0 to $85 \mu \mathrm{m} / \mathrm{s}$
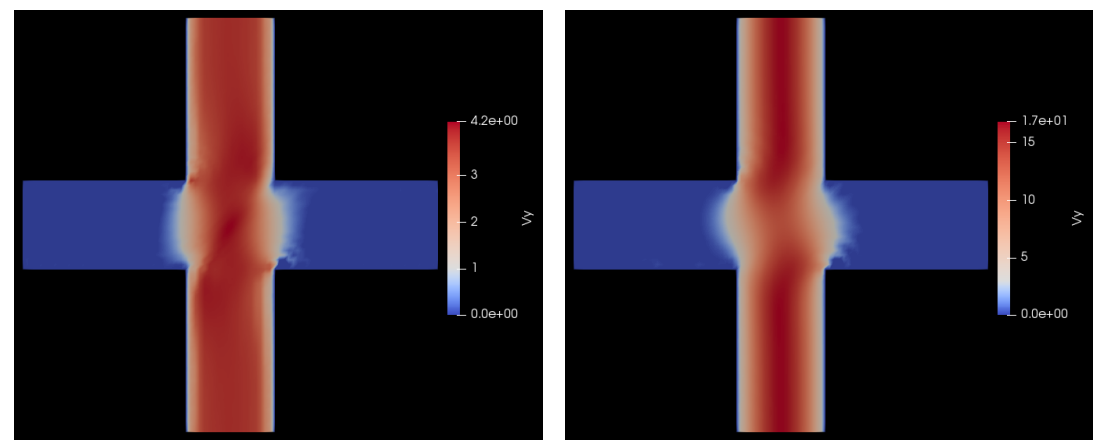

(c) $v_{y}$ for flow of polymer solution. Here, $v_{y}$ varies (d) $v_{y}$ for flow of solvent. Here, $v_{y}$ varies from 0 to from 0 to $4.2 \sigma / \mathrm{s}$, i.e. 0 to $21 \mu \mathrm{m} / \mathrm{s}$ $17 \sigma / \mathrm{s}$, i.e. 0 to $85 \mu \mathrm{m} / \mathrm{s}$
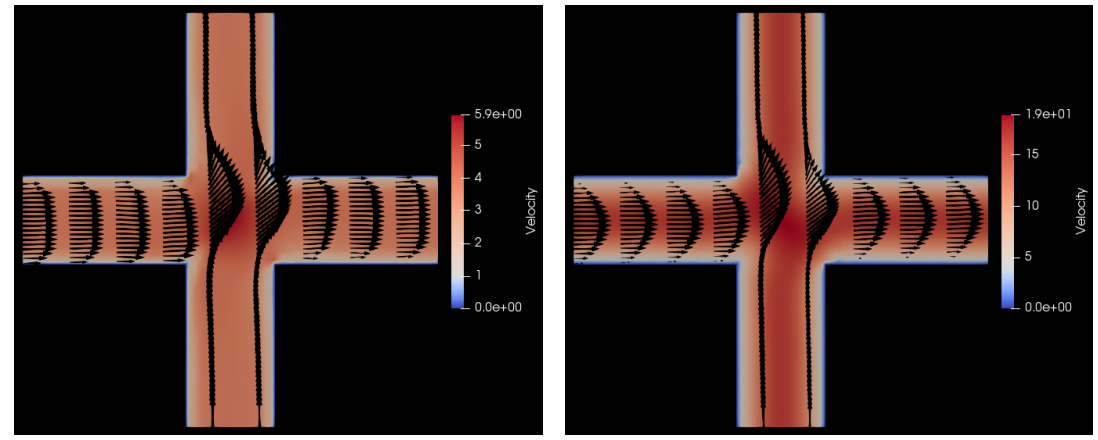

(e) $v$ for flow of polymer solution. Here, $v$ varies from (f) $v$ for flow of solvent Here, $v$ varies from 0 to 19 0 to $5.9 \sigma / \mathrm{s}$, i.e. 0 to $29.5 \mu \mathrm{m} / \mathrm{s}$ $\sigma / \mathrm{s}$, i.e. 0 to $95 \mu \mathrm{m} / \mathrm{s}$

Figure 5.9: Comparison of velocity profiles for flow of polymer solution with the flow of solvent through a cuboidal porous medium with pressure applied in the positive $x$ and $y$ directions 
and that of the fluid, which in this case is more evident from the comparison of the velocity components, i.e. comparison of the $v_{x}$-heatmaps in fig.5.9(a) with fig.5.9(b) and comparison of the $v_{y}$-heatmaps in fig.5.9(c) with fig.5.9(d) respectively. This can be correlated with the polymer concentration at steady state in fig.5.8(f). As the polymers at the intersection of the two primary flows get sheared, there results in a depletion in this zone due to the transient forces of the polymers, which then affects the fluid flow profile.

\subsection{Conclusion and scope for further research}

We have studied the flow of model polymer solution through two different types of model porous media - one made up of a periodic array of cylindrical beams with circular cross-section and another made up of a periodic array of cuboidal beams with a square cross-section. The axes of the beams are beams are perpendicular to a plane, on which they are arranged with a square pitch. The distance between the axes of the beams along the two cartesian directions of this plane is calculated so as to have a porosity of 0.38 in both cases. We have studied the flow through these two types of porous media at two extreme angles of approach by applying pressure drop in two extreme flow directions - one parallel to one of the cartesian directions of the plane on which the beams are arranged and another at 45 degrees to both the cartesian directions of the plane. Thus, we have studied two different flow configurations of flow through two different types of model porous media. We have compared our results of the polymer solution flow through these porous media with the Newtonian flow through the same media for the same applied pressure drop. We observe that there are significant quantitative and qualitative differences between the Newtonian flow of the solvent and the non-Newtonian flow of our model polymer solution through the same porous media for the same applied pressure drop.

The addition of the polymers to the solvent increases the viscosity of the solvent, which leads to a reduction in the velocity of the flow of the polymer solution through the porous media. Furthermore, this increased viscosity of the polymer solution is not 
constant but rather shear dependent. We studied the response of this model polymer solution to pure shear flow in chapter 4. In this chapter, we see how this leads to a flatter velocity profile of the shear-thinning polymer solution in the channels as compared to the parabolic Poiseuille flow profile of the Newtonian solvent.

We start our polymer solution simulation with the steady-state flow profile of the Newtonian solvent and add polymers uniformly to the simulation box in the pore region (the gaps left by the solid regions). The initial variation in the shear rates at different points in space in the Newtonian flow profile leads to cross-flow migration of polymers away from regions of high shear separating the regions of strong primary flow with near-stagnant regions. Gradually, a steady state pattern evolves for the polymer concentration which then in turn affects the flow and finally leads to a flow profile that is fundamentally different from the Newtonian flow profile. Thus, the two-way coupling of our HCBD model leads to the flow affecting the polymer concentration at different points in space and the resultant polymer microstructures in turn affecting the flow through the porous media. Thus, we have provided a proof of concept of how a technique that couples microscopic interactions between polymer molecules with the flow of the background solvent can be applied to study flow of a model polymer solution through porous media. In future work, we can perform a sensitivity analysis of the molecular parameters to further the understanding of how the molecular level interactions manifest themselves in the macroscopic flow of polymer solutions.

Furthermore, we have shown that we can perform flow simulations of polymer solutions not just with rounded obstacles like cylindrical beams but also with obstacles with sharp corners such as cuboidal beams. Thus, this method allows us to study more complex geometries that may exist in real life situations such as oil-reservoirs much better than continuum level simulations, albeit restricted to a smaller scale. We can further envisage that a hybrid model may be constructed where our technique could be used to feed information from the scale of pore throats to larger scale Computational Fluid Dynamics (CFD) simulations of oil reservoirs. In this initial study, we did not model the oil phase but rather focused on the single phase flow of polymer 
solution through the porous media, as that in itself is a complex subject. In future works, we plan to model the oil phase as well and simulate the full multiphase flow through porous media that is involved in the polymer flooding operations. In principle, it should not be very difficult to add another Newtonian oil phase to our nonNewtonian polymer solution simulation but it will still require tuning the interaction between the aqueous and the oil phase in order to produce the proper thermodynamic interaction between the two phases. 


\section{Bibliography}

[1] A. Thomas, N. Gaillard, and C. Favero, Oil \& Gas Science and TechnologyRevue dIFP Energies nouvelles, 67, 887 (2012).

[2] S. Thomas, Oil \& Gas Science and Technology-Revue de l'IFP, 63, 9 (2008).

[3] M. A. Nilsson, R. Kulkarni, L. Gerberich, R. Hammond, R. Singh, E. Baumhoff, and J. P. Rothstein, Journal of Non-Newtonian Fluid Mechanics, 202, 112 (2013).

[4] K. S. Sorbie, Polymer-Improved Oil Recovery (CRC Press Inc., Boca Raton, FL, 1991).

[5] The American Petroleum Institute gravity of a fluid with specific gravity SG is defined as: ${ }^{\circ} \mathrm{API}=\frac{141.5}{\mathrm{SG}}-131.5$. It essentially indicates how different the density of a petroleum liquid is compared to that of water. If ${ }^{\circ}$ API gravity of a fluid is greater than 10 , it is lighter than water and if less than 10 , it is heavier than water.

[6] R. Marshall and A. Metzner, Industrial \& Engineering Chemistry Fundamentals 6, 393 (1967).

[7] D. F. James and D. McLaren, Journal of Fluid Mechanics 70, 733 (1975).

[8] S. Rodriguez, C. Romero, M. Sargenti, A. Müller, A. Sáez, and J. Odell, Journal of Non-Newtonian Fluid Mechanics 49, 63 (1993).

[9] C. Chmielewski and K. Jayaraman, Journal of Non-Newtonian Fluid Mechanics 48, 285 (1993).

[10] K. K. Talwar and B. Khomami, Journal of Non-Newtonian Fluid Mechanics 57, 177 (1995).

[11] P. Sousa, F. Pinho, M. Oliveira, and M. Alves, Journal of Non-Newtonian Fluid mechanics 165, 652 (2010). 
[12] F. J. Galindo-Rosales, L. Campo-Deaño, F. Pinho, E. Van Bokhorst, P. Hamersma, M. S. Oliveira, and M. Alves, Microfluidics and nanofluidics 12, 485 (2012).

[13] A. W. Liu, D. E. Bornside, R. C. Armstrong, and R. A. Brown, Journal of Non-Newtonian Fluid Mechanics 77, 153 (1998).

[14] M. A. Hulsen, R. Fattal, and R. Kupferman, Journal of Non-Newtonian Fluid Mechanics 127, 27 (2005).

[15] D. Richter, G. Iaccarino, and E. S. Shaqfeh, Journal of Fluid Mechanics 651, 415 (2010).

[16] S. De, J. Kuipers, E. Peters, and J. Padding, Physical Review Fluids 2, 053303 (2017).

[17] S. De, J. Kuipers, E. Peters, and J. Padding, Journal of Non-Newtonian Fluid Mechanics 248, 50 (2017).

[18] V. R. Ahuja, J. van der Gucht, and W. J. Briels, The Journal of Chemical Physics 148, 034902 (2018).

[19] A. Lees and S. Edwards, Journal of Physics C: Solid State Physics 5, 1921 (1972).

[20] V. R. Ahuja, J. van der Gucht, and W. J. Briels, The Journal of Chemical Physics 145, 194903 (2016).

[21] I. S. Santos de Oliveira, A. van den Noort, J. T. Padding, W. K. den Otter, and W. J. Briels, The Journal of Chemical Physics 135, 104902 (2011).

[22] I. S. Santos de Oliveira, W. K. den Otter, and W. J. Briels, The Journal of Chemical Physics 137, 204908 (2012).

[23] I. S. Santos de Oliveira, B. W. Fitzgerald, W. K. den Otter, and W. J. Briels, The Journal of Chemical Physics 140, 104903 (2014). 
[24] A. Van den Noort, W. K. den Otter, and W. J. Briels, Europhysics Letters 80, 28003 (2007).

[25] W. J. Briels, Soft Matter 5, 4401 (2009).

[26] G. K. Batchelor, An Introduction to Fluid Dynamics (Cambridge university press, 2000).

[27] R. H. Cole, Underwater explosions (Dover Publications, 1965).

[28] J. J. Monaghan, Reports on Progress in Physics 68, 1703 (2005).

[29] A. P. Whitworth, A. Bhattal, J. Turner, and S. Watkins, Astronomy and Astrophysics 301, 929 (1995).

[30] J. P. Morris, P. J. Fox, and Y. Zhu, Journal of Computational Physics 136, 214 (1997). 


\section{Summary}

The flow of non-Newtonian fluids, polymer solutions in particular, has been a subject of research for several decades. Owing to the complexity of this subject, many fundamental questions are still open for discussion even today. Particularly, the flow of these complex fluids through complex geometries is a poorly understood subject, and over time the knowledge in this field has been growing with advances in both experimental as well as simulation techniques. In this thesis, we have described a novel simulation technique that we have developed for studying this problem. With this technique, we have provided a new methodology to study the bulk flow of polymer solutions as well as their flow through porous media.

In Chapter 2, we have presented a coarse-grain particle based simulation technique in which we couple the Brownian motion of the polymers with the flow of a 'background fluid' implicitly present at the positions of the centers-of-mass of the polymers. The polymers are represented by the positions of their centers-of-mass and interact with each other based on pair potentials. As our test system, we used a potential that is commonly used for modeling star polymer solutions. Furthermore, the polymers are also influenced by the flow of the background fluid, which is calculated by solving a modified version of the Navier-Stokes equation on a moving grid of nodes, which in this case are positioned at the centers-of-mass of the polymers. The modification to the Navier-Stokes equation is essentially the addition of an extra force term, which is actually the force felt by the polymers at that position due to the interaction with the other polymers. This method of transmitting the polymer force to the fluid, which then affects back the flow of the polymers is based on the Galilean invariant, first order Brownian dynamics algorithm developed by Padding and Briels [J. Chem. Phys.141, 244108 (2014)]. This makes the flow of the background fluid non-Newtonian and also couples it to the polymers. This coupling is straightforward as the fluid blobs are assumed to be implicitly present at the positions of the centersof-mass of the polymers. We have applied this method to study the flow of the model star polymer solution between two infinite solid plates. For this purpose, we applied appropriate boundary conditions at the solid-fluid interface using the method devel- 
oped by Morris et al. [J. Comp. Phys. 136, 214 (1997)].

Although the method described in Chapter 2 gives good results and is computationally very efficient, yet there a couple of limitations of this method, viz. one is not free to choose the resolution of the background fluid as it is by definition the concentration of the polymers, and one is not free to choose the equation of the state for the fluid either if the method is used as is. Therefore, we envisaged that the model can be improved by using two different types of particles for the polymers and the fluid. However, the coupling between the two would not be obvious like the previous method because now the fluid blobs are not present at the position of the centersof-mass of the polymers anymore. So we developed a novel approach which we call Hydrodynamically Coupled Brownian Dynamics (HCBD), in which we couple the polymer blobs and the fluid blobs (which are now independent entities), using a special interaction term that we constructed in such a way that the momentum is conserved locally. We describe the development of this technique along with simple applications in Chapter 3, where we chose the Flory-Huggins potential to describe the interactions between the polymers and also introduced memory into the system using the Responsive Particle Dynamics (RaPiD) method. Using this model, we studied the rheology of model linear polymer solutions for two different concentrations by simulating their responses to a homogeneous shear using the Lees-Edwards method and to a varying shear environment using the Reverse Poiseuille Flow technique. We observed that the polymers are coupled very well with the fluid, which is evident from the absence of a lag between their flows. Furthermore, we observed the characteristic shear-thinning and cross-flow migration phenomenon, that have been observed experimentally.

In Chapter 4, we used our novel HCBD technique to couple the background fluid with a less coarse and hence more realistic polymer model viz. RaPiD with Finitely Extensible Non-Linear Elastic (FENE) dumbbells. In this polymer model, the polymers are represented as a finitely extensible non-linear elastic dumbbell instead of a point particle as in the case of RaPiD. This brings, in addition to the external, quasi elastic memory term of RaPiD, intra-molecular elasticity in the system and allows us 
to study elastic instabilities exhibited by polymer solutions. We simulated the flow of this model polymer solution for two different concentrations in a homogeneous shear field. For the highest concentration, we observe the well known shear banding phenomenon and study its time evolution. Moreover, we have found that at certain shear rates the resulting bands themselves become unstable and spontaneously form microstructures such as 'shear rolls' and 'streaks'.

In Chapter 5, we studied the flow of our model polymer solution through a periodic array of cylindrical and cuboidal structures in two extreme flow directions. The complex geometries were constructed in such a way that they mimic various configurations of porous media. The porosity was maintained the same for the two different types of porous media and also the same pressure drop was applied across the media. Furthermore, two different, extreme current directions have been chosen in both cases. We have observed the differences in the emerging flow patterns and polymer concentrations in the two different media. We see interesting correlations between the polymer concentrations and the flow profiles due to cross-flow migration of the polymers. This highlights an advantage of our coarse-grain particle-based model in contrast to conventional CFD methods, as our method allows polymers to distribute heterogeneously over the accessible volume.

Thus, we have developed a novel computational technique (HCBD), which can be used to couple any Brownian Dynamics based polymer model with a background fluid model based on Smoothed Particle Hydrodynamics. We have shown that with our model we can study the flow of polymer solutions through porous media, which is one of the main applications of this work. An important aspect of our method is that it is not limited to studying flow of polymer solutions but can be easily extended to study the flow of viscoelastic surfactants or multiphase flow through porous media to understand how polymer solutions displace oil from an oil reservoir. This can greatly help improve the understanding of polymer flooding operations in Enhanced Oil Recovery. The additional oil recovered from the reservoirs can then be used for making petrochemical products or fuels to provide additional energy to the global market whose energy consumption is ever-increasing. Our work can also be used 
to improve the understanding of other operations in the chemical industry, such as polymer extrusion and 'food processing'. It is thus a new tool which can be used to further the scientific understanding for various phenomena of interest related to a wide range of non-Newtonian fluids using computer simulations. 


\section{Samenvatting}

Stroming van niet-Newtonse vloeistoffen, in het bijzonder die van polymeer oplossingen, is sinds jaren een onderwerp van intensief onderzoek. Vanwege de complexiteit van dit onderwerp zijn nog veel fundamentele vragen onbeantwoord. Vooral stroming van dergelijke vloeistoffen door complexe geometrieën wordt nog slecht begrepen, hoewel kennis van dit onderwerp gestaag groeit als gevolg van toenemende mogelijkheden in zowel experimentele als simulatie technieken. Dit proefschrift beschrijft een nieuw simulatie techniek, die is ontwikkeld om dit probleem in zijn volle complexiteit te bestuderen. Deze techniek kan zowel gebruikt worden om bulkstroming van polymeer oplossingen te bestuderen, als ook hun stroming door poreuze media.

Hoofdstuk 2 beschrijft een simulatie techniek met grofkorrelige deeltjes waarin de Brownse beweging van de polymeren is gekoppeld met stroming van een achtergrond vloeistof die impliciet aanwezig is op de plaats van de massamiddelpunten van de polymeren. Ieder polymeer wordt gemodelleerd als één deeltje, gerepresenteerd door de positie van zijn massamiddelpunt. De polymeren interacteren met elkaar door middel van paarwisselwerkingen. Als testsysteem is een model gebruikt dat vaak wordt gehanteerd voor het modelleren van sterpolymeer oplossingen en waarin de deeltjes interacteren door middel van de potentiaal van de gemiddelde kracht. Verder worden in dit hoofdstuk de polymeren ook meegesleurd door de stroming van een achtergrond vloeistof, die wordt beschreven door middel van een gemodificeerde Navier-Stokes vergelijking op een bewegend rooster van punten. De roosterpunten zijn in dit geval gepositioneerd op de massamiddelpunten van de polymeren. De aanpassing van de Navier-Stokes vergelijking bestaat hoofdzakelijk uit de toevoeging van een extra krachtterm, die de krachten tussen de polymeren onderling overdraagt naar de vloeistof. Deze methode is gebaseerd op het Galilei-invariante eerste orde Brownse Dynamica algoritme, ontwikkeld door Padding en Briels [J. Chem. Phys. 141, 244108 (2014)]. Deze koppeling maakt de stroming van de achtergrond vloeistof niet-Newtons. De koppeling is kan heel eenvoudig worden geïmplementeerd omdat de vloeistof blobs impliciet aanwezig zijn op dezelfde posities zoals de massamiddelpunten van de polymeren. We hebben deze methode toegepast om de stroming 
van de model-sterpolymeer oplossing tussen twee oneindige vaste platen te bestuderen. Passende grensvoorwaarden aan het vast-vloeistof interface zijn opgelegd met behulp van de methode van Morris et al. [J. Comp. Phys. 136, 214 (1997)].

Hoewel de methode beschreven in hoofdstuk 2 goede resultaten oplevert en rekenkundig efficiënt is, kent deze methode toch een aantal beperkingen. Het is namelijk niet mogelijk om de resolutie van de achtergrond vloeistof onafhankelijk te kiezen omdat deze per definitie dezelfde is als de concentratie van de polymeren. Ook is het niet mogelijk, zonder aanpassingen van het algoritme, een geschikte toestandsvergelijking voor de vloeistof te kiezen. Daarom hebben wij ons ten doel gesteld het model te verbeteren door twee verschillende soorten deeltjes te gebruiken, één voor de polymeren en één voor de vloeistof. De koppeling tussen deze twee is echter niet zo eenvoudig als met de vorige methode, omdat nu de vloeistof blobs niet meer aanwezig zijn op dezelfde plaats als de massamiddelpunten van de polymeren. Daartoe hebben wij we een nieuwe aanpak ontwikkeld, die we Hydrodynamically Coupled Brownian Dynamics (HCBD) noemen. Daarin zijn de bewegingen van de polymeren en de vloeistof, die nu door onafhankelijke blobs gerepresenteerd worden, gekoppeld door middel van een speciale interactie term die zo geconstrueerd is dat het lokale momentum onveranderd blijft.

Hoofdstuk 3 beschrijft de ontwikkeling van deze techniek en enkele eenvoudige toepassingen. Voor de toepassingen hebben wij een polymeer model gekozen waarin de conservatieve interacties tussen de polymeren beschreven worden met behulp van de Flory-Huggins vrije energie en geheugen in het systeem wordt geïntroduceerd door middel van de Responsive Particle Dynamics (RaPiD) methode. Met dit model hebben we de reologie van twee oplossingen met verschillende polymeer concentraties bestudeerd door hun response op een homogene afschuiving met behulp van de Lees-Edwards-methode, en op een ruimtelijk variërende afschuif-omgeving door middel de Reverse Poiseuille Flow techniek te simuleren. We hebben geconstateerd dat de polymeren zeer goed met de vloeistof zijn gekoppeld, wat blijkt uit de afwezigheid van een enig verschil tussen hun stroom snelheden. Verder hebben we het voor polymeer oplossingen kenmerkende verschijnsel van shear thinning en cross 
flow migration gevonden, welke experimenteel geobserveerd zijn.

In Hoofdstuk 4 is de door ons ontwikkelde nieuwe HCBD techniek gebruikt om de achtergrond vloeistof met een meer realistisch polymeermodel, namelijk RaPiD met Finitely Extensible Nonlinear Elastic (FENE) halters te koppelen. In dit polymeer model, worden de polymeren weergegeven als een eindig niet-lineaire elastische halter in plaats van een puntdeeltje zoals in RaPiD. Dit brengt, naast de externe, quasi elastische geheugen term van $\mathrm{RaPiD}$, intra-moleculaire elasticiteit in het systeem en stelt ons in staat om elastische instabiliteiten te bestuderen die wordt vertoond door polymeer oplossingen. We hebben de stroming van deze modelpolymeer oplossing voor twee verschillende concentraties in een homogeen afschuif veld gesimuleerd. Voor de hoogste concentratie hebben wij het sinds enkele tientallen jaren bekende shear banding fenomeen gevonden en de bijbehorende tijdontwikkeling bestudeerd. Bovendien hebben wij gevonden dat bij bepaalde afschuif snelheden de ontstane banden zelf instabiel worden en spontaan microstructuren zoals shear-rolls en streaks vormen.

In Hoofdstuk 5 is de stroming van de polymeer oplossing uit het vorige hoofdstuk door een systeem van periodiek geordende cilinders en balken bestudeerd. Deze structuren zijn zó gekozen dat zij verschillende configuraties, zoals die in poreuze media voor komen, nabootsen. In beide gevallen was de porositeit en de drukval over de media dezelfde. Bovendien, hebben we in beide gevallen twee verschillende, extreme stroomrichtingen gekozen. We hebben de verschillen in de opkomende stroompatronen en polymeerconcentraties in de twee verschillende media waargenomen. We zien interessante correlaties tussen de polymeerconcentraties en de stroomprofielen als een resultaat van cross-flow migratie van de polymeren. Dit benadrukt een voordeel van ons op grofkorrelige deeltjes-gebaseerde model in tegenstelling tot conventionele CFD-methoden, omdat onze methode ervoor zorgt dat polymeren inhomogeen over het toegankelijke volume kunnen worden verdeeld.

Kort samengevat, hebben we een nieuwe HCBD-techniek ontwikkeld, die ieder polymeermodel gebaseerd op Brownse Dynamica kan koppelen met een achtergrond vloeistof model gebaseerd op Smoothed Particle Hydrodynamics. Wij hebben laten 
zien dat ons model eenvoudig kan worden gebruikt om de stroming van polymeer oplossingen door poreuze media te bestuderen, wat één van de belangrijkste doelen van dit proefschrift was. Een belangrijk aspect van onze methode is dat zij niet beperkt is tot het bestuderen van de stroom van polymeeroplossingen, maar kan gemakkelijk worden uitgebreid om de stroom van visco-elastische oppervlakteactieve stoffen te bestuderen of meerfasige stroom door poreuze media om te begrijpen hoe polymeeroplossingen olie uit een oliereservoir verdringen. Dergelijke simulaties zijn van groot belang om verdringing-operaties met behulp van polymeer oplossingen in de Enhanced Oil Recovery beter te begrijpen. De hiermee geproduceerde extra olie kan als grondstof dienen in de petrochemische industrie of als brandstof dienen om zo bij te dragen tot de steeds toenemende vraag naar energie consumptie. Ons werk kan ook gebruikt worden om andere processen in de chemische industrie beter te begrijpen, zoals polymeer extrusie en food processing. Het is dus een nieuwe tool die kan worden gebruikt om het wetenschappelijk inzicht te bevorderen voor verschillende fenomenen van interesse gerelateerd aan een breed scala van niet-Newtoniaanse vloeistoffen met behulp van computersimulaties. 


\section{Scientific Output}

\subsection{Publications}

1. V. R. Ahuja, J. van der Gucht and W. J. Briels.

Coarse-grained simulations for flow of complex soft matter fluids in the bulk and in the presence of solid interfaces.

The Journal of Chemical Physics, 145(19), 194903 (2016).

https://dx.doi.org/10.1063/1.4967422

2. V. R. Ahuja, J. van der Gucht and W. J. Briels.

Hydrodynamically Coupled Brownian Dynamics: A coarse-grain particle-based Brownian dynamics technique with hydrodynamic interactions for modeling self-developing flow of polymer solutions.

The Journal of Chemical Physics, 148(3), 034902 (2018).

https://doi.org/10.1063/1.5006627

3. V. R. Ahuja, J. van der Gucht and W. J. Briels.

Hydrodynamically Coupled Brownian Dynamics simulations for studying rheology of complex soft matter systems.

In preparation.

4. V. R. Ahuja, J. van der Gucht and W. J. Briels.

Hydrodynamically Coupled Brownian Dynamics simulations of polymer solution flow through porous media.

In preparation.

\subsection{Talks}

1. V. R. Ahuja, J. van der Gucht and W. J. Briels.

Particle-based simulations of flowing visco-elastic fluids.

Burgersdag - Annual J.M.B.C. Fluid Mechanics Conference.

TU Delft, The Netherlands (2015) 
2. V. R. Ahuja, J. van der Gucht and W. J. Briels.

Particle-based simulations of flowing visco-elastic fluids.

SoftComp Annual Meeting.

Ancona, Italy (2015)

3. V. R. Ahuja, J. van der Gucht and W. J. Briels.

Particle-based simulations for flow of complex soft matter fluids.

Soft Matter+ Colloquium.

University of Twente, the Netherlands (2016)

\subsection{Posters}

1. V. R. Ahuja, J. van der Gucht and W. J. Briels.

Particle-based simulations of flowing visco-elastic fluids.

SUPOLEN Conference and Summer School.

Capri, Italy (2015)

2. V. R. Ahuja, J. van der Gucht and W. J. Briels.

Coarse-grained simulations for flow of soft matter systems with solid interfaces.

Physics of Soft and Biological Matter (IOP).

Cambridge, U.K. (2016)

3. V. R. Ahuja, J. van der Gucht and W. J. Briels.

Coarse-grained simulations for flow of highly frictional soft matter systems.

International Soft Matter Conference.

Grenoble, France (2016)

4. V. R. Ahuja, J. van der Gucht and W. J. Briels.

Coarse-grain simulations for flow of highly frictional soft matter systems.

Computational Sciences for Future Energy Conference.

Utrecht, the Netherlands (2016) 


\section{Social Impact}

Technological development has surely brought with it a lot of comfort in the lives of several people on this planet and has raised their standard of living. To truly understand the impact of this technological development, it will suffice to simply compare the lives of people in a very well developed country with those that live a country that is still under development. However, this technological development inevitably comes at a cost. The per-capita energy consumption of the developed countries is typically much higher than the developing countries. As the technological development spreads to more regions on this planet, the global energy demand is going to increase substantially. Furthermore, technology itself is constantly evolving and with more advanced technology typically comes a greater need for energy. It will suffice to look at the energy consumption of these so-called 'developed countries' over the period of last hundred years. Although these countries are being labelled as 'developed' because they are indeed developed compared to the other countries at the time, still there is constantly an improvement in technology even in these countries. Along with this development, the dreams of humanity get bigger and so does the required energy to fuel these dreams. In the $21^{\text {st }}$ century, there are talks of future missions to inhabit other planets and it is needless to say that the energy required for achieving such great feats is going to be humongous.

To meet the ever-increasing energy demand of humanity is certainly a challenge of our times. Technologies for harnessing unconventional sources of energy are constantly being developed. While there is a very encouraging increase in the proportion of global energy demand met with renewable energy sources, much of our energy needs are still met with fossil fuels. Although conventional fossil fuels are able to meet much of the demand of our times, it will certainly not be the case in the future as these resources are limited. Many oil reservoirs around the world are becoming 'mature' and the oil production of these wells is declining. It is however a known fact that although the oil wells are labeled 'mature', still almost half of the original oil in place in the reservoir is still not extracted. This is because recovering the additional oil that is trapped in the reservoir is a challenge in itself. Although various meth- 
ods of enhanced oil recovery have been developed over the years, still a fundamental understanding of the fluid flow within the complex reservoir is yet to be developed fully. This flow is even more complex in case of what are known as 'polymer flooding' operations, where polymer solutions are injected to recover additional oil from the reservoirs. This is because the addition of polymers to water makes the fluid viscoelastic and the flow of viscoelastic fluids through porous media is still a hot topic of research. The work that we have presented in this thesis tries to shed some light on how these polymer solutions flow through porous media. Particle based simulation techniques such as the one we have developed help bridge the understanding of the relation between macroscopic properties of these complex fluids and the microscopic interactions between the molecules present in these fluids. Although this complex problem is far from solved, this work provides the basis for further development in this area.

Besides the social impact of the research work that I have done during my Ph.D. studies, I would also like to shed some light on what I could do on a personal level thanks to this opportunity given to me for working here. I count myself amongst some of the most fortunate people on this planet. I come from a middle-class family in India, which entails that success in life is not guaranteed to us but we must work hard for it. My grandparents and parents have worked very hard over the years to provide our family a good standard of living. They made it possible for me to obtain an education of the highest possible standards in India, which has provided a strong foundation for my career. Having said that, I also realize that there still exist plenty of other hard-working people in India who haven't got what they truly deserve despite working very hard. Unfortunately, some of the worst affected people in our country actually tend to be farmers, the very people who feed us by growing grains and vegetables thereby making our country largely self-reliant in terms of food despite the large population.

It is therefore my social responsibility to give back to the poor farmers of India what they truly deserve. Fortunately, there exist many organizations who help people like me achieve that goal. One such organization that I found by doing some research 
on the internet is Milaap. It's a crowdfunding organization that collects money from voluntary donors and interfaces with several NGO's that effect change at the groundlevel. I am very grateful to them for providing this service for a nominal fee. It is heartening to see the amount of donations pouring in from around the world every minute, which shows there exist so many generous people in the world. As this organization primarily interfaces with Indian NGO's, naturally many of the donors are Indians but I must say there are also a few regular donors that I see on the website that have clearly foreign names like Gijsbert for example, who might actually be Dutch, guessing from his name. I am very grateful to all the people around the world helping in these social causes and helping us to improve the situation in our country. Over the years, I have also done a little bit to help these organizations by providing several loans to these poor village-dwelling farmers from all over the country through this crowdfunding organization called Milaap. I hereby urge other fortunate people too to also provide whatever help they can in this endeavor. Although your individual contribution may be small, but we can surely make a difference together. As they say in Dutch: 'Geloof in de kracht van samen! Samen kunnen wij een verschil maken!'

As you can see from the info-graphic shown in fig.9.1, the cause that I have provided the most loans for is to enable poor villagers to buy solar lanterns. Many of these farmer don't have a stable electricity supply. My hope is that this will provide them a way to be more productive at home even after dark and probably help some kids learn and do their homeworks in the evening, who one day perhaps become engineers and doctors and give back to the society again. It is also perhaps fitting to lend my money to providing solar energy given that my project is actually about 'Computational Science for Future Energy'. Moreover, as you can see from the infographic, the poor farmers almost always repay their loan in full, which enables me to recycle the money to lend it to even more farmers, thereby perpetually continuing the cycle and helping more and more people with the same money. I think this is the best investment I have ever made and I shall keep investing and hope you do too for a better world. Thus, I am very thankful to have got this wonderful opportunity to work here in this beautiful country called the Netherlands which has enabled me to 
produce some scientific as well as social output during the four and a half years that I lived here.

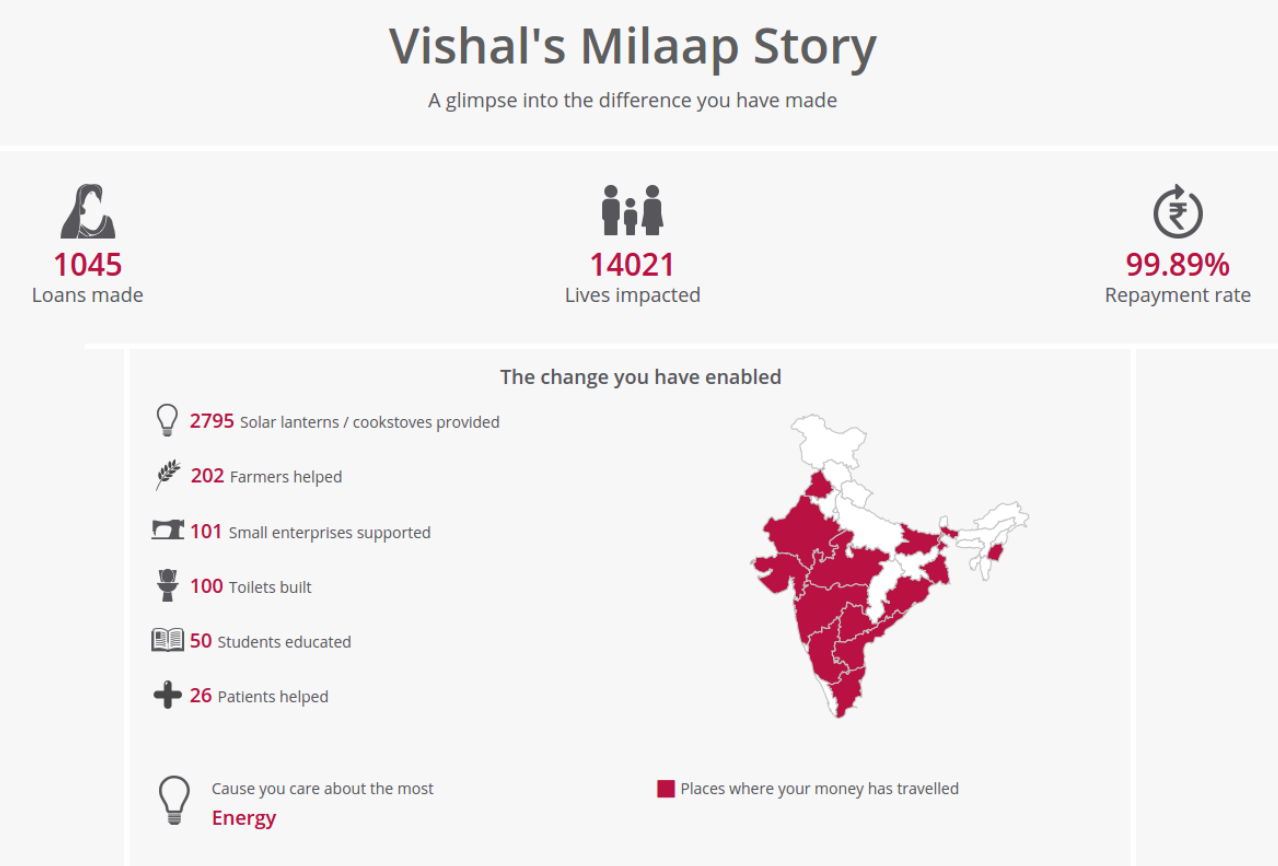

Figure 9.1: An info-graphic showing the social causes that I have lent my money for and the places in India my money has travelled to through the NGO's that I have supported through the crowdfunding organization Milaap.

Finally, I would like to make a small correction to the above info-graphic - It's not really just 'Vishal's Milaap Story' but rather the 'Story of all of us who are trying to make a difference'. It's not me who has made this small difference, rather its all of you who have made this difference through me. Thanks everyone! Ending this section with these lines written by a great Indian poet Mirza Ghalib (1797 - 1869):

Original lines: Hazaaron khaavihishein aiseen ki har khaavahish pe dam nikle... Bahut nikle mere armaan, lekin fir bhi kam nikle!

Translation: Thousands of such ambitions, each ambition worth dedicating my whole life to...Many such ambitions of mine have materialized, yet not nearly enough! 


\section{Acknowledgments}

First and foremost, I would like to thank my parents and grandparents (my first gurus) from the bottom of my heart as they really are the stable pillars that have supported the edifice of my life so far. I truly am immensely grateful to them for providing me with a wonderful upbringing. It is the moral values that I learnt from them and the formal education that I got access to because of them, that have served together as a strong foundation for my scientific career. I am also thankful to my relatives and the very first friends I made in my life in the wonderful city of Mumbai, particularly Harsh, without whom my childhood would not have been so much fun. I am very grateful to all my teachers from high school, college and the universities that I have attended in my life. I have been very fortunate to have got the opportunity to study at such remarkable institutions of excellence as Institute of Chemical Technology - Mumbai (formerly UDCT) and Indian Institute of Technology Bombay (IIT Bombay), which provided me with the possibility to apply for a Ph.D. position in another center of excellence - University of Twente, where I have conducted my Ph.D. research. It is here in the University of Twente, that I got the opportunity to work with a very experienced, knowledgeable and witty professor - Prof. Wim Briels, without whose inputs and directions the work presented in this thesis would have been impossible. I would also like to thank Prof. Jasper van der Gucht from Wageningen University for his suggestions and guidance, which certainly helped me improve the quality of my research work.

I would like to thank all the members of the Computational Chemical Physics (CCP) group including the former members of the CBP and the BES groups, the two sister-groups which later merged into the CCP group as we know it today. The first thing I noticed when I joined the group in 2013 was how international it was. Besides Wim and Wouter, everyone was from abroad - Igor from Brazil, Li from China, Gabriel from Colombia, Barry from Ireland, Claudia, Matteo and Riccardo from Italy, Csaba and Ioana from Romania, and the two new students were me and Habib from India and Indonesia respectively. Two more Indian students would later join the group in the years to come - Vishal and Monika. It's a pleasure knowing each 
and every one of them. I always found it so amazing how this group brought together people from different cultures around the globe, thereby providing a platform for really interesting exchange of different ideas and perspectives. I certainly benefited a lot from the very diverse perspectives that I got to understand while discussing with fellow group members over coffee breaks. I also want to thank Els (the present secretary for CCP), Carin (the former secretary), Mara (the secretary in Wageningen), Maria (from FOM) and the Shell personnel for handling all the administrative work so efficiently. More details about my interaction with each of the group members follow in the next two paragraphs.

In the initial months that I was here, Igor and Gabriel helped me understand the $\mathrm{RaPiD}$ code, for which I am very grateful. Li was my office-mate for about a couple of years, during which we developed a good camaraderie. Barry always kept us entertained, constantly blurring the line between fact and fiction with his funny 'anecdotes'. Ioana has always been very helpful - right from the time I was corresponding with her from India before coming to the Netherlands asking her about the accommodation options in Enschede, until the time that she helped me submit my first paper. I am very grateful for all her help. Matteo has been someone who was like a brother to me when I came to the Netherlands - someone who made me feel comfortable and at home in a place which was very new to me. Its always a bit difficult to adjust to a new place with a different culture. Although I didn't feel a big culture-shock as I came from a pretty modern city in India, yet you just don't feel at home. You need someone you can talk to in a free manner without constantly being judged and Matteo was exactly that. We spoke about so many different things - politics, culture, gaming, etc. and it was always a pleasure speaking with him. Csaba was a funny character I met in the Netherlands, whom I will most remember for all the debates we had during our coffee breaks over the years. Riccardo was there too during our coffee breaks and I got the feeling that he rather enjoyed seeing the two of us fight while he ate his popcorn (I mean drank his coffee). Matteo was there too during these breaks of course and much like the other Italian enjoyed watching us fight. Oh but he wouldn't stop at that of course. If there was ever a peaceful coffee break, rest assured 
that Matteo would come up with a weird question like "What is the body temperature of a cow?" just to spark a random debate between me and Csaba that we would have never had otherwise. But then again with Csaba, you never know. He could bring up the wierdest topics starting with the sentence "There is a hot debate on the internet about" and the other half of the sentence could be as weird as "whether you would rather fight 50 duck-sized horses or a horse-sized duck?"

Wouter helped me with quite a few tips and tricks related to Linux, Fortran, etc. and I am very thankful for that. Claudia was someone I would seek advice from in the absence of Wim and she was always very helpful. Besides, she was sometimes like a motherly figure who would worry that we (the CCP students) might catch a cold because we were not wearing a warmer sweater. Wim was really a father figure to me in the Netherlands - both in the scientific sense and also personally. I have learnt a lot from him over the four and half years of collaboration with him. He introduced me to the wonderful world of polymers performing Brownian Dynamics, and I coupled these polymers with the background fluid and gave him Hydrodynamically Coupled Brownian Dynamics as 'gurudakshina' (a Sanskrit term which means a gift that the pupil must give to the guru at the end of his training as a token of gratitude). Well, to be more precise, it isn't really fair to call it entirely my gift to him really but rather it is something that we came up with together (gifted ourselves with) as a result of a beautiful collaboration, where he had the conceptual idea of what must come out in the end, and I brought it to fruition by intuitively constructing an interaction term that would do the job. When it worked, I remember we were very happy to see the results and had a nice celebration when the paper we wrote about it was accepted. Besides sharing his scientific and technical know-how, Wim also shared his knowledge of the Dutch language with me. Although I pick up sounds from other languages quickly thanks to my knowledge of 6 other languages that I speak (for which I must thank my parents, grandparents and friends but particularly my mom) which cover a lot of sounds, yet I used to find it difficult to pronounce the diphthong 'ui' in Dutch and he really taught me how to say it properly. This is very similar to the story that my mom once told me about how she put in a lot of effort when I was a kid to get me to 
pronounce the 'r', which I apparently found very difficult back then. I have to also thank him for improving my usage of words in the English language. Finally, I wish to once and for all end the controversial tale that Wim tells about me by telling the full sentence that I once told Wim and life has never been the same since. After reading my first paper, he told me that I know a lot of words in English, some of which were not even known to him, to which I responded with "Although I might know a few more English words than you because my language of instruction since school has been English, yet I must say your usage of the words is much better than mine." Of course, he never 'manages to recollect' the second half of the sentence whenever he tells this story.

When I first arrived in the Netherlands, I lived in the ITC hotel in Enschede for the first 6 months, where I made a lot of good friends - Raja, Jothi, Riddhi, Siddhi, Sneha, Somi, Kunal, Harish, Devashree, Akshay, Mitava, Ana and many others. I also became friends with Devashish, Vishal Agarwal and Manas and we really had some great times. All the delicious Indian dishes which we prepared together on weekends really gave me the feeling of being at home. I also made a lot of friends at the Thibats table tennis club of University of Twente such as Wesley, Chantal, Ina, Linda, Martine, Emilio, Arturo, Fang, Celestine and many more. I'm grateful to Thibats not just for their training and the friends I made but also because it is thanks to them that I won the only trophy that I have ever won in my life for any sport whatsoever. It motivated me so much that I kept playing for another year and in the next tournament my team won the championship of the Enschede region at our level. I also met Sahana through the club, where she came to play table-tennis once. I remember that we went to the sports canteen right after the training session which is when we had our first conversation and she never came back to play again. Hahaha...true story!

Sahana and I became very good friends over the years and shared some of our best moments in the Netherlands together. Kriti was another very good friend of mine in the Netherlands. They were always there for me - both in sickness and during happy times. I remember having spent some very memorable and musical evenings 
with Sahana, Kriti and Vishal Metri at Sahana's place. The wonderful thing about Sahana's place was that you could get truly immersed in music - not because of the acoustics of her apartment, but because there really was nothing there that could distract you. There was no internet, hardly any cell phone coverage and mostly not even enough light! Jokes apart, I really cherish the times that we spent together and the Netherlands really became a home away from home thanks to you all.

I must say I really learnt a lot from all the friends that I made in the Netherlands over the years. Particularly from Vishal Metri (a very co-operative office-mate and an equally good friend), I learnt a lot of stuff on a wide variety of subjects. I really mean a wide range of subjects - spanning from electrical circuits, computer hardware architecture, movies, books, music, spirituality - Advaita Vedanta, Buddhism...you name it. The breadth of his knowledge is simply amazing to me as he seems to know something about practically everything. He enlightened me a lot with his knowledge on these diverse subjects and he also recommended quite a few good movies and books too for which I'm very grateful. I also made a Syrian friend - Issa, who is absolutely the best hair stylist I have ever known and I highly recommend the 'Unique Gentleman Herenkapper' in Hengelo. He is not just great at his work but is also a very kind and nice person to chat with. It was a real pleasure seeing him every time I made an appointment for a haircut. He would give me a warm welcome and we would have a great conversation every time.

I would also like to acknowledge Pramodji and his family for inviting me to their house for Ganesh Chaturthi in the very first year that I came here and it is through them that I became friends with many people of the Aadhaar community, who do a brilliant selfless job and hats off to them for that. I also acknowledge all the members of the Indian Student Association for organizing such wonderful events like Holi and Diwali every year. I would also like to acknowledge Mrs. and Mr. Hofstede, in whose house I stayed for four years as a tenant while I lived in Hengelo. They were really very nice to me (hele aardige mensen as they say in Dutch). They would greet me very warmly every time we met and I had some very nice conversations with them every once in a while. I spoke to them in Dutch mostly and that gave me a good 
regular practice I must say. I would also like to acknowledge Catherine, Margreet, Lucia, Joana, Lawrence, Effie, Andrea, Bojan, Anja, Elahe, Shantanu, Poorvi, Vamsi, Naveen, Omkar, Anirban (Dada), Himanshu (Netaji) who are certainly some of the nicest and kindest people I met in Europe. Of all these people, I must thank Catherine expressly for her deep affection, constant support and help with my work too particularly in pointing out typos and mistakes in my first paper. I would also like to thank Margreet, whom I met only towards the end of my time here in the Netherlands but we did some fun things together like the Kerstfeest on Tweede Kerstdag, New Year celebrations, etc. I'm also thankful to her for proof-reading the samenvatting in my thesis and consoling me that it was not bad in the first place despite the fact that practically the whole page was red in her corrected version!

There are also many other people whom I may not have mentioned by name but it certainly does not mean that their contribution to my life was any less important. Overall, my stay in the Netherlands has been awesome and so I am very happy with the decision I made four and a half years ago to come to the Netherlands - the land of tall people living in short houses, having high income but low spending. Jokes apart, it really is a very nice country to live in - very systematic planning, great infrastructure, awesome museums, very liberal people and of course lot of the three W's - windmills (makes sense in a flat-land with unobstructed wind), wooden shoes (most pointless thing I've seen in the Netherlands) and water (I'm thinking of all the dikes). The constant pumping of water to keep their 'lower lands' dry has certainly made the Dutch - the Royal Plumbers of the World or should I say Koninklijke Loodgieters van de Wereld! They should make an advertisement: Suffering from all the water running into your streets after a hurricane or a massive flood? Don't worry. The Dutch have you covered. Daar zijn we trots op!

Overall, I had a great time in the Netherlands and I shall never forget the wonderful years of my life that I spent there. As a token of gratitude, I would like to end my thesis in Dutch - Ik had een gezellige tijd in Nederland. Hartelijk bedankt allemaal! 
This thesis deals with model development for particle-based flow simulations of non-Newtonian fluids such as polymer solutions. A novel computational technique called Hydrodynamically Coupled Brownian Dynamics (HCBD) is presented in this thesis. This technique essentially couples the Brownian motion of the polymers to the flow of the solvent in a way that momentum is locally conserved. Potential applications include polymer flooding operations in Enhanced Oil Recovery (EOR), polymer processing, food processing etc.

ISBN $\quad 978-90-365-4562-4$

DOI $\quad 10.3990 / 1.9789036545624$

URL $\quad$ https://doi.org/10.3990/1.9789036545624
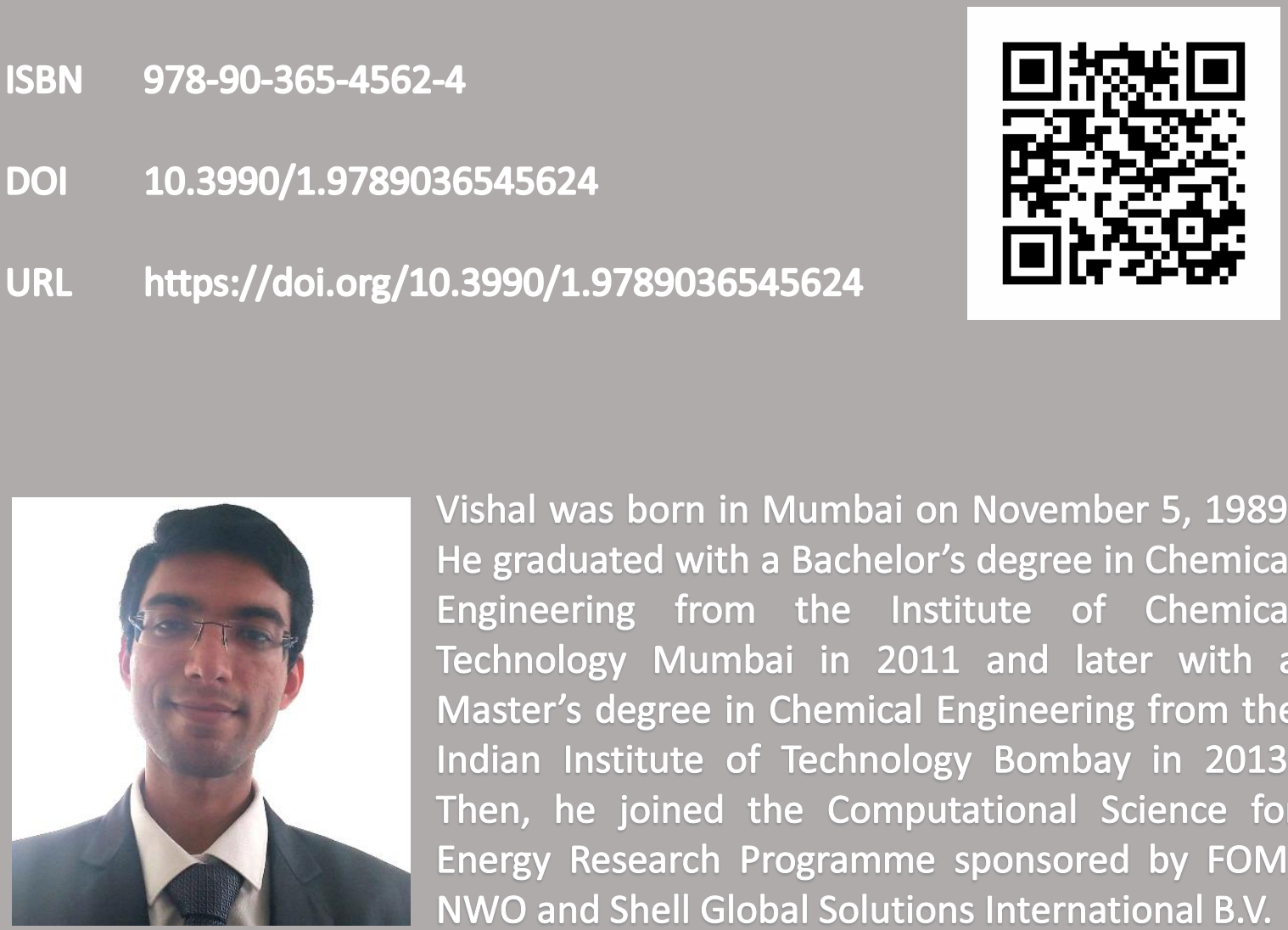

Vishal was born in Mumbai on November 5, 1989. He graduated with a Bachelor's degree in Chemical Engineering from the Institute of Chemical Technology Mumbai in 2011 and later with a Master's degree in Chemical Engineering from the Indian Institute of Technology Bombay in 2013. Then, he joined the Computational Science for Energy Research Programme sponsored by FOM, NWO and Shell Global Solutions International B.V.

He undertook his Ph.D. research at the Computational Chemical Physics group of the University of Twente in collaboration with the Physical Chemistry and Soft Matter group of Wageningen University. He now works as a Computational Researcher at Shell Technology Centre Bangalore in India since April 2018. 